\title{
REPRESENTATION ZETA FUNCTIONS OF COMPACT $p$-ADIC ANALYTIC GROUPS AND ARITHMETIC GROUPS
}

\author{
NIR AVNI, BENJAMIN KLOPSCH, URI ONN, AND CHRISTOPHER VOLL
}

\begin{abstract}
We introduce new methods from p-adic integration into the study of representation zeta functions associated to compact $p$-adic analytic groups and arithmetic groups. They allow us to establish that the representation zeta functions of generic members of families of $p$-adic analytic pro- $p$ groups obtained from a global, 'perfect' Lie lattice satisfy functional equations. In the case of 'semisimple' compact $p$-adic analytic groups, we exhibit a link between the relevant $\mathfrak{p}$-adic integrals and a natural filtration of the locus of irregular elements in the associated semisimple Lie algebra, defined by centraliser dimension.

Based on this algebro-geometric description, we compute explicit formulae for the representation zeta functions of principal congruence subgroups of the groups $\operatorname{SL}_{3}(\mathfrak{o})$, where $\mathfrak{o}$ is a compact discrete valuation ring of characteristic 0 , and of the groups $\mathrm{SU}_{3}(\mathfrak{O}, \mathfrak{o})$, where $\mathfrak{O}$ is an unramified quadratic extension of $\mathfrak{o}$. These formulae, combined with approximative Clifford theory, allow us to determine the abscissae of convergence of representation zeta functions associated to arithmetic subgroups of algebraic groups of type $A_{2}$. Assuming a conjecture of Serre on the Congruence Subgroup Problem, we thereby prove a conjecture of Larsen and Lubotzky on lattices in higher-rank semisimple groups for algebraic groups of type $A_{2}$ defined over number fields.
\end{abstract}

\section{Contents}

1. Introduction

Part 1. p-Adic formalism

\section{Part 2. Applications}

6. Formulae for principal congruence subgroups of $\mathrm{SL}_{3}(\mathfrak{o})$ and $\mathrm{SU}_{3}(\mathfrak{O}, \mathfrak{o})$

7. Abscissae of convergence for arithmetic groups of type $A_{2}$

\section{Appendices}

Appendix A. Algebraic groups of type $A_{2}$

Appendix B. Adjoint action of $\mathrm{GL}_{3}\left(\mathbb{F}_{q}\right)$ on $\mathfrak{s l}_{3}\left(\mathbb{F}_{q}\right)$

Appendix C. Adjoint action of $\mathrm{GU}_{3}\left(\mathbb{F}_{q^{2}}, \mathbb{F}_{q}\right)$ on $\mathfrak{s u}_{3}\left(\mathbb{F}_{q^{2}}, \mathbb{F}_{q}\right)$

References

2000 Mathematics Subject Classification. 22E50, 22E55, 20F69, 22E40, 11M41, 20C15, $20 \mathrm{G} 25$.

Key words and phrases. Representation growth, $p$-adic analytic group, arithmetic group, Igusa local zeta function, $\mathfrak{p}$-adic integration, Kirillov orbit method.

Avni was supported by NSF grant DMS-0901638. 


\section{INTRODUCTION}

1.1. Background and motivation. Let $G$ be a group and denote, for $n \in \mathbb{N}$, by $r_{n}(G)$ the number of isomorphism classes of $n$-dimensional irreducible complex representations of $G$; if $G$ is a topological or an algebraic group, it is tacitly understood that representations are continuous or rational, respectively. We assume henceforth that $G$ is (representation) rigid, i.e. that $r_{n}(G)$ is finite for all $n \in \mathbb{N}$.

In finite group theory there is a fruitful tradition in studying character degrees and conjugacy classes; e.g. see [28, 42] and references therein. Interesting new phenomena occur when one takes an asymptotic point of view, as illustrated in [37. which focuses on character degrees of finite groups $H$ of Lie type as $|H|$ tends to infinity. Likewise, in the subject of representation growth one studies, for an infinite group $G$, the arithmetic properties of the sequence $r_{n}(G), n \in \mathbb{N}$, and its asymptotic behaviour as $n$ tends to infinity. This line of investigation draws its inspiration also from the area of subgroup growth which, in a similar vein, is concerned with the distribution of finite index subgroups in $G$; e.g. see [39, 16].

The group $G$ is said to have polynomial representation growth (PRG) if the sequence $R_{N}(G):=\sum_{n=1}^{N} r_{n}(G), N \in \mathbb{N}$, is bounded by a polynomial in $N$. An important tool to study the representation growth of a PRG group $G$ is its representation zeta function, viz. the Dirichlet series

$$
\zeta_{G}(s):=\sum_{n=1}^{\infty} r_{n}(G) n^{-s}
$$

where $s$ is a complex variable. It is well-known that the abscissa of convergence $\alpha(G)$ of the series $\zeta_{G}(s)$, i.e. the infimum of all $\alpha \in \mathbb{R}$ such that $\zeta_{G}(s)$ converges on the complex right half-plane $\{s \in \mathbb{C} \mid \operatorname{Re}(s)>\alpha\}$, gives the precise degree of polynomial growth: $\alpha(G)$ is the smallest value such that $R_{N}(G)=O\left(1+N^{\alpha(G)+\varepsilon}\right)$ for every $\varepsilon \in \mathbb{R}_{>0}$.

Key advances in describing the representation growth of arithmetic groups were made by Larsen and Lubotzky in [35]. At present our general understanding of the representation theory of 'semisimple' arithmetic groups, like $\mathrm{SL}_{n}(\mathbb{Z})$, rests on three major theories, namely Margulis super-rigidity, the representation theory of simple algebraic groups and Deligne-Lusztig theory for finite groups of Lie type. What is missing is a picture of the representations of groups of Lie type over finite local rings; however, the corresponding classification problem is considered to be 'wild'. Focusing on degrees of characters and taking an enumerative point of view, we make in this paper significant progress in describing the representation growth of arithmetic groups and compact $p$-adic analytic groups which arise as their completions. Connections between our work and number theory manifest themselves in classical papers of Kloosterman [33] as well as in recent developments related to the Langlands programme. For instance, certain representations of groups of Lie type over compact discrete valuation rings play a central role in the construction of super cuspidal representations of reductive groups over local fields within the framework of the local Langlands correspondence; cf. [9].

More concretely, we study the representation zeta functions of two types of groups: compact $p$-adic analytic groups and arithmetic groups. Before we describe our main techniques and results in Section 1.2, we highlight some features of the representation growth of these two types, and explain how they are interwoven in the theory of representation zeta functions.

A compact $p$-adic analytic group $G$ is rigid if and only if it is FAb, i.e. if every open subgroup of $G$ has finite abelianisation; cf. [5, Proposition 2]. In [31, Jaikin-Zapirain 
proved rationality results for the representation zeta functions of FAb compact $p$-adic analytic groups using tools from model theory. In particular, the representation zeta function of a FAb compact $p$-adic analytic pro- $p$ group is rational in $p^{-s}$. (In the special case $p=2$, the assertion is currently known to be true for uniformly powerful groups, but conjectured to hold generally, as for odd $p$.) Key examples of FAb compact $p$-adic analytic groups are the special linear groups $\mathrm{SL}_{n}(\mathfrak{o})$ and their principal congruence subgroups $\mathrm{SL}_{n}^{m}(\mathfrak{o})$, where $\mathfrak{o}$ is a compact discrete valuation ring of characteristic 0 and residue field characteristic $p$.

The arithmetic groups we are interested in are arithmetic subgroups of semisimple algebraic groups defined over number fields. More precisely, we consider groups $\Gamma$ which are commensurable to $\mathbf{G}\left(\mathcal{O}_{S}\right)$, where $\mathbf{G}$ is a connected, simply connected semisimple algebraic group, defined over a number field $k$ and $\mathcal{O}_{S}$ is the ring of $S$-integers in $k$, for a finite set $S$ of places of $k$ including all the archimedean ones. Let $\Gamma$ be of this form. Lubotzky and Martin showed that $\Gamma$ has PRG if and only if $\Gamma$ has the Congruence Subgroup Property (CSP); cf. [38. Suppose that $\Gamma$ possesses these properties. Then according to a result of Larsen and Lubotzky ([35, Proposition 1.3]) the representation zeta function of $\Gamma$ admits an Euler product decomposition. Indeed, if $\Gamma=\mathbf{G}\left(\mathcal{O}_{S}\right)$ and if the congruence kernel of $\Gamma$ is trivial, this decomposition takes the form

$$
\zeta_{\Gamma}(s)=\zeta_{\mathbf{G}(\mathbb{C})}(s)^{|k: \mathbb{Q}|} \cdot \prod_{v \notin S} \zeta_{\mathbf{G}\left(\mathcal{O}_{v}\right)}(s),
$$

where the product extends over all places $v$ of $k$ which are not in $S$. Here each archimedean factor $\zeta_{\mathbf{G}(\mathbb{C})}(s)$ enumerates the finite dimensional, irreducible rational representations of the algebraic group $\mathbf{G}(\mathbb{C})$; their contribution to the Euler product reflects Margulis super-rigidity. By $\mathcal{O}_{v}$ we denote the ring of integers in the completion $k_{v}$ of $k$ at the non-archimedean place $v$. The Euler product over the factors $\zeta_{\mathbf{G}\left(\mathcal{O}_{v}\right)}(s), v \notin S$, captures the representations of $\Gamma$ with finite image. The groups $\mathbf{G}\left(\mathcal{O}_{v}\right)$ are FAb compact $p$-adic analytic groups. An important family of examples of arithmetic groups with the CSP are the special linear groups $\mathrm{SL}_{n}(\mathbb{Z}), n \geq 3$, for which (1.1) reads

$$
\zeta_{\mathrm{SL}_{n}(\mathbb{Z})}(s)=\zeta_{\mathrm{SL}_{n}(\mathbb{C})}(s) \cdot \prod_{p \text { prime }} \zeta_{\mathrm{SL}_{n}\left(\mathbb{Z}_{p}\right)}(s) .
$$

Several of the results of [35] concern the abscissae of convergence of the local representation zeta functions occurring as Euler factors on the right hand side of (1.1) for suitable arithmetic groups $\Gamma$. Of particular interest is the dependence of these abscissae on natural invariants, such as the Lie rank of the ambient group of $\Gamma$ or the place $v$ at which $\Gamma$ is localised. With regards to abscissae of convergence of the global representation zeta functions for arithmetic groups, Avni proved in [1] that, for an arithmetic group $\Gamma$ with the CSP, the abscissa of convergence of $\zeta_{\Gamma}(s)$ is always a rational number. Larsen and Lubotzky made the following conjecture, which can be regarded as a refinement of Serre's conjecture on the Congruence Subgroup Problem.

Conjecture (Larsen and Lubotzky [35, Conjecture 1.5]). Let $H$ be a higher-rank semisimple group. Then, for any two irreducible lattices $\Gamma_{1}$ and $\Gamma_{2}$ in $H, \alpha\left(\Gamma_{1}\right)=\alpha\left(\Gamma_{2}\right)$.

In the current paper we introduce novel methods into the study of representation zeta functions of compact $p$-adic analytic groups and arithmetic groups, and use them to prove both local and global results. The main theorems of the current paper and related results from [3, 4] were announced in [2]. 
1.2. Main results. Our first two main results concern families of $p$-adic analytic pro- $p$ groups which arise, via $p$-adic Lie theory, from the completions of a global Lie lattice $\Lambda$, defined over the ring of integers of a number field. Examples of specific interest are families formed by the principal congruence subgroups of the compact $p$-adic analytic groups $\mathbf{G}\left(\mathcal{O}_{v}\right)$ featuring in (1.1). More generally, let $\mathcal{O}$ be the ring of integers of a number field $k$, and $\Lambda$ an $\mathcal{O}$-Lie lattice such that $k \otimes_{\mathcal{O}} \Lambda$ is a finite dimensional, perfect $k$-Lie algebra. Let $\mathfrak{o}=\mathcal{O}_{v}$ be the ring of integers of the completion $k_{v}$ of $k$ at a nonarchimedean place $v$ above the rational prime $p$. Given a finite extension $\mathfrak{O} \mid \mathfrak{o}$ of compact discrete valuation rings of characteristic 0 , let $\mathfrak{P}$ denote the maximal ideal of $\mathfrak{O}$ and write $f(\mathfrak{O}, \mathfrak{o})$ for the degree of inertia. Consider the $\mathfrak{O}$-Lie lattice $\mathfrak{g}(\mathfrak{O}):=\mathfrak{O} \otimes_{\mathcal{O}} \Lambda$. For all sufficiently large $m \in \mathbb{N}_{0}$, the principal congruence sublattice $\mathfrak{g}^{m}(\mathfrak{O}):=\mathfrak{P}^{m} \mathfrak{g}(\mathfrak{O})$ is potent and saturable; consequently it corresponds, via $p$-adic Lie theory, to a potent and saturable FAb pro- $p$ group $G^{m}(\mathfrak{O}):=\exp \left(\mathfrak{g}^{m}(\mathfrak{O})\right)$; see Section 2.1. We call such $m$ permissible for $\mathfrak{g}(\mathfrak{O})$. Proposition 2.3 states that, uniformly for any choice of the lattice $\Lambda$, all natural numbers $m$ exceeding an explicit linear bound in terms of the absolute ramification index $e\left(\mathfrak{O}, \mathbb{Z}_{p}\right)$ are permissible for $\mathfrak{g}(\mathfrak{O})$. In particular, if $\mathfrak{O}$ is unramified over $\mathbb{Z}_{p}$ and $p$ odd, then every $m \geq 1$ is permissible for $\mathfrak{g}(\mathfrak{O})$.

Our first theorem provides a universal formula for the zeta functions of the groups $\mathrm{G}^{m}(\mathfrak{O})$ and establishes the existence of local functional equations.

Theorem A. In the setup described above, there exist a finite set $S$ of places of $k$, $r \in \mathbb{N}$ and a rational function $R\left(X_{1}, \ldots, X_{r}, Y\right) \in \mathbb{Q}\left(X_{1}, \ldots, X_{r}, Y\right)$ such that, for every non-archimedean place $v$ of $k$ with $v \notin S$, the following is true.

There exist algebraic integers $\lambda_{1}=\lambda_{1}(v), \ldots, \lambda_{r}=\lambda_{r}(v)$, such that, for all finite extensions $\mathfrak{O}$ of $\mathfrak{o}=\mathcal{O}_{v}$ and for all $m \in \mathbb{N}_{0}$ which are permissible for $\mathfrak{g}(\mathfrak{O})$ one has

$$
\zeta_{\mathrm{G}^{m}(\mathfrak{D})}(s)=q_{v}^{f d m} R\left(\lambda_{1}^{f}, \ldots, \lambda_{r}^{f}, q_{v}^{-f s}\right),
$$

where $q_{v}$ denotes the residue field cardinality of $\mathfrak{o}, f=f(\mathfrak{O}, \mathfrak{o})$ and $d=\operatorname{rk}_{\mathfrak{O}}(\mathfrak{g}(\mathfrak{O}))=$ $\operatorname{dim}_{k}\left(k \otimes_{\mathcal{O}} \Lambda\right)$. Furthermore, the functional equation

$$
\left.\zeta_{\mathrm{G}^{m}(\mathfrak{O})}(s)\right|_{\substack{q_{v} \rightarrow q_{v}^{-1} \\ \lambda_{j} \rightarrow \lambda_{j}^{-1}}}=q_{v}^{f d(1-2 m)} \zeta_{\mathrm{G}^{m}(\mathfrak{O})}(s)
$$

holds.

Our second theorem concerns the possible poles of the zeta functions $\zeta_{\mathrm{G}^{m}(\mathfrak{O})}(s)$. It also provides information about how the abscissae of convergence of these zeta functions may change with extensions $\mathfrak{O}$ of the base ring $\mathfrak{o}$.

Theorem B. In the setup described above, there exists a finite set $P \subset \mathbb{Q}_{>0}$ such that the following is true.

(1) For all non-archimedean places $v$ of $k$, for all finite extensions $\mathfrak{O}$ of $\mathfrak{o}=\mathcal{O}_{v}$ and all $m \in \mathbb{N}_{0}$ which are permissible for $\mathfrak{g}(\mathfrak{O})$ one has

$$
\left\{\operatorname{Re}(z) \mid z \in \mathbb{C} \text { a pole of } \zeta_{\mathrm{G}^{m}(\mathfrak{D})}(s)\right\} \subseteq P .
$$

In particular, one has $\alpha\left(\mathrm{G}^{m}(\mathfrak{O})\right) \leq \max P$.

(2) There exists a set $V$ of non-archimedean places of $k$ of positive Dirichlet density with the property that for all $v \in V$, all finite extensions $\mathfrak{O}$ of $\mathfrak{o}=\mathcal{O}_{v}$ and all $m \in \mathbb{N}_{0}$ which are permissible for $\mathfrak{g}(\mathfrak{O})$ one has

$$
\alpha\left(\mathrm{G}^{m}(\mathfrak{O})\right)=\max P .
$$


Furthermore, if $v$ is any non-archimedean place of $k$ and if $\mathcal{O}_{v}=\mathfrak{o} \subseteq \mathfrak{O}_{1} \subseteq \mathfrak{O}_{2}$ is a tower of finite ring extensions, then for every $m \in \mathbb{N}_{0}$ which is permissible for $\mathfrak{g}\left(\mathfrak{O}_{1}\right)$ and $\mathfrak{g}\left(\mathfrak{O}_{2}\right)$ one has

$$
\alpha\left(\mathrm{G}^{m}\left(\mathfrak{O}_{1}\right)\right) \leq \alpha\left(\mathrm{G}^{m}\left(\mathfrak{O}_{2}\right)\right) .
$$

Remark 1.1. The proofs of Theorems $\mathrm{A}$ and $\mathrm{B}$ involve a description of the respective zeta functions in terms of $\mathfrak{p}$-adic integrals generalising Igusa local zeta functions. The study of the latter relies on deep algebro-geometric techniques and results; cf. [12]. These include resolutions of singularities in characteristic 0 and aspects of the Weil conjectures.

The rational function $R\left(X_{1}, \ldots, X_{r}, Y\right)$ in Theorem $\mathrm{A}$ arises from explicit formulae which admit further investigation; it is remarkable that there is no dependency on ramification. The main thrust of Theorem $\mathrm{B}$ is the following. Whilst the formulae established in Theorem A genuinely require finitely many exceptions, we show that the finite set of (real parts of) 'candidate poles' arising from the analysis of the generic places, viz. those with 'good reduction' modulo $\mathfrak{p}$, yields also a superset of the (real parts of) poles at the finitely many exceptional places which have 'bad reduction' modulo $\mathfrak{p}$.

Theorem Bimplies statements about the abscissae of convergence of the zeta functions of the $p$-adic analytic groups $\mathbf{G}\left(\mathcal{O}_{v}\right)$ which occur as non-archimedean factors in Euler products like (1.1). Indeed, for sufficiently large $m$, Theorem B applies to the principal congruence subgroups $\mathbf{G}^{m}\left(\mathcal{O}_{v}\right)$, and the abscissae of convergence of the zeta functions of commensurable groups coincide; cf. [35, Corollary 4.5].

Theorem B also has immediate applications to Larsen and Lubotzky's conjecture given at the end of Section [1.1, it shows that if almost all of the non-archimedean Euler factors of the zeta functions of two irreducible lattices $\Gamma_{1}$ and $\Gamma_{2}$ coincide, then $\alpha\left(\Gamma_{1}\right)=\alpha\left(\Gamma_{2}\right)$. This implies, for instance, to inner forms of groups of type $A_{n}$ defined over a number field. In the current paper we apply this to groups of type $A_{2}$.

In our third main theorem we determine the abscissae of convergence of the representation zeta functions of arithmetic groups of type $A_{2}$; cf. Appendix A for a precise definition of this class of groups. In the presence of the CSP, the representation zeta function of such an arithmetic group is an Euler product, whose non-archimedean factors are representation zeta functions of FAb compact $p$-adic analytic groups; see (1.1). Using our p-adic machinery and approximative Clifford theory, we prove the following global result.

Theorem C. Let $\Gamma$ be an arithmetic subgroup of a connected, simply connected simple algebraic group of type $A_{2}$ defined over a number field. If $\Gamma$ has the CSP, then $\alpha(\Gamma)=1$.

Remark 1.2. Naturally, Theorem C calls for a closer investigation of the singularity at $s=1$. In [3], we use a different approach to show that the zeta function of the special linear group $\mathrm{SL}_{3}(\mathcal{O})$ over the ring of integers $\mathcal{O}$ of a number field admits meromorphic continuation beyond $\operatorname{Re}(s)=1$ and that in this situation $s=1$ is a double pole. In fact, the assertion holds more generally for arithmetic groups of type ${ }^{1} A_{2}$ with the Congruence Subgroup Property.

A well-known conjecture of Serre asserts that an arithmetic subgroup of a connected, simply connected simple algebraic group has the CSP if and only if the latter is of 'higher rank'; cf. [46] and [43, Section 9.5]. Assuming this conjecture for groups of type $A_{2}$, Theorem $\mathrm{C}$ implies that Larsen and Lubotzky's conjecture, as stated at the end of Section 1.1, holds for higher-rank semisimple groups which are products of groups of type $A_{2}$. More precisely, one has 
Corollary D. Assuming Serre's conjecture, Larsen and Lubotzky's conjecture holds for groups $H=\prod_{i=1}^{r} \mathbf{G}_{i}\left(K_{i}\right)$, where each $K_{i}$ is a local field of characteristic 0 and each $\mathbf{G}_{i}$ is an absolutely almost simple $K_{i}$-group of type $A_{2}$ such that $\sum_{i=1}^{r} \operatorname{rk}_{K_{i}}\left(\mathbf{G}_{i}\right) \geq 2$ and none of the $\mathbf{G}_{i}\left(K_{i}\right)$ is compact.

Indeed, by Margulis' arithmeticity theorem any irreducible lattice $\Gamma$ in a semisimple group $H$ as in Corollary $\mathrm{D}$ is arithmetic, and Theorem $\mathrm{C}$ applies to such $\Gamma$; see [39. Chapter 7.2] and [35, Section 2] for details.

Key to our proof of Theorem $\mathbb{C}$ is the following local result, which we formulate in accordance with the notation introduced just before Theorem A. Recall in particular the definition of permissible $m$; cf. Definition 2.2.

Theorem E. Let $\mathfrak{o}$ be a compact discrete valuation ring of characteristic 0 whose residue field has cardinality $q$ and characteristic not equal to 3 . Let $\mathfrak{g}(\mathfrak{o})$ be one of the following two o-Lie lattices of type $A_{2}$ :

(a) $\mathfrak{s l}_{3}(\mathfrak{o})=\left\{\mathbf{x} \in \mathfrak{g l}_{3}(\mathfrak{o}) \mid \operatorname{Tr}(\mathbf{x})=0\right\}$,

(b) $\mathfrak{s u}_{3}(\mathfrak{O}, \mathfrak{o})=\left\{\mathbf{x} \in \mathfrak{s l}_{3}(\mathfrak{O}) \mid \mathbf{x}^{\sigma}=-\mathbf{x}^{\mathrm{t}}\right\}$, where $\mathfrak{O} \mid \mathfrak{o}$ is an unramified quadratic extension with non-trivial automorphism $\sigma$ and $\mathbf{x}^{\mathrm{t}}$ denotes the transpose of $\mathbf{x}$.

For $m \in \mathbb{N}$, let $\mathrm{G}^{m}(\mathfrak{o})$ be the $m$-th principal congruence subgroup of the corresponding group $\mathrm{SL}_{3}(\mathfrak{o})$ or $\mathrm{SU}_{3}(\mathfrak{O}, \mathfrak{o})$. Then, for all $m$ which are permissible for $\mathfrak{g}(\mathfrak{o})$, one has

$$
\zeta_{\mathrm{G}^{m}(\mathfrak{o})}(s)=q^{8 m} \frac{1+u(q) q^{-3-2 s}+u\left(q^{-1}\right) q^{-2-3 s}+q^{-5-5 s}}{\left(1-q^{1-2 s}\right)\left(1-q^{2-3 s}\right)},
$$

where

$$
u(X)=\left\{\begin{aligned}
X^{3}+X^{2}-X-1-X^{-1} & \text { if } \mathfrak{g}(\mathfrak{o})=\mathfrak{s l}_{3}(\mathfrak{o}), \\
-X^{3}+X^{2}-X+1-X^{-1} & \text { if } \mathfrak{g}(\mathfrak{o})=\mathfrak{s u} \mathfrak{u}_{3}(\mathfrak{O}, \mathfrak{o}) .
\end{aligned}\right.
$$

Remark 1.3. The Euler product (1.1) links the local analysis in TheoremE to the global results in Theorem $\mathrm{C}$ and its Corollary $\mathrm{D}$ for arithmetic groups of type $A_{2}$. In Theorem $\mathrm{E}$, the close resemblance between the representation zeta functions of the special linear and the special unitary groups is noteworthy and reminiscent of the Ennola duality for the characters of the finite groups $\mathrm{GL}_{n}\left(\mathbb{F}_{q}\right)$ and $\mathrm{GU}_{n}\left(\mathbb{F}_{q^{2}}, \mathbb{F}_{q}\right)$; cf. [32]. In [4, we compute explicitly the representation zeta functions of principal congruence subgroups of $\mathrm{SL}_{3}(\mathfrak{o})$, where $\mathfrak{o}$ is an unramified extension of $\mathbb{Z}_{3}$. The resulting formula is uniform in the residue field size, but genuinely different from the formulae in Theorem $\mathrm{E}$.

1.3. Discussion of results and techniques. The core of our technique is a p-adic formalism for the representation zeta functions of potent, saturable pro- $p$ groups. This approach has two key ingredients. Firstly, the Kirillov orbit method for potent, saturable pro- $p$ groups provides a way to construct the characters of irreducible complex representations in terms of co-adjoint orbits; see, e.g., [18, 19]. This 'linearisation' pioneered in [26, 31] - allows us to transform the original problem of enumerating representations by their dimension into the task of counting co-adjoint orbits by their size. We remark that the Kirillov orbit method can also be applied in the context of finitely generated nilpotent groups; see [25]. Voll used this method to study zeta functions which encode iso-twist classes of irreducible characters of finitely generated nilpotent groups; see [51]. These zeta functions were first studied by Hrushovski and Martin, who used sophisticated techniques from model theory and did not rely on the Kirillov orbit method; see [27.

The second main idea of our approach is to enumerate co-adjoint orbits by means of suitable $\mathfrak{p}$-adic integrals which are closely related to Igusa local zeta functions; cf. [12, 
29, 50]. A general class of such $\mathfrak{p}$-adic zeta functions was introduced in [51]; it does not rely on notions from model theory such as the concept of definable integrals utilised in [31, for which explicit formulae are not available. The key ingredient of our p-adic formalism is a description of the representation zeta functions of groups like $G^{m}(\mathfrak{O})$, which arise from a global o-Lie lattice $\Lambda$ as in the setup of Theorems $\mathrm{A}$ and $\mathrm{B}$, in terms of $\mathfrak{p}$-adic integrals of the shape

$$
z_{\mathfrak{O}}(r, t)=\int_{(x, \mathbf{y}) \in V(\mathfrak{D})}|x|_{\mathfrak{P}}^{t} \prod_{j=1}^{\lfloor d / 2\rfloor} \frac{\left\|F_{j}(\mathbf{y}) \cup F_{j-1}(\mathbf{y}) x^{2}\right\|_{\mathfrak{P}}^{r}}{\left\|F_{j-1}(\mathbf{y})\right\|_{\mathfrak{P}}^{r}} d \mu(x, \mathbf{y}),
$$

where $V(\mathfrak{O}) \subset \mathfrak{O}^{d+1}$ is a union of cosets modulo $\mathfrak{P}, \mu$ is the additive Haar measure on $\mathfrak{O}^{d+1}$, normalised so that $\mu\left(\mathfrak{O}^{d+1}\right)=1$, the $F_{j}(\mathbf{Y})$ are finite sets of polynomials over $\mathcal{O}$, which may be defined in terms of the structure constants of the $\mathcal{O}$-Lie lattice $\Lambda$ with respect to a given $\mathcal{O}$-basis, and we write $\|\cdot\|_{\mathfrak{P}}$ for the $\mathfrak{P}$-adic maximum norm; cf. (3.5)).

The proof of Theorem A relies heavily on deep algebro-geometric techniques and results which are commonly used to analyse integrals like (1.4). These include principalisations of ideals for the algebraic varieties defined by the polynomials in $F_{j}(\mathbf{Y})$, and aspects of the Weil conjectures regarding the zeta functions associated to smooth projective algebraic varieties over finite fields; see [12, 50]. Theorem A captures a threefold 'uniformity' of the representation zeta functions of the groups in question: regarding the variation of the prime, ring extension and congruence level. It is noteworthy that, in the setting of subring and subgroup growth, the impact of variation of all three parameters is much less understood than in the present context.

Of particular interest are Theorems $\mathrm{A}$ and $\mathrm{B}$ when they are applied to zeta functions associated to principal congruence subgroups of 'semisimple' compact $p$-adic analytic groups $\mathbf{G}\left(\mathcal{O}_{v}\right)$, featuring on the right hand side of the Euler product (1.1). In this context $\mathbf{G}$ is a connected, simply connected semisimple algebraic group defined over a number field $k$, with ring of integers $\mathcal{O}$. The Euler product links the local analysis in Theorems $\mathrm{A}, \mathrm{B}$ and $\mathrm{E}$ to the global result in Theorem $\mathrm{C}$ and its Corollary D Moreover, in the 'semisimple' case, we offer a Lie-theoretic interpretation of the varieties defined by the sets of polynomials $F_{j}(\mathbf{Y})$ occurring in (1.4), by identifying them with certain algebraic subvarieties of the Lie algebra associated to the group $\mathbf{G}$. These varieties are defined in terms of centraliser dimension, and yield a filtration of the locus of irregular elements in the Lie algebra. This makes them amenable to tools from algebraic Lie theory, for instance the theory of sheets; cf. [6].

Whilst Theorem $\mathrm{A}$ is a local result in as much as it concerns the representation zeta functions of pro- $p$ groups of the form $G^{m}(\mathfrak{O})$, we make use of the result's global framework to exclude finitely many places which require a refined analysis. It is an interesting question whether the phenomenon of (local) functional equations transcends the realm of pro- $p$ groups.

Question 1.4. Does a functional equation, akin to (1.2), hold for the (generic) nonarchimedean factors $\zeta_{\mathbf{G}\left(\mathcal{O}_{v}\right)}(s)$ occurring in the Euler product (1.1), or for the representation zeta functions of arithmetic groups?

Even in the cases where we have explicit formulae available (cf. [31, Theorem 7.5] and also [4] for $\mathrm{SL}_{2}(\mathfrak{o})$; cf. 3] for $\mathrm{SL}_{3}(\mathfrak{o})$ ), it is not clear which operation should play the role of the 'inversion of the prime' in the functional equations (1.2) of Theorem $\mathrm{A}$.

The proof of Theorem $\mathrm{B}$ relies on a refinement of the formulae which we utilise to deduce the functional equations (1.2) for 'generic' places. Indeed, the finite set $P$ of (real 
parts of) 'candidate poles' is obtained from the numerical data of a principalisation of ideals associated to the o-lattice $\Lambda$. In the case of good reduction modulo $\mathfrak{p}$, the inclusion in (11) follows from an explicit formula for the $\mathfrak{p}$-adic integrals describing $\zeta_{\mathfrak{G}^{m}(\mathfrak{O})}(s)$, which involves these numerical data. In the proof of Theorem $B$ we establish that $P$ is also a superset of the real parts of the poles in the case of bad reduction. Whilst it is in general a difficult problem to decide which of the 'candidate real parts' in $P$ are actually real parts of poles of a specific representation zeta function, the equality in (2) reflects that an irreducible quasi-projective variety defined over $\mathcal{O}$ has points modulo $\mathfrak{p}_{v}$ for a set of places $v$ of positive Dirichlet density. With a view toward the poles of the Euler factors in (1.1) we pose the following question.

Question 1.5. Let $G$ be a FAb compact $p$-adic analytic group, and $H \leq G$ an open subgroup. Is it the case that

$$
\left\{\operatorname{Re}(z) \mid z \text { a pole of } \zeta_{H}(s)\right\}=\left\{\operatorname{Re}(z) \mid z \text { a pole of } \zeta_{G}(s)\right\} ?
$$

We note that the maxima of these two finite sets coincide, because the abscissa of convergence of the representation zeta function of a rigid group is a commensurability invariant; see [35, Corollary 4.5].

In the proof of Theorem $\mathrm{C}$ we use standard results from the theory of orders in central simple algebras over number fields to show that almost all of the non-archimedean Euler factors of the zeta function $\zeta_{\Gamma}(s)$ of the arithmetic group $\Gamma$ are of the form $\zeta_{\mathrm{SL}_{3}(\mathfrak{o})}(s)$ or $\zeta_{\mathrm{SU}_{3}(\mathfrak{O}, \mathfrak{o})}(s)$. By Theorem E the abscissae of convergence of these local zeta functions are $2 / 3$. Theorem $B$ allows us to deduce that $2 / 3$ is also an upper bound for the local abscissae of convergence for the remaining finitely many exceptional places. In a second step we use approximative Clifford theory to 'lift' our analysis of representations of principal congruence subgroups to the encompassing groups $\mathrm{SL}_{3}(\mathfrak{o})$ and $\mathrm{SU}_{3}(\mathfrak{O}, \mathfrak{o})$, respectively. Whilst we do not produce explicit formulae for the representation zeta functions of these groups, our method is designed to retain enough control over suitable approximations to pin down the global abscissa of convergence. The formulae for the zeta functions of the groups $\mathrm{SL}_{3}(\mathfrak{o})$ given in our paper [3] are derived using an explicit analysis of similarity classes of $3 \times 3$-matrices over $\mathfrak{o}$.

It is noteworthy that, in the case of arithmetic groups $\Gamma$ of type $A_{2}$, the abscissa of convergence of the zeta function $\zeta_{\Gamma}(s)$ coincides with the abscissa of convergence of the Euler product of the zeta functions of the finite groups of Lie type $\mathbf{G}\left(\mathcal{O}_{v} / \mathfrak{p}_{v}\right)$; see, specifically, Proposition 7.9, Explicit computations of Euler products of finite groups of Lie type suggest, however, that the latter is a function of the Lie rank which tends to zero as the Lie rank tends to infinity. This does not yield the abscissa of convergence of $\zeta_{\Gamma}(s)$ as, by [35, Theorem 8.1], the abscissae of convergence of almost all of the local factors $\zeta_{\mathbf{G}\left(\mathcal{O}_{v}\right)}(s)$ are bounded away from zero.

The proof of Theorem $\mathrm{E}$ follows from a concrete application of our $\mathfrak{p}$-adic formalism and a description of the irregular locus in the complex Lie algebra $\mathfrak{s l}_{3}(\mathbb{C})$. The readiness with which our method can be applied in this concrete situation is remarkable; but, of course, it will be much more challenging to understand the algebraic varieties arising from higher dimensional groups. We remark that Theorem $\mathrm{E}$ also provides concrete examples of the general assertions made in Theorem $\mathrm{A}$ and illustrates some of the statements of Theorem B

A precursor and source of inspiration for the study of representation zeta functions is the area of subgroup growth; see [39]. Methods and tools from one subject area can be transferred to the other, and there is significant common ground, in particular regarding 
the class of 'semisimple' compact $p$-adic analytic groups. Theorems $\mathrm{A}$, Band $\mathrm{E}$ should be contrasted with our extremely limited knowledge regarding the subgroup zeta functions of 'semisimple' compact $p$-adic analytic pro- $p$ groups. For example, the subgroup zeta function of $\mathrm{SL}_{3}^{1}\left(\mathbb{Z}_{p}\right)$, or even just its pole spectrum, remains widely unknown; cf. [39, p. 431].

\subsection{Organisation and notation.}

1.4.1. Organisation of the paper. In the first part of the paper, we develop methods from $\mathfrak{p}$-adic integration to study representation zeta functions of certain compact $p$-adic analytic groups. One of the key tools facilitating this is the Kirillov orbit method. In Section 2, we review this method for potent and saturable pro- $p$ groups, together with some of the necessary background from $p$-adic Lie theory. In Section 3 we exhibit how the Kirillov orbit method allows us to transform the problem of computing representation zeta functions of the relevant groups into the problem of computing certain Poincaré series. These in turn are amenable to tools from the theory of $\mathfrak{p}$-adic integration developed in the study of Igusa local zeta functions and generalisations thereof. We utilise this approach to prove, in Section 4 . Theorems $\mathrm{A}$ and B. In Section 5 we explain a link between representation zeta functions of 'semisimple' $p$-adic analytic groups and a filtration of their associated Lie algebras, refining the loci of irregular elements.

In the second part of the paper we apply the $\mathfrak{p}$-adic formalism developed in the first part to the study of representation zeta functions of arithmetic groups of type $A_{2}$, and related pro- $p$ groups. We start, in Section 6, with the computation of the zeta functions of principal congruence subgroups of the compact $p$-adic analytic groups $\mathrm{SL}_{3}(\mathfrak{o})$ and $\mathrm{SU}_{3}(\mathfrak{O}, \mathfrak{o})$, thereby proving Theorem $\mathrm{E}$. The proof of Theorem $\mathrm{C}$ is given in Section 7 , The theorem is proved for groups of type ${ }^{1} A_{2}$ (inner forms) in Section 7.3 and for groups of type ${ }^{2} A_{2}$ (outer forms) in Section 7.4. We provide some background on Dirichlet generating functions in Section 7.1 and from Clifford theory in Section 7.2.

1.4.2. Notation. Throughout the paper, $p$ is a rational prime. We denote by $k$ a number field, with ring of integers 0 . For any non-archimedean place $v$ of $k$, we denote by $k_{v}$ the completion of $k$ with respect to $v$ and by $\mathcal{O}_{v}$ the corresponding complete valuation ring, with maximal ideal $\mathfrak{p}_{v}$ and residue field $\mathbb{F}_{q_{v}}=\mathcal{O}_{v} / \mathfrak{p}_{v}$ of size $q_{v}$. For any finite set $S$ of places of $k$, including all the archimedean ones, we write $\mathcal{O}_{S}:=\left\{x \in k \mid \forall v \notin S: x \in \mathcal{O}_{v}\right\}$ for the ring of $S$-integers in $k$. We denote by $\Lambda$ an o-Lie lattice. A finite extension field of $k$ is typically denoted by $K$, its ring of integers $\mathcal{O}$, etc.

The field of $p$-adic numbers is denoted by $\mathbb{Q}_{p}$, the ring of $p$-adic integers by $\mathbb{Z}_{p}$. More generally, $\mathfrak{k}$ denotes a $\mathfrak{p}$-adic field, i.e. a non-archimedean local field of characteristic 0 . By $\mathfrak{o}$ we denote a compact discrete valuation ring of characteristic 0 and residue field characteristic $p$; typically $\mathfrak{o}$ is the ring of integers of the $\mathfrak{p}$-adic field $\mathfrak{k}$. The maximal ideal of $\mathfrak{o}$ is denoted by $\mathfrak{p}=\pi \mathfrak{o}$, where $\pi$ is a chosen uniformiser. We write $\mathbb{F}_{q}$ for the residue field $\mathfrak{o} / \mathfrak{p}$ of cardinality $q=p^{f}$, where $f=f\left(\mathfrak{k}, \mathbb{Q}_{p}\right)=f\left(\mathfrak{o}, \mathbb{Z}_{p}\right)$ is the (absolute) degree of inertia.

For $x \in \mathfrak{k} \backslash\{0\}$, we write $|x|_{\mathfrak{p}}$ to denote the $\mathfrak{p}$-adic absolute value of $x$, normalised so that $|\pi|_{\mathfrak{p}}=q^{-1}$. For a finite set $X \subseteq \mathfrak{k}$ we set $\|X\|_{\mathfrak{p}}:=\max \left\{|x|_{\mathfrak{p}} \mid x \in X\right\}$. We write $\mu$ for the additive Haar measure on spaces like $\mathfrak{o}^{n}$, normalised so that $\mu\left(\mathfrak{o}^{n}\right)=1$. A finite extension field of $\mathfrak{k}$ is denoted by $\mathfrak{K}$, its ring of integers by $\mathfrak{O}$ with maximal ideal $\mathfrak{P}$. We write $f(\mathfrak{K}, \mathfrak{k})=f(\mathfrak{O}, \mathfrak{o})$ for the (relative) degree of inertia and $e(\mathfrak{K}, \mathfrak{k})=e(\mathfrak{O}, \mathfrak{o})$ for the (relative) ramification index. 
We give a short pictorial summary of the global and local notation.

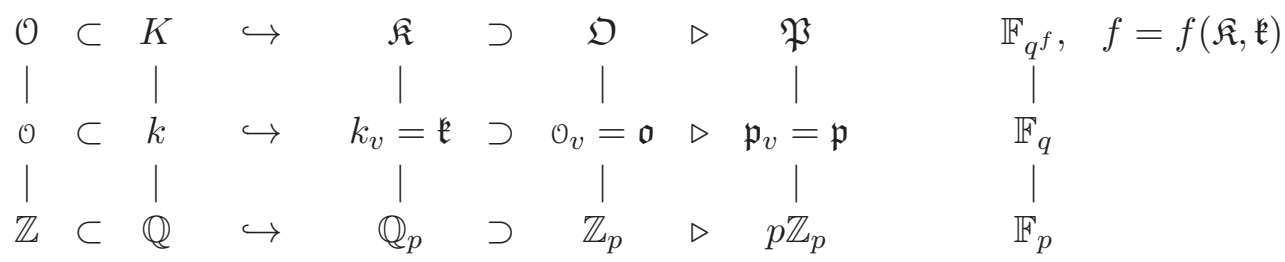

Typically, we use the usual notation, like $\mathbb{N}^{n}, \mathfrak{o}^{n}, \mathbb{F}_{q}^{n}$ etc., to denote Cartesian powers. However, in cases where this notation could be misunderstood we make a small modification. For instance, we write $\mathfrak{p}^{n}$ to denote the $n$-th power of the maximal ideal $\mathfrak{p}$, and $\mathfrak{p}^{(n)}$ for the $n$-fold Cartesian power $\times_{i=1}^{n} \mathfrak{p}$. The transpose of a matrix $M$ is denoted by $M^{\mathrm{t}}$. The non-trivial Galois automorphism of a quadratic field extension $\mathfrak{K} \mid \mathfrak{k}$ is denoted by $\sigma$, the resulting standard involution on the matrix algebra $\operatorname{Mat}_{n}(\mathfrak{K})$ by $\circ$, i.e. $\mathbf{x}^{\circ}:=\left(\mathbf{x}^{\sigma}\right)^{\mathrm{t}}$.

We write $F^{*}$ to denote the multiplicative group of a field $F$ and extend this notation as follows. For any non-trivial o-module $M$ we write $M^{*}:=M \backslash \mathfrak{p} M$. For the trivial $\mathfrak{o}$-module $\{0\}$ we define $\{0\}^{*}:=\{0\}$. The Pontryagin dual of a compact abelian group $\mathfrak{a}$, e.g. the additive group of an $\mathfrak{o}$-module, is $\widehat{\mathfrak{a}}:=\operatorname{Irr}(\mathfrak{a}):=\operatorname{Hom}_{\mathbb{Z}}^{\text {cont }}\left(\mathfrak{a}, \mathbb{C}^{*}\right)$. More generally, we write $\widehat{G}=\operatorname{Irr}(G)$ for the collection of continuous, irreducible complex characters of a profinite group $G$. If $N \unlhd G$, the inertia group of $\vartheta \in \operatorname{Irr}(N)$ in $G$ is denoted by $I_{G}(\vartheta)$. The following list collects further key notation, together with the number of the section where it is introduced.

\begin{tabular}{r||l|l}
$\alpha(\Gamma)$ & abscissa of convergence & 1.1 \\
$\operatorname{Irr}_{n}(\mathfrak{h})$ & characters of level $n$ & 2.2 \\
$\mathcal{R}_{\mathfrak{g}, \mathbf{b}}(\mathbf{Y})$ & commutator matrix & 3.1 \\
$W(\mathfrak{o})=\left(\mathfrak{o}^{d}\right)^{*}$ & region of integration & 3.1 \\
$\mathcal{P}_{\mathcal{R}, \mathfrak{o}}(s)$ & Poincaré series & 3.1 \\
$\mathcal{Z}_{\mathfrak{o}}(r, t)$ & $\mathfrak{p}$-adic integral & 3.2 \\
$\rho$ & maximal rank & 3.2 \\
$\mathcal{V}_{i}, \mathcal{W}_{i}$ & varieties in stratifications & 5 \\
$\kappa_{0}$ & normalised Killing form & 5
\end{tabular}

\section{Part 1. p-Adic formalism}

\section{2. $p$-AdiC ANALYTIC PRO- $p$ GROUPS AND The KiRILlOV ORBIT METHOD}

Much of the theory of compact $p$-adic analytic pro- $p$ groups can be developed satisfactorily by using the concept of uniformly powerful pro- $p$ groups; see [14. However, for a more complete picture of $p$-adic Lie theory it is advantageous to work with saturable pro- $p$ groups; see [18, 34].

2.1. The notion of saturability goes back to Lazard and is based on valuation maps; see [36, 34]. In [18, González-Sánchez has given the following useful characterisation. A finitely generated pro- $p$ group $G$ is saturable if and only if it is torsion-free and admits a potent filtration, i.e. a descending series $G_{i}, i \in \mathbb{N}$, of normal subgroups of $G$ such that (i) $G=G_{1}$, (ii) $\bigcap_{i \in \mathbb{N}} G_{i}=1$, (iii) $\left[G_{i}, G\right] \subseteq G_{i+1}$ and $\left[G_{i, p-1} G\right] \subseteq G_{i+1}^{p}$ for all $i \in \mathbb{N}$. (Here $\left[G_{i, p-1} G\right]$ denotes the left-normed iterated commutator with one occurrence of $G_{i}$ and $p-1$ occurrences of $G$, and $G_{i+1}^{p}$ stands for the group generated by $p$-th powers of elements of $G_{i+1}$.) Uniformly powerful pro- $p$ groups and, more generally, torsion-free finitely generated pro- $p$ groups $G$ with $\gamma_{p}(G) \subseteq \Phi(G)^{p}$ are saturable. In [20] it is shown 
that every torsion-free $p$-adic analytic pro- $p$ group of dimension less than $p$ is saturable. Klopsch proved that every insoluble maximal $p$-adic analytic just-infinite pro- $p$ group of dimension less than $p-1$ is saturable; see 34 . These groups occur naturally as maximal open pro- $p$ subgroups of automorphism groups of semisimple $p$-adic Lie algebras and they provide a rich class of groups whose representation zeta functions are of considerable interest.

To a saturable pro- $p$ group $G$ one associates a saturable $\mathbb{Z}_{p}$-Lie lattice $\mathfrak{g}=\log (G)$, which coincides with $G$ as a topological space, and there is a tight Lie correspondence between $G$ and $\mathfrak{g}$; see [20]. The simplest way to recover the group $G$ from its $\mathbb{Z}_{p}$-Lie lattice $\mathfrak{g}$ is by defining a group multiplication on $\mathfrak{g}$ via the Hausdorff series. When we start from a saturable $\mathbb{Z}_{p}$-Lie lattice $\mathfrak{g}$, we denote this group by $\exp (\mathfrak{g})$. The following straightforward proposition characterises saturable pro- $p$ groups which are FAb, i.e. which have the property that every open subgroup has finite abelianisation.

Proposition 2.1. Let $G$ be a saturable pro-p group and $\mathfrak{g}=\log (G)$ the associated saturable $\mathbb{Z}_{p}$-Lie lattice. Then the following are equivalent.

(1) $G$ is FAb.

(2) $G$ has finite abelianisation $G /[G, G]$.

(3) $\mathfrak{g}$ has finite abelianisation $\mathfrak{g} /[\mathfrak{g}, \mathfrak{g}]$.

(4) $\mathbb{Q}_{p} \otimes \mathfrak{g}$ is a perfect $\mathbb{Q}_{p}$-Lie algebra.

Proof. Everything follows from the observation that the terms of the derived series of the group $G$ and of the Lie lattice $\mathfrak{g}$ coincide as sets; see [18, Corollary 4.7].

We remark that a compact $p$-adic analytic group is FAb if and only if it has an open FAb saturable pro- $p$ subgroup.

In applying the Kirillov orbit method, we will be dealing with potent $\mathbb{Z}_{p}$-Lie lattices and pro- $p$ groups. We recall that a $\mathbb{Z}_{p}$-Lie lattice $\mathfrak{g}$ is potent if $\gamma_{p-1}(\mathfrak{g}) \subseteq p \mathfrak{g}$ for $p>2$ and $\gamma_{2}(\mathfrak{g}) \subseteq 4 \mathfrak{g}$ for $p=2$; similarly, a pro- $p$ group $G$ is potent if $\gamma_{p-1}(G) \subseteq G^{p}$ for $p>2$ and $\gamma_{2}(G) \subseteq G^{4}$ for $p=2$. If $G$ is a saturable pro- $p$ group and $\mathfrak{g}$ the associated $\mathbb{Z}_{p}$-Lie lattice, then $G$ is potent if and only if $\mathfrak{g}$ is potent. Saturable pro- $p$ groups of dimension less than $p$ are potent. In particular, the main theorem in [20] shows that every torsion-free $p$-adic analytic pro- $p$ group of dimension less than $p$ is saturable and potent.

Definition 2.2. Let $\mathfrak{o}$ be a compact discrete valuation ring of characteristic 0 and residue field characteristic $p$, and let $\mathfrak{g}$ be an $\mathfrak{o}$-Lie lattice. For $m \in \mathbb{N}_{0}$, let $\mathfrak{g}^{m}:=\mathfrak{p}^{m} \mathfrak{g}$, where $\mathfrak{p}$ denotes the maximal ideal of $\mathfrak{o}$. We call $m \in \mathbb{N}_{0}$ permissible for $\mathfrak{g}$ if $\mathfrak{g}^{m}$ is potent and saturable as a $\mathbb{Z}_{p}$-Lie lattice.

The following effective result shows that, for a given $\mathfrak{o}$-lattice $\mathfrak{g}$, almost all nonnegative integers are permissible.

Proposition 2.3. Let $\mathfrak{o}$ be a compact discrete valuation ring of characteristic 0 and residue field characteristic $p$, and let $\mathfrak{g}$ be an $\mathfrak{o}$-Lie lattice. Let $m \in \mathbb{N}_{0}$ and let $e:=$ $e\left(\mathfrak{o}, \mathbb{Z}_{p}\right)$ be the absolute ramification index of $\mathfrak{o}$.

If $m>e(p-1)^{-1}$, then $\mathfrak{g}^{m}$ is saturable. Moreover, if $p>2$ and $m \geq e(p-2)^{-1}$, then $\mathfrak{g}^{m}$ is potent. If $p=2$ and $m \geq 2 e$, then $\mathfrak{g}^{m}$ is potent.

Proof. Suppose that $m>e(p-1)^{-1}$. For $x \in \mathfrak{g}^{m} \backslash\{0\}$ define $w(x):=\frac{1}{e} \max \{n \in \mathbb{N} \mid$ $\left.x \in \mathfrak{g}^{n}\right\}$, and put $w(0):=\infty$. Then the map $w: \mathfrak{g}^{m} \rightarrow \mathbb{R}_{>0} \cup\{\infty\}$ satisfies Lazard's condition for a valuation map, summarised in [34, Section 2], and $\mathfrak{g}^{m}$ is saturable. 
Suppose that $p>2$ and $m \geq e(p-2)^{-1}$. Then $\gamma_{p-1}\left(\mathfrak{g}^{m}\right) \subseteq \mathfrak{g}^{m(p-1)} \subseteq \mathfrak{g}^{e+m}=p \mathfrak{g}^{m}$, hence $\mathfrak{g}^{m}$ is potent.

Suppose that $p=2$ and $m \geq 2 e$. Then $\left[\mathfrak{g}^{m}, \mathfrak{g}^{m}\right] \subseteq \mathfrak{g}^{2 m} \subseteq \mathfrak{g}^{2 e+m}=4 \mathfrak{g}^{m}$, hence $\mathfrak{g}^{m}$ is potent.

Note in particular that, if $e\left(\mathfrak{o}, \mathbb{Z}_{p}\right)=1$, then for $p>2$ every $m \geq 1$ is permissible for every $\mathfrak{o}$-Lie lattice $\mathfrak{g}$, and, similarly, for $p=2$ every $m \geq 2$ is permissible.

2.2. The Kirillov orbit method in the context of compact $p$-adic analytic groups was first developed by Howe in [26]. Jaikin-Zapirain applied the theory to study representation zeta functions of FAb compact p-adic analytic groups and extended Howe's work; see [31. A useful description of the Kirillov orbit method for potent saturable pro- $p$ groups can be found in [19].

Let $G$ be a FAb potent saturable pro- $p$ group and $\mathfrak{g}$ the associated potent saturable $\mathbb{Z}_{p}$-Lie lattice. We denote by $\widehat{\mathfrak{g}}:=\operatorname{Irr}(\mathfrak{g})$ the Pontryagin dual of the compact abelian group $(\mathfrak{g},+)$, viz. the group $\operatorname{Hom}_{\mathbb{Z}}^{\text {cont }}\left(\mathfrak{g}, \mathbb{C}^{*}\right)=\operatorname{Hom}_{\mathbb{Z}}^{\text {cont }}\left(\mathfrak{g}, \mu_{p^{\infty}}\right)$ of continuous irreducible complex characters of the additive group $\mathfrak{g}$, where $\mu_{p} \cong \mathbb{Q}_{p} / \mathbb{Z}_{p}$ stands for the group of complex roots of unity of $p$-power order. To $\omega \in \widehat{\mathfrak{g}}$ we associate the bi-additive and antisymmetric form

$$
b_{\omega}: \mathfrak{g} \times \mathfrak{g} \rightarrow \mu_{p^{\infty}}, \quad b_{\omega}(x, y):=\omega([x, y]) .
$$

The radical of the form $b_{\omega}$ is

$$
\operatorname{Rad}(\omega):=\operatorname{Rad}\left(b_{\omega}\right)=\left\{x \in \mathfrak{g} \mid \forall y \in \mathfrak{g}: b_{\omega}(x, y)=1\right\} .
$$

According to [31, Corollary 2.13] and [19, Theorem 5.2] we have

$$
\zeta_{G}(s)=\sum_{\omega \in \operatorname{Irr}(\mathfrak{g})}|\mathfrak{g}: \operatorname{Rad}(\omega)|^{-(s+2) / 2} .
$$

Next suppose that $\mathfrak{g}$ is an $\mathfrak{o}$-Lie lattice, where $\mathfrak{o}$ is a compact discrete valuation ring of characteristic 0 and residue field characteristic $p$. Suppose that $m \in \mathbb{N}_{0}$ is permissible for $\mathfrak{g}$. In order to make effective use of the Kirillov orbit method in studying the representation zeta function of the $p$-adic analytic pro- $p$ group $\mathrm{G}^{m}=\exp \left(\mathfrak{g}^{m}\right)$, we recall a basic description of the dual $\widehat{\mathfrak{g}^{m}}$ of $\mathfrak{g}^{m}$, viewed as a $\mathbb{Z}_{p}$-module, in terms of $\mathfrak{o}$-functionals of $\mathfrak{g}^{m}$, viewed as an $\mathfrak{o}$-module.

Lemma 2.4. The dual $\widehat{\mathfrak{h}}$ of an $\mathfrak{o}$-Lie lattice $\mathfrak{h}$ can be written as a disjoint union

$$
\widehat{\mathfrak{h}}=\bigcup_{n \in \mathbb{N}_{0}} \operatorname{Irr}_{n}(\mathfrak{h}), \quad \text { where } \operatorname{Irr}_{n}(\mathfrak{h}) \cong \operatorname{Hom}_{\mathfrak{o}}\left(\mathfrak{h}, \mathfrak{o} / \mathfrak{p}^{n}\right)^{*}
$$

Proof. Let $\mathfrak{k}$ denote the field of fractions of $\mathfrak{o}$, which is a finite extension of $\mathbb{Q}_{p}$. The trace map induces a non-degenerate $\mathbb{Q}_{p}$-bilinear form $\mathfrak{k} \times \mathfrak{k} \rightarrow \mathbb{Q}_{p},(x, y) \mapsto \operatorname{Tr}_{\mathfrak{k} \mid \mathbb{Q}_{p}}(x y)$. The codifferent of $\mathfrak{k} \mid \mathbb{Q}_{p}$ is the fractional ideal consisting of all $x \in \mathfrak{k}$ such that $\operatorname{Tr}_{\mathfrak{k} \mid \mathbb{Q}_{p}}(x y) \in \mathbb{Z}_{p}$ for all $y \in \mathfrak{o}$. The different $\mathfrak{D}_{\mathfrak{k} \mid \mathbb{Q}_{p}}$ of the extension $\mathfrak{k} \mid \mathbb{Q}_{p}$ is the inverse of the codifferent and thus an ideal of $\mathfrak{o}$, say $\mathfrak{p}^{\delta}$. Regarding the parameter $\delta=\delta(\mathfrak{k}) \in \mathbb{N}_{0}$, it is known that $\delta \geq e\left(\mathfrak{k}, \mathbb{Z}_{p}\right)-1$ with equality if and only if $e\left(\mathfrak{k}, \mathbb{Q}_{p}\right)$ is prime to $p$; see [47, Chapter III]. The Pontryagin dual of $(\mathfrak{k},+)$ is $\widehat{\mathfrak{k}}:=\operatorname{Hom}_{\mathbb{Z}}^{\text {cont }}\left(\mathfrak{k}, \mathbb{C}^{*}\right)=\operatorname{Hom}_{\mathbb{Z}}^{\text {cont }}\left(\mathfrak{k}, \mu_{p} \infty\right)$. Via the Tate character

$$
\chi: \mathfrak{k} \stackrel{\operatorname{Tr}_{\mathfrak{k} \mid \mathbb{Q}_{p}}}{\longrightarrow} \mathbb{Q}_{p} \longrightarrow \mathbb{Q}_{p} / \mathbb{Z}_{p} \stackrel{\cong}{\longrightarrow} \mu_{p^{\infty}}
$$

one obtains an isomorphism

$$
\mathfrak{k} \rightarrow \widehat{\mathfrak{k}}, \quad x \mapsto \chi_{x}, \quad \text { where } \chi_{x}: \mathfrak{k} \rightarrow \mu_{p}, y \mapsto \chi(x y),
$$


showing that $\mathfrak{k}$ is self-dual; cf. [10, Chapter XV, Section 2.2]. Let $\mathfrak{p}=\pi \mathfrak{o}$. From $\mathfrak{D}_{\mathfrak{k} \mid \mathbb{Q}_{p}}=\mathfrak{p}^{\delta}=\pi^{\delta} \mathfrak{o}$ one obtains, for each $n \in \mathbb{N}_{0}$, a (non-canonical) pairing of finite abelian groups

$$
\left(\mathfrak{o} / \mathfrak{p}^{n}\right) \times\left(\mathfrak{p}^{-n} / \mathfrak{o}\right) \rightarrow \mu_{p}, \quad\left(x+\mathfrak{p}^{n}, y+\mathfrak{o}\right) \mapsto \chi\left(\pi^{-\delta} x y\right) .
$$

As $\mathfrak{h}$ is a free $\mathfrak{o}$-module of finite rank, this translates readily into an isomorphism of the finite abelian groups $\widehat{\mathfrak{h} / \mathfrak{p}^{n} \mathfrak{h}}$ and $\mathfrak{p}^{-n} \mathfrak{h} / \mathfrak{h}$. On the other hand there is, of course, for each $n \in \mathbb{N}_{0}$ the (non-canonical) isomorphism of $\mathfrak{o}$-modules $\mathfrak{p}^{-n} \mathfrak{h} / \mathfrak{h} \rightarrow \mathfrak{h} / \mathfrak{p}^{n} \mathfrak{h}$, induced by multiplication by $\pi^{n}$, and a natural pairing of $\mathfrak{o}$-modules

$$
\left(\mathfrak{h} / \mathfrak{p}^{n} \mathfrak{h}\right) \times \operatorname{Hom}_{\mathfrak{o}}\left(\mathfrak{h} / \mathfrak{p}^{n} \mathfrak{h}, \mathfrak{o} / \mathfrak{p}^{n}\right) \rightarrow \mathfrak{o} / \mathfrak{p}^{n}
$$

showing that $\mathfrak{h} / \mathfrak{p}^{n} \mathfrak{h} \cong \operatorname{Hom}_{\mathfrak{o}}\left(\mathfrak{h}, \mathfrak{o} / \mathfrak{p}^{n}\right)$. We thus obtain

$$
\widehat{\mathfrak{h}}=\bigcup_{n \in \mathbb{N}_{0}} \widehat{\mathfrak{h} / \mathfrak{p}^{n} \mathfrak{h}} \cong \bigcup_{n \in \mathbb{N}_{0}}\left(\mathfrak{p}^{-n} \mathfrak{h} / \mathfrak{h}\right)^{*} \cong \bigcup_{n \in \mathbb{N}_{0}}\left(\mathfrak{h} / \mathfrak{p}^{n} \mathfrak{h}\right)^{*} \cong \bigcup_{n \in \mathbb{N}_{0}} \operatorname{Hom}_{\mathfrak{o}}\left(\mathfrak{h}, \mathfrak{o} / \mathfrak{p}^{n}\right)^{*}
$$

From the proof of the lemma we observe that, in the case $\mathfrak{h}=\mathfrak{g}^{m}$, the description of $\widehat{\mathfrak{g}^{m}}$ in terms of $\mathfrak{o}$-functionals is $\mathrm{G}^{m}$-equivariant so that one can accurately describe the

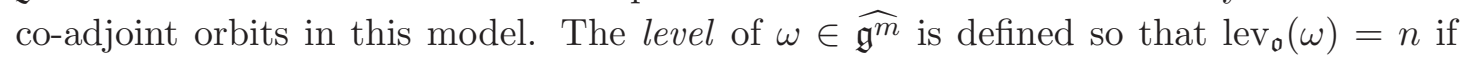
$\omega \in \operatorname{Irr}_{n}\left(\mathfrak{g}^{m}\right)$. If $\mathfrak{o}$ is unramified, the level of any $\omega \in \widehat{\mathfrak{g}^{m}}$ is simply the logarithm to base $p$ of its order, viz. $\min \left\{n \in \mathbb{N}_{0} \mid \omega\left(p^{n} \mathfrak{g}^{m}\right)=1\right\}$.

\section{Poincaré SERIES AND $\mathfrak{p}$-ADIC FORMALISM}

Let $\mathfrak{o}$ be a compact discrete valuation ring of characteristic 0 , with maximal ideal $\mathfrak{p}=\pi \mathfrak{o}$, field of fractions $\mathfrak{k}$ and residue field $\mathfrak{o} / \mathfrak{p}$ of cardinality $q$ and characteristic $p$. Let $\mathfrak{g}$ be an $\mathfrak{o}$-Lie lattice such that $\mathfrak{k} \otimes_{\mathfrak{o}} \mathfrak{g}$ is perfect, with $\operatorname{dim}_{\mathfrak{k}}\left(\mathfrak{k} \otimes_{\mathfrak{o}} \mathfrak{g}\right)=d$, say. According to Sections 2.1 and 2.2, for every sufficiently large $m \in \mathbb{N}_{0}$, the $m$-th principal congruence $\mathfrak{o}$-Lie sublattice $\mathfrak{g}^{m}:=\mathfrak{p}^{m} \mathfrak{g}$ of $\mathfrak{g}$ corresponds to a FAb $p$-adic analytic pro- $p$ group $\mathrm{G}^{m}=\exp \left(\mathfrak{g}^{m}\right)$, whose irreducible complex characters can be captured by means of the Kirillov orbit method. Our aim in this section is to express the zeta functions $\zeta_{\mathrm{G}^{m}}(s)$ in terms of a suitable Poincaré series and to derive an integral formula, connecting $\zeta_{\mathrm{G}^{m}}(s)$ to Igusa local zeta functions. Our treatment makes use of the underlying o-module structure of the Lie lattice $\mathfrak{g}$ and reveals that the dependence of $\zeta_{\mathrm{G}^{m}}(s)$ on $m$ can be captured in a simple factor $q^{d m}$.

3.1. Poincaré series. In this section we set up the Poincaré series which helps us to establish, for each permissible $m$, a link between the representation zeta function of the group $\mathrm{G}^{m}$ and a suitable generalised Igusa local zeta function; see Proposition 3.1 .

We fix an $\mathfrak{o}$-basis $\mathbf{b}:=\left(b_{1}, \ldots, b_{d}\right)$ for the $\mathfrak{o}$-Lie lattice $\mathfrak{g}$. The structure constants $\lambda_{i j}^{h}$ of the $\mathfrak{o}$-Lie lattice $\mathfrak{g}$ with respect to the basis $\mathbf{b}$ are encoded in the commutator matrix

$$
\mathcal{R}(\mathbf{Y}):=\mathcal{R}_{\mathfrak{g}, \mathbf{b}}(\mathbf{Y})=\left(\sum_{h=1}^{d} \lambda_{i j}^{h} Y_{h}\right)_{i j} \in \operatorname{Mat}_{d}(\mathfrak{o}[\mathbf{Y}])
$$

whose entries are linear forms in independent variables $Y_{1}, \ldots, Y_{d}$. Clearly, for any $m \in \mathbb{N}_{0}$, the commutator matrix of the $\mathfrak{o}$-Lie lattice $\mathfrak{g}^{m}$ with respect to the shifted basis $\pi^{m} \mathbf{b}$ is $\pi^{m} \mathcal{R}(\mathbf{Y})$.

We write $W(\mathfrak{o}):=\left(\mathfrak{o}^{d}\right)^{*}$ and consider the finite affine cones

$$
W_{n}(\mathfrak{o}):=\left(W(\mathfrak{o})+\left(\mathfrak{p}^{n}\right)^{(d)}\right) /\left(\mathfrak{p}^{n}\right)^{(d)}=\left(\left(\mathfrak{o} / \mathfrak{p}^{n}\right)^{d}\right)^{*}, \quad n \in \mathbb{N}_{0} .
$$


Let $\mathbf{y} \in W(\mathfrak{o})$. Note that $\mathcal{R}(\mathbf{y})$ is an antisymmetric $d \times d$ matrix over $\mathfrak{o}$. Therefore its elementary divisors are of the form $\mathfrak{p}^{a}$ with $a \in \mathbb{N}_{0} \cup\{\infty\}$. Moreover, if $d$ is even, they can be arranged in pairs. If $d$ is odd, they also come in pairs, except for a single extra divisor equal to $\mathfrak{p}^{\infty}=\{0\}$. Define

$$
\nu(\mathcal{R}(\mathbf{y})):=\left(a_{1}, \ldots, a_{\lfloor d / 2\rfloor}\right) \in\left(\mathbb{N}_{0} \cup\{\infty\}\right)^{\lfloor d / 2\rfloor},
$$

where $0 \leq a_{1} \leq \ldots \leq a_{|d / 2|}$ and $\mathcal{R}(\mathbf{y})$ is congruent to the block-diagonal matrix comprised of $2 \times 2$ blocks $\left(\begin{array}{cc}0 & \pi^{a_{i}} \\ -\pi^{a_{i}} & 0\end{array}\right), i \in\{1, \ldots,\lfloor d / 2\rfloor\}$, and a single $1 \times 1$ block (0) if $d$ is odd. (Two matrices $A$ and $B$ over a ring $R$ are said to be congruent if there exist invertible matrices $S$ and $T$ over $R$ such that $S A T=B$. If $A, B$ are antisymmetric, one may take $T=S^{\mathrm{t}}$.)

Now let $n \in \mathbb{N}_{0}$ and let $\overline{\mathbf{y}}$ denote the image of $\mathbf{y}$ in $W_{n}(\mathfrak{o})$. Then $\mathcal{R}(\overline{\mathbf{y}})$ is an antisymmetric $d \times d$ matrix over $\mathfrak{o} / \mathfrak{p}^{n}$ and the valuations of its elementary divisors are encoded by the tuple

$$
\nu(\mathcal{R}(\overline{\mathbf{y}})):=\nu_{n}(\mathcal{R}(\mathbf{y})):=\left(\min \left\{a_{i}, n\right\}\right)_{i \in\{1, \ldots,\lfloor d / 2\rfloor\}} \in\{0,1, \ldots, n\}^{\lfloor d / 2\rfloor} .
$$

The central counting function entering into the picture is

$$
\mathcal{N}_{n, \mathbf{a}}^{\mathfrak{o}}:=\#\left\{\overline{\mathbf{y}} \in W_{n}(\mathfrak{o}) \mid \nu(\mathcal{R}(\overline{\mathbf{y}}))=\mathbf{a}\right\}, \quad(n, \mathbf{a}) \in \mathbb{N}_{0} \times \mathbb{N}_{0}^{\lfloor d / 2\rfloor} .
$$

It gives rise to the associated Poincaré series

$$
\mathcal{P}_{\mathcal{R}, \mathfrak{o}}(s):=\sum_{n \in \mathbb{N}_{0}, \mathbf{a} \in \mathbb{N}_{0}^{\lfloor d / 2\rfloor}} \mathcal{N}_{n, \mathbf{a}}^{\mathfrak{o}} q^{-\left(\sum_{i=1}^{\lfloor d / 2\rfloor}\left(n-a_{i}\right)\right) s} .
$$

We note that $\mathcal{N}_{n, \mathbf{a}}^{\mathfrak{o}} \neq 0$ implies $0 \leq a_{1} \leq \ldots \leq a_{\lfloor d / 2\rfloor} \leq n$.

The principal aim of this section is to establish a link between the representation zeta functions of the groups $\mathrm{G}^{m}$ associated to the Lie lattices $\mathfrak{g}^{m}$ for permissible $m$ and the Poincaré series defined in (3.3).

Proposition 3.1. Let $\mathfrak{g}$ be as defined at the beginning of Section [3. Then for all $m \in \mathbb{N}_{0}$ which are permissible for $\mathfrak{g}$ we have

$$
\zeta_{\mathrm{G} m}(s)=q^{d m} \mathcal{P}_{\mathcal{R}, \mathfrak{o}}(s+2) .
$$

As an immediate consequence, we observe

Corollary 3.2. For every permissible $m \in \mathbb{N}_{0}$, the representation zeta function $\zeta_{\mathrm{G}^{m}}(s)$ is a power series in $q^{-s}$, i.e. the character degrees of $\mathrm{G}^{m}$ are powers of $q$.

The proof of Proposition 3.1 requires some preparation. In order to apply the Kirillov orbit method we consider the space $\widehat{\mathfrak{g}^{m}}$ which, according to Section 2.2, decomposes as $\widehat{\mathfrak{g}^{m}}=\dot{\bigcup}_{n \in \mathbb{N}_{0}} \operatorname{Irr}_{n}\left(\mathfrak{g}^{m}\right)$ where $\operatorname{Irr}_{n}\left(\mathfrak{g}^{m}\right)=\left\{\omega \in \widehat{\mathfrak{g}^{m}} \mid \operatorname{lev}_{\mathfrak{o}}(\omega)=n\right\} \cong \operatorname{Hom}_{\mathfrak{o}}\left(\mathfrak{g}^{m}, \mathfrak{o} / \mathfrak{p}^{n}\right)^{*}$. Moreover, this description is $\mathrm{G}^{m}$-equivariant. Note that for every $n \in \mathbb{N}$ there is a natural surjection

$$
\operatorname{Hom}_{\mathfrak{o}}\left(\mathfrak{g}^{m}, \mathfrak{o}\right)^{*} \rightarrow \operatorname{Hom}_{\mathfrak{o}}\left(\mathfrak{g}^{m}, \mathfrak{o} / \mathfrak{p}^{n}\right)^{*} \cong \operatorname{Irr}_{n}\left(\mathfrak{g}^{m}\right) .
$$

We say that $w \in \operatorname{Hom}_{\mathfrak{o}}\left(\mathfrak{g}^{m}, \mathfrak{o}\right)^{*}$ is a representative of $\omega \in \widehat{\mathfrak{g}^{m}}$ if the image of $w$ under this surjection corresponds to $\omega$.

The chosen $\mathfrak{o}$-basis $\mathbf{b}$ for $\mathfrak{g}$ yields a shifted basis $\pi^{m} \mathbf{b}$ for $\mathfrak{g}^{m}$ and thus an explicit co-ordinate system

$$
\mathfrak{g}^{m} \stackrel{\simeq}{\rightarrow} \mathfrak{o}^{d}, \quad z=\sum_{i=1}^{d} z_{i}\left(\pi^{m} b_{i}\right) \mapsto \underline{z}=\left(z_{1}, \ldots, z_{d}\right) .
$$


Likewise, the dual $\mathfrak{o}$-basis $\mathbf{b}^{\vee}$ for $\operatorname{Hom}_{\mathfrak{o}}(\mathfrak{g}, \mathfrak{o})$ and its shift $\pi^{-m} \mathbf{b}^{\vee}$ for $\operatorname{Hom}_{\mathfrak{o}}\left(\mathfrak{g}^{m}, \mathfrak{o}\right)$ give a co-ordinate system

$$
\operatorname{Hom}_{\mathfrak{o}}\left(\mathfrak{g}^{m}, \mathfrak{o}\right)^{*} \stackrel{\underline{\longrightarrow}}{\longrightarrow} W(\mathfrak{o}), \quad w=\sum_{i=1}^{d} w_{i}\left(\pi^{-m} b_{i}^{\vee}\right) \mapsto \underline{w}=\left(w_{1}, \ldots, w_{d}\right) .
$$

The duality of the two bases means that $w(z)=\underline{z} \cdot \underline{w}:=\sum_{i=1}^{d} z_{i} w_{i}$ for $z \in \mathfrak{g}^{m}$ and $w \in \operatorname{Hom}_{\mathfrak{o}}\left(\mathfrak{g}^{m}, \mathfrak{o}\right)^{*}$.

Lemma 3.3. Let $w \in \operatorname{Hom}_{\mathfrak{o}}\left(\mathfrak{g}^{m}, \mathfrak{o}\right)^{*}$ and let $n \in \mathbb{N}_{0}$. Consider the element $\omega \in \operatorname{Irr}_{n}\left(\mathfrak{g}^{m}\right)$ which is represented by $w$. Then for every $z \in \mathfrak{g}^{m}$ we have

$$
z \in \operatorname{Rad}(\omega) \quad \Longleftrightarrow \quad \underline{z} \cdot \pi^{m} \mathcal{R}(\underline{w}) \equiv_{\mathfrak{p}^{n}} \underline{0} .
$$

Proof. Observe that $x$ belongs to $\operatorname{Rad}(\omega)$ if and only if $\omega\left(\left[x, \pi^{m} b_{i}\right]\right)=0$ for all $i \in$ $\{1, \ldots, d\}$, and express this condition in co-ordinates.

Lemma 3.4. Let $w \in \operatorname{Hom}_{\mathfrak{o}}\left(\mathfrak{g}^{m}, \mathfrak{o}\right)^{*}$ and let $n \in \mathbb{N}_{0}$. Consider the element $\omega \in \operatorname{Irr}_{n}\left(\mathfrak{g}^{m}\right)$ which is represented by $w$. Let $\mathbf{a}:=\nu(\mathcal{R}(\underline{w}))$. Then

$$
\left|\mathfrak{g}^{m}: \operatorname{Rad}(\omega)\right|=q^{2 \sum_{i=1}^{\lfloor d / 2\rfloor}\left(n-m-\min \left\{a_{i}, n-m\right\}\right)} .
$$

Proof. We use Lemma 3.3. If $n \leq m$, then both expressions are equal to 1 . Now suppose that $\tilde{n}:=n-m>0$. Then the claim follows from $q=|\mathfrak{o}: \mathfrak{p}|$ and

$$
\begin{aligned}
\left|\mathfrak{g}^{m}: \operatorname{Rad}(\omega)\right| & =\left|\mathfrak{o}^{2\lfloor d / 2\rfloor}: \bigoplus_{i=1}^{\lfloor d / 2\rfloor}\left(\mathfrak{p}^{\tilde{n}-\min \left\{a_{i}, \tilde{n}\right\}}\right)^{(2)}\right| \\
& =|\mathfrak{o}: \mathfrak{p}|^{2 \sum_{i=1}^{\lfloor d / 2\rfloor}\left(\tilde{n}-\min \left\{a_{i}, \tilde{n}\right\}\right)} .
\end{aligned}
$$

For $\omega \in \widehat{\mathfrak{g}}$ and $n \in \mathbb{N}_{0}$ with $\operatorname{lev}_{\mathfrak{o}}(\omega) \geq n$ we define $\nu_{n}(\omega):=\nu_{n}(\mathcal{R}(\underline{w}))$ where $w \in W(\mathfrak{o})$ is a representative of $\omega$. Clearly this definition does not depend on the particular choice of $w$.

Lemma 3.5. Let $n \in \mathbb{N}_{0}$ with $n \geq m$ and $\mathbf{a} \in \mathbb{N}_{0}^{\lfloor d / 2\rfloor}$. Then

$$
q^{d m} \mathcal{N}_{n-m, \mathbf{a}}^{\mathfrak{o}}=\#\left\{\omega \in \operatorname{Irr}_{n}\left(\mathfrak{g}^{m}\right) \mid \nu_{n-m}(\omega)=\mathbf{a}\right\} .
$$

Proof. Consider the natural projection $\operatorname{Irr}_{n}\left(\mathfrak{g}^{m}\right) \rightarrow \operatorname{Irr}_{n-m}\left(\mathfrak{g}^{m}\right)$. In our parametrisation, this corresponds to the natural projection $W_{n}(\mathfrak{o}) \rightarrow W_{n-m}(\mathfrak{o})$, and each fibre has cardinality $q^{d m}$.

Proof of Proposition 3.1. According to (2.1), (3.3) and the previous three lemmata we have

$$
\begin{aligned}
\zeta_{\mathrm{G}^{m}}(s)= & \sum_{n=0}^{m} \sum_{\omega \in \operatorname{Irr}_{n}\left(\mathfrak{g}^{m}\right)}\left|\mathfrak{g}^{m}: \operatorname{Rad}(\omega)\right|^{-(s+2) / 2} \\
& +\sum_{n=m+1}^{\infty} \sum_{\omega \in \operatorname{Irr}_{n}\left(\mathfrak{g}^{m}\right)}\left|\mathfrak{g}^{m}: \operatorname{Rad}(\omega)\right|^{-(s+2) / 2} \\
= & q^{d m}+\sum_{n=1}^{\infty} \sum_{\mathbf{a} \in \mathbb{N}_{0}^{\lfloor d / 2\rfloor}} q^{d m} \mathcal{N}_{n, \mathbf{a}}^{\mathfrak{o}} q^{-\left(\sum_{i=1}^{\lfloor d / 2\rfloor}\left(n-a_{i}\right)\right)(s+2)} \\
= & q^{d m} \mathcal{P}_{\mathcal{R}, \mathfrak{o}}(s+2) .
\end{aligned}
$$


3.2. Integral formula. As explained in [51, Section 2.2] (in a more general setting where the underlying matrix need not be antisymmetric), the Poincaré series defined in (3.3) can be expressed by a p-adic integral generalising Igusa local zeta functions. We first state the relevant formulae and then explain all the notation. In accordance with [51, Section 2.2], we can write

$$
\mathcal{P}_{\mathcal{R}, \mathfrak{o}}(s)=1+\left(1-q^{-1}\right)^{-1} \mathcal{Z}_{\mathfrak{o}}(-s / 2, \rho s-d-1)
$$

with

$$
z_{\mathfrak{o}}(r, t)=\int_{(x, \mathbf{y}) \in \mathfrak{p} \times W(\mathfrak{o})}|x|_{\mathfrak{p}}^{t} \prod_{j=1}^{\rho} \frac{\left\|F_{j}(\mathbf{y}) \cup F_{j-1}(\mathbf{y}) x^{2}\right\|_{\mathfrak{p}}^{r}}{\left\|F_{j-1}(\mathbf{y})\right\|_{\mathfrak{p}}^{r}} d \mu(x, \mathbf{y}),
$$

where $\mathfrak{p} \times W(\mathfrak{o}) \subseteq \mathfrak{o}^{d+1}$ and the additive Haar measure $\mu$ on $\mathfrak{o}^{d+1}$ is normalised so that $\mu\left(\mathfrak{o}^{d+1}\right)=1$. The individual terms occurring in the formulae (3.4) and (3.5) have the following meaning:

$$
\begin{aligned}
2 \rho & =\max \left\{\operatorname{rk}_{\mathfrak{k}}(\mathcal{R}(\mathbf{y})) \mid \mathbf{y} \in \mathfrak{o}^{d}\right\}, \\
F_{j}(\mathbf{Y}) & =\{f \mid f=f(\mathbf{Y}) \text { a } 2 j \times 2 j \text { minor of } \mathcal{R}(\mathbf{Y})\}, \\
\|F(\mathbf{y})\|_{\mathfrak{p}} & =\max \left\{|f(\mathbf{y})|_{\mathfrak{p}} \mid f \in F\right\} .
\end{aligned}
$$

We note that in accordance with this definition $F_{0}(\mathbf{Y})=\{1\}$, as the empty matrix has determinant 1 .

Remark 3.6. It is worth noting that the sets $F_{j}(\mathbf{Y})$ may be replaced by (smaller) sets of polynomials defining the same polynomial ideals. The ensuing reduction in the number of polynomials may be of relevance for a computational approach toward understanding the associated varieties. Specifically, in the proof of Theorem $\mathbf{E}$ we make use of the fact that one can replace $F_{j}(\mathbf{Y})$ by the set of all principal $2 j \times 2 j$ minors of $\mathcal{R}(\mathbf{Y})$, noting that they are all squares in $\mathfrak{o}[\mathbf{Y}]$. Indeed, an antisymmetric $d \times d$-matrix $M$ over a field has rank less than $2 j$ if and only if all principal $2 j \times 2 j$ minors vanish. This is evident if $M$ is in 'Witt normal form'

$$
M=\left(\begin{array}{ccc}
0 & \mathrm{Id}_{r} & \\
-\mathrm{Id}_{r} & 0 & \\
& & 0
\end{array}\right)
$$

for some $r \in\{0, \ldots,\lfloor d / 2\rfloor\}$. For the general case it suffices to observe that $M$ can be brought into Witt normal form by simultaneous elementary row and column operations, and that the set of matrices with the desired property is closed under these operations. The latter follows from an easy matrix computation, using the fact that antisymmetric matrices have even ranks.

In fact, it is known that every minor of an antisymmetric matrix is a quadratic polynomial in Pfaffians (i.e. square roots of principal minors); cf. [7, 23.

Equations (3.4) and (3.5) show that the Poincaré series we are interested in is essentially a generalised Igusa local zeta function, which can therefore be studied by a number of known tools; cf. [50] and Section 4. The main ingredient in deriving (3.4) is that the numbers $\mathcal{N}_{n, \mathbf{a}}^{o}$ which enter into the definition of the Poincare series encode precisely the measures $\mu(S)$ of subsets $S \subseteq \mathfrak{p} \times W(\mathfrak{o})$ on which the integrand in (3.5) is constant. Indeed, a short indication of how to derive (3.4) is as follows. Temporarily, set

$$
P(x, \mathbf{y}):=\prod_{j=1}^{\lfloor d / 2\rfloor} \frac{\left\|F_{j}(\mathbf{y}) \cup F_{j-1}(\mathbf{y}) x^{2}\right\|_{\mathfrak{p}}}{\left\|F_{j-1}(\mathbf{y})\right\|_{\mathfrak{p}}}
$$


for convenience of notation. Then we have

$$
\begin{aligned}
& \mathcal{P}_{\mathcal{R}, \mathfrak{o}}(s)-1=\sum_{n \in \mathbb{N}} \sum_{\mathbf{a} \in \mathbb{N}_{0}^{\lfloor d / 2\rfloor}} \mathcal{N}_{n, \mathbf{a}}^{\mathfrak{o}} q^{-\left(\sum_{i=1}^{\lfloor d / 2\rfloor}\left(n-a_{i}\right)\right) s} \\
& =\sum_{n \in \mathbb{N}} \sum_{\substack{\mathbf{y} \in W_{n}(\mathfrak{o}) \\
\mathbf{a}:=\nu(\overline{\mathbf{y}})}} q^{-\left(\sum_{i=1}^{\lfloor d / 2\rfloor}\left(n-a_{i}\right)\right) s} \\
& =\int_{x \in \mathfrak{p}} \frac{1}{\left(1-q^{-1}\right)|x|_{\mathfrak{p}}} \int_{\mathbf{y} \in W(\mathfrak{o})}|x|_{\mathfrak{p}}^{-d}\left(|x|_{\mathfrak{p}}^{-2\lfloor d / 2\rfloor} P(x, \mathbf{y})\right)^{-s / 2} d \mu(x, \mathbf{y}) \\
& =\left(1-q^{-1}\right)^{-1} \int_{(x, \mathbf{y}) \in \mathfrak{p} \times W(\mathfrak{o})}|x|_{\mathfrak{p}}^{\lfloor d / 2\rfloor s-d-1} P(x, \mathbf{y})^{-s / 2} d \mu(x, \mathbf{y}) .
\end{aligned}
$$

The penultimate equality can be justified as follows. The region of integration $\mathfrak{p}$ for $x$ is divided into disjoint subsets $X_{n}:=\mathfrak{p}^{n} \backslash \mathfrak{p}^{n+1}=\left\{\left.x \in \mathfrak{o}|| x\right|_{\mathfrak{p}}=q^{-n}\right\}, n \in \mathbb{N}$, and the singleton $\{0\}$, which is negligible as it has measure 0 . Fix $n \in \mathbb{N}$. Evaluating the integral on $X_{n}$, the term $\left(\left(1-q^{-1}\right)|x|_{\mathfrak{p}}\right)^{-1}$ cancels with the measure of the set $X_{n}$. For any $x \in X_{n}$ and $\mathbf{y} \in W(\mathfrak{o})$, the term $|x|_{\mathfrak{p}}^{-2\lfloor d / 2\rfloor} P(x, \mathbf{y})$ is equal to $q^{2 \sum_{i}\left(n-\min \left\{a_{i}, n\right\}\right)}$, where $\mathbf{a}=\nu_{n}(\mathbf{y})$. Finally, the term $|x|_{\mathfrak{p}}^{-d}$ is the size of the set $\left(\mathfrak{o} / \mathfrak{p}^{n}\right)^{(d)}$ and compensates for the fact that we use integration over $\mathbf{y} \in W(\mathfrak{o})$ to represent summation over $\overline{\mathbf{y}} \in W_{n}(\mathfrak{o})$. A short thought reveals that the expression $\lfloor d / 2\rfloor$ in the exponent of $|x|_{\mathfrak{p}}$ may be replaced by the invariant $\rho$ without altering the value of the integral.

We collect the results of this section as a formal corollary to Proposition 3.1 .

Corollary 3.7. Let $\mathfrak{g}$ be as defined at the beginning of Section 3. Then for all $m \in \mathbb{N}_{0}$ which are permissible for $\mathfrak{g}$ we have

$$
\zeta_{\mathrm{G}^{m}}(s)=q^{d m}\left(1+\left(1-q^{-1}\right)^{-1} z_{\mathfrak{o}}(-s / 2-1, \rho(s+2)-d-1)\right),
$$

where $Z_{\mathfrak{o}}(r, t)$ is the $\mathfrak{p}$-adic integral defined in (3.5).

\section{Functional Equations And poles}

In this section we prove Theorems $\mathrm{A}$ and $\mathrm{B}$. As in Section 1.2 we denote by $\Lambda$ a Lie lattice over the ring of integers $\mathcal{O}$ in a number field $k$. We assume that $\Lambda$ is rationally perfect, i.e. that $k \otimes_{\mathfrak{o}} \Lambda$ is a perfect $k$-Lie algebra, of $k$-dimension $d$. Given a nonarchimedean place $v$ of $k$ above the rational prime $p$, we denote by $\mathfrak{o}=\mathcal{O}_{v}$ the ring of integers of the completion $k_{v}$ of $k$ at $v$, with residue field cardinality $q_{v}$. Given a finite extension $\mathfrak{O}$ of $\mathfrak{o}$, with maximal ideal $\mathfrak{P}$, we define the $\mathfrak{O}$-Lie lattice $\mathfrak{g}(\mathfrak{O}):=\mathfrak{O} \otimes_{\mathcal{O}} \Lambda$. We denote by $e=e(\mathfrak{O}, \mathfrak{o})$ the ramification index and by $f=f(\mathfrak{O}, \mathfrak{o})$ the degree of inertia of the given extension. For every $m \in \mathbb{N}_{0}$ which is permissible for $\mathfrak{g}(\mathfrak{O}$ ) (cf. Definition 2.2) the principal congruence sublattice $\mathfrak{g}^{m}(\mathfrak{O}):=\mathfrak{P}^{m} \mathfrak{g}(\mathfrak{O})$ corresponds to a potent and saturable FAb pro- $p$ group $G^{m}(\mathfrak{O})=\exp \left(\mathfrak{g}^{m}(\mathfrak{O})\right)$. We assume throughout this section that $m$ is permissible for the relevant Lie lattices.

The $\mathfrak{p}$-adic formalism developed in Section 3 applies to the representation zeta functions $\zeta_{\mathrm{G}^{m}(\mathfrak{O})}(s)$. However, whereas the setup in Section 3 is a local one, the results in the current section exploit the fact that the groups $\mathrm{G}^{m}(\mathfrak{O})$ are all derived from one global object defined over $\mathcal{O}$, viz. the Lie lattice $\Lambda$. This fact has an important consequence for the integrands of the $\mathfrak{p}$-adic integrals which we set up to describe the relevant representation zeta functions. Indeed, with the integral $z_{\mathfrak{O}}(r, t)$ defined as in (3.5) we have, by Corollary 3.7 ,

$$
\zeta_{\mathrm{G}^{m}(\mathfrak{O})}(s)=q^{f d m}\left(1+\left(1-q^{-f}\right)^{-1} z_{\mathfrak{D}}(-s / 2-1, \rho(s+2)-d-1)\right) .
$$


Recall that the parameter $\rho$ was defined in (3.6). The polynomials $F_{j}(\mathbf{Y})$ occurring in the integrand of $z_{\mathfrak{O}}(r, t)$ are defined over $\mathcal{O}$, as they are minors of the commutator matrix $\mathcal{R}(\mathbf{Y})$ of $\Lambda$ with respect to a fixed o-basis. Thus, essentially only the integral's domain of integration varies with the ring $\mathfrak{O}$.

4.1. We prove Theorems $\mathrm{A}$ and $\mathrm{B}$ by describing explicit formulae for the relevant $\mathfrak{p}$-adic integrals, generalising expressions given in [51]. We recall from there what we require to make the present paper reasonably self-contained, and adapt the notation from [51] to the conventions of the present context.

4.1.1. Fix $n, b, l \in \mathbb{N}$, let $I \subseteq\{1, \ldots, n-1\}$, and $\mathcal{W}(\mathfrak{o}) \subseteq \mathfrak{o}^{b}$ be a union of cosets modulo $\mathfrak{p}^{(b)}$. Section 2 of [51] develops, in a more general context, formulae for multivariate $\mathfrak{p}$-adic integrals of the form

$$
Z_{\mathcal{W}(\mathfrak{o}), I}(\mathbf{s}):=\int_{\mathfrak{p}(|I|) \times \mathcal{W}(\mathfrak{o})} \prod_{\kappa=1}^{l}\left\|\bigcup_{\iota \in J_{\kappa}}\left(\prod_{i \in I} x_{i}^{e_{i \kappa \iota}}\right) F_{\kappa \iota}(\mathbf{y})\right\|_{\mathfrak{p}}^{s_{\kappa}} d \mu(\mathbf{x}, \mathbf{y})
$$

with a view toward proving functional equations under 'inversion of the parameter $q$ ' for generating functions which are expressible in terms of such integrals. Here $\mathbf{s}=\left(s_{1}, \ldots, s_{l}\right)$ is a vector of complex variables, the sets $J_{\kappa}, \kappa \in\{1, \ldots, l\}$, are finite index sets, $\mathbf{x}=\left(x_{1}, \ldots, x_{n-1}\right)$ and $\mathbf{y}=\left(y_{1}, \ldots, y_{b}\right)$ are independent variables, $e_{i \kappa \iota}$ are nonnegative integers and $F_{\kappa \iota}(\mathbf{Y})$ are finite sets of polynomials over $\mathcal{O}$.

A general idea in the study of a $\mathfrak{p}$-adic integral like (4.1) is to study its transformation under a (log-)principalisation of the ideals defined by the polynomials occurring in the integrand. A principalisation of ideals not only provides an embedded resolution of singularities for the relevant varieties, but also renders them locally principal. The existence of principalisations of ideals in characteristic 0 lies as deep as Hironaka's theorem on resolutions of singularities in characteristic 0; cf. [50] and references therein. The domain of the transformed integral may be partitioned into finitely many (p-adically open) co-ordinate neighbourhoods on which the evaluation of the integral is reduced to the computation of zeta functions enumerating integral points of systems of polyhedral cones. The multiplicities to which each of these systems occur are given by the numbers of rational points over finite fields of constructible algebraic sets. Explicit formulae resulting from this kind of analysis yield, in many cases, supersets of the set of real parts of the poles of the integral, and can be used to explain phenomena like local functional equations. In the present setting, we obtain principalisations over various local fields from a fixed principalisation defined over $k$ by base extension. This allows us to derive formulae which are 'uniform' both with respect to the variation of the place and under ring extensions.

Concretely, we fix a principalisation $(Y, h)$ over $k$ of the ideal $\mathcal{J}:=\prod_{\kappa, \iota}\left(F_{\kappa \iota}(\mathbf{Y})\right) \subseteq$ $k[\mathbf{Y}]$, with numerical data $\left(N_{t \kappa \iota}, \nu_{t}\right)_{t \kappa \iota}$, where $t \in T, \kappa \in\{1, \ldots, l\}, \iota \in J_{\kappa}$. Here $T$ is a finite index set for the irreducible components $E_{t}$ of the pre-image under $h$ of the variety defined by J. For $t, \kappa, \iota$ as above, the number $\nu_{t}-1$ denotes the multiplicity of $E_{t}$ in the divisor of $h^{*}(d \mu(\mathbf{y}))$, and $N_{t \kappa \iota}$ denotes the multiplicity of $E_{t}$ in the pre-image under $h$ of the variety defined by the ideal $\mathcal{J}_{\kappa \iota}:=\left(F_{\kappa \iota}(\mathbf{Y})\right) \subseteq k[\mathbf{Y}]$; cf. [51, Section 2.1]. Theorem 2.2 and Corollary 2.1 of [51] provide explicit formulae for the p-adic integrals $Z_{\mathcal{W}(\mathfrak{o}), I}(\mathbf{s})$ under additional invariance and regularity conditions - specified just before [51, Theorem 2.2] - on the ideals $\mathcal{J}_{\kappa \iota}$ and on $(Y, h)$. In our applications to representation zeta functions, the invariance conditions will always be satisfied for all places of $k$, and 
are henceforth assumed to hold. They include, in particular, the condition that $b=n^{2}$. The regularity conditions comprise the condition that $\left(Y_{\mathfrak{k}}, h_{\mathfrak{k}}\right)$, the principalisation over $\mathfrak{k}$ obtained from $(Y, h)$ by base extension, has good reduction modulo $\mathfrak{p}$, and that none of the ideals $\mathcal{J}_{\kappa \iota}$ is zero modulo $\mathfrak{p}$. We denote by $S$ the finite set of places of $k$ for which these conditions fail to hold.

The general strategy outlined above is illustrated in the current context by [51, Theorem 2.1] which states that, under the stated conditions,

$$
Z_{\mathcal{W}(\mathfrak{o}), I}(\mathbf{s})=\left(1-q^{-1}\right)^{|I|} q^{-n^{2}} \sum_{U \subseteq T} c_{U}(q)(q-1)^{|U|} \Xi_{U, I}(q, \mathbf{s}) .
$$

Here, for any $U \subseteq T$, the coefficient

$$
c_{U}(q):=c_{U, \mathcal{W}(\mathfrak{o})}(q)=\mid\left\{a \in Y(\mathfrak{o} / \mathfrak{p}) \mid\left(a \in E_{u}(\mathfrak{o} / \mathfrak{p}) \Leftrightarrow u \in U\right) \text { and } \overline{h_{\mathfrak{k}}}(a) \in \overline{\mathcal{W}(\mathfrak{o})}\right\} \mid,
$$

where ${ }^{-}$denotes reduction modulo $\mathfrak{p}$, is the number of $\mathbb{F}_{q}$-rational points of the (reduction modulo $\mathfrak{p}$ of the) constructible set determined by the boolean combination of the varieties $E_{u}$ prescribed by $U$. Furthermore we set, for $U \subseteq T, \mathbf{m} \in \mathbb{N}^{U}, \mathbf{n} \in \mathbb{N}^{I}$,

$$
\begin{aligned}
L(\mathbf{m}, \mathbf{n}) & :=-\sum_{i \in I} n_{i}-\sum_{u \in U} \nu_{u} m_{u}, \\
L_{\kappa \iota}(\mathbf{m}, \mathbf{n}) & :=\sum_{i \in I} e_{i \kappa \iota} n_{i}+\sum_{u \in U} N_{u \kappa \iota} m_{u}, \quad \text { for } \kappa \in\{1, \ldots, l\}, \iota \in J_{\kappa},
\end{aligned}
$$

and

$$
\Xi_{U, I}(q, \mathbf{s}):=\sum_{\mathbf{m} \in \mathbb{N}^{U}, \mathbf{n} \in \mathbb{N}^{I}} q^{L(\mathbf{m}, \mathbf{n})-\sum_{\kappa=1}^{l} s_{\kappa} \min \left\{L_{\kappa \iota}(\mathbf{m}, \mathbf{n}) \mid \iota \in J_{\kappa}\right\}} .
$$

In order to analyse the functions $\Xi_{U, I}(q, \mathbf{s})$ for fixed $U$ and $I$, we may cover the open orthant $\mathbb{R}_{>0}^{|U|+|I|}$ by a family $\mathfrak{C}$ of (not necessarily disjoint) rational polyhedral cones such that, on each cone $\mathcal{C}$ in $\mathfrak{C}$, the value of each of the 'min'-terms in (4.3) is attained by a single linear functional $L_{\kappa \iota}$. Indeed, for $g=\left(g_{\kappa}\right) \in \prod_{\kappa=1}^{l} J_{\kappa}$, let $\mathcal{C}_{g}$ be the cone in $\mathbb{R}_{>0}^{|U|+|I|}$ defined by the condition

$$
\forall \kappa \in\{1, \ldots, l\} \forall \iota \in J_{\kappa}: L_{\kappa g_{\kappa}} \leq L_{\kappa \iota} .
$$

Then the family $\mathfrak{C}:=\left(\mathfrak{C}_{g}\right)_{g \in \prod_{\kappa} J_{\kappa}}$ of closed polyhedral cones clearly has the desired property. For each $g \in \prod_{\kappa} J_{\kappa}$, the restriction of the summation in (4.3) to $\mathcal{C}_{g} \cap \mathbb{N}^{|U|+|I|}$ may be interpreted in terms of a multivariate Hilbert series associated to the monoid of solutions of a homogeneous system $A_{g} \mathbf{x}=\mathbf{0}$ of linear diophantine equations (cf. 48, I.3]). Here $A_{g}$ is a matrix with integer coefficients. It is well-known that these Hilbert series are rational functions in variables $\xi_{1}, \ldots, \xi_{|U|+|I|}$, say, and that their denominators are finite products of the form $\prod_{i}\left(1-\prod_{j=1}^{|U|+|I|} \xi_{j}^{n_{i j}}\right)$ for integers $n_{i j}$ depending on $\mathcal{C}_{g}$. A formula for the restriction of the summation in (4.3) to $\mathcal{C}_{g} \cap \mathbb{N}^{|U|+|I|}$ can be obtained by substituting for these variables suitable monomials in $q$ and $q^{-s_{\kappa}}, \kappa \in\{1, \ldots, l\}$.

In the case that $v \notin S,[51$, Corollary 2.1] translates (4.2) into an explicit formula for the normalised integrals

$$
\widetilde{Z_{\mathcal{W}(\mathfrak{o}), I}}(\mathbf{s}):=Z_{\mathcal{W}(\mathfrak{o}), I}(\mathbf{s})\left(\left(1-q^{-1}\right)^{|I|} \prod_{i=1}^{n}\left(1-q^{-i}\right)\right)^{-1} .
$$

More precisely, we have that

$$
\widetilde{Z_{\mathcal{W}(\mathfrak{o}), I}}(\mathbf{s})=\left(\prod_{i=1}^{n} \frac{q-1}{q^{i}-1}\right) \sum_{U \subseteq T} b_{U}(q) \sum_{V \subseteq U}(-1)^{|U \backslash V|}(q-1)^{|V|} \Xi_{V, I}(q, \mathbf{s}),
$$


where, for each $U$, we denote by $b_{U}(q)$ the number of $\mathbb{F}_{q}$-rational points of the reduction modulo $\mathfrak{p}$ of the smooth projective algebraic variety $E_{U}:=\cap_{u \in U} E_{u}$. These are related to the numbers $c_{U, \mathcal{W}(\mathfrak{o})}(q)$ in a simple way by the inclusion-exclusion principle. Crucially for our application to the proof of Theorem [A], Corollary 2.4 of [51] establishes, for all $i \in\{1, \ldots, n-1\}$, a functional equation of the form

$$
\begin{aligned}
\left(\widetilde{Z_{\mathcal{W}(\mathfrak{o}), \varnothing}}(\mathbf{s})+\left(1-q^{-n}\right) \widetilde{Z_{\mathcal{W}(\mathfrak{o}),\{i\}}}(\mathbf{s})\right) & \left.\right|_{\substack{q \rightarrow q^{-1} \\
\lambda_{i} \rightarrow \lambda_{i}^{-1}}}= \\
& q^{-n}\left(\widetilde{Z_{\mathcal{W}(\mathfrak{o}), \varnothing}}(\mathbf{s})+\left(1-q^{-n}\right) \widetilde{Z_{\mathcal{W}(\mathfrak{o}),\{i\}}}(\mathbf{s})\right) .
\end{aligned}
$$

Definitions of the terms $\lambda_{i}$ and a precise explanation of the operation $q \rightarrow q^{-1}, \lambda_{i} \rightarrow \lambda_{i}^{-1}$ are given in Section 4.2 ,

4.1.2. Our aim for the remainder of this section is fourfold: in Section 4.1.3 we explain how the formalism developed in Section 4.1.1 applies to the study of representation zeta functions of groups of the form $G^{m}(\mathfrak{O})$ featuring in Theorems $\mathrm{A}$ and $\mathrm{B}$. In Section 4.2 we show that, in the case $v \notin S$, the formula (4.4) and the functional equations (4.5) hold uniformly for all extensions $\mathfrak{O} \mid \mathfrak{o}$ (possibly ramified), upon substitution of $q^{f}$ for $q$, where $f=f(\mathfrak{O}, \mathfrak{o})$; the precise meaning of this substitution is explained in Section 4.2. This uniformity phenomenon under field extensions is in analogy with the situation for Igusa local zeta functions; cf. [13, Theorem 3]. In Section 4.3 we give a formula for $Z_{\mathcal{W}(\mathfrak{o}), I}(\mathbf{s})$, generalising (4.2), which is also valid for $v \in S$. The main idea there is to write the integral as a sum over suitably chosen $\mathfrak{p}$-adic co-ordinate neighbourhoods, indexed by the cosets of $Y(\mathfrak{o})$ modulo $\mathfrak{p}^{N}$ for some $N \in \mathbb{N}$, on which the integrand is 'monomial'. This generalises the case of good reduction, where $N=1$ is sufficient. We use the Greenberg transform of level $N$ to identify the points of $Y\left(\mathfrak{o} / \mathfrak{p}^{N}\right)$ for which the (p-adically) 'local' integrals are identical with rational points of constructible subsets of a variety defined over $\mathbb{F}_{q}$. (In explicit co-ordinates this amounts to a description in terms of Witt vectors.) In Section 4.3.1 we exploit the functoriality of the Greenberg transform to obtain a formula which holds uniformly for all unramified extensions of $\mathfrak{o}$. In Section 4.3 .2 we study totally ramified extensions $\mathfrak{D} \mid \mathfrak{o}$. Dealing with these special cases of ramification is sufficient for our purposes, as every extension $\mathfrak{O} \mid \mathfrak{o}$ has a unique maximal unramified subextension $\mathfrak{O}^{\text {ur }} \mid \mathfrak{o}$ such that $\mathfrak{O}^{\text {ur }} \mid \mathfrak{O}$ is totally ramified. Section 4.4 contains a key result on poles of zeta functions of cones. The proofs of Theorems $\mathrm{A}$ and B are given in Section 4.5.

4.1.3. The link between the formulae given in Section 4.1.1 and representation zeta functions of groups is as follows. How, in general, Poincaré series enumerating elementary divisors of matrices of forms may be expressed in terms of integrals of the form (4.1) is explained in [51, Section 2.2]. In this application of the general p-adic integration framework developed in [51, the set $\mathcal{W}(\mathfrak{o})$ is $\mathrm{GL}_{d}(\mathfrak{o})$, but in fact the integrand only depends on the entries in the first rows, say, of matrices $\mathbf{y} \in \mathrm{GL}_{d}(\mathfrak{o})$. This connects $\mathcal{W}(\mathfrak{o})$ with the set $W(\mathfrak{o})=\left(\mathfrak{o}^{d}\right)^{*}$ introduced in Section 3.1 of the present paper.

In our context, setting $n:=d, b:=d^{2}$ and $\mathcal{W}(\mathfrak{O}):=\mathrm{GL}_{d}(\mathfrak{O})$, it is easy to deduce from (3.5) that, for suitable data of $l, J_{\kappa}, F_{\kappa l}(\mathbf{y})$ in (4.1), and vectors $\mathbf{a}, \mathbf{b} \in \mathbb{Z}^{l}, I \in$ $\{\varnothing,\{1\}\}$,

$$
\begin{aligned}
& \widetilde{Z_{\mathcal{W}(\mathfrak{O}), \varnothing}}(\mathbf{a} s+\mathbf{b})=1, \\
& \left(1-q^{-f d}\right) \widetilde{Z_{\mathcal{W}(\mathfrak{O}),\{1\}}}(\mathbf{a} s+\mathbf{b})=\left(1-q^{-f}\right)^{-1} z_{\mathfrak{O}}(-s / 2-1, \rho(s+2)-d-1) \text {. }
\end{aligned}
$$


Here we write $\mathbf{a} s+\mathbf{b}$ for $\left(a_{1} s+b_{1}, \ldots, a_{l} s+b_{l}\right)$. Invoking Corollary 3.7 we thus have

$$
\begin{aligned}
\zeta_{\mathrm{G}^{m}(\mathfrak{D})}(s) & =q^{f d m}\left(1+\left(1-q^{-f}\right)^{-1} \mathcal{Z}_{\mathfrak{D}}(-s / 2-1, \rho(s+2)-d-1)\right) \\
& =q^{f d m}\left(\widetilde{Z_{\mathcal{W}(\mathfrak{O}), \varnothing}}(\mathbf{a} s+\mathbf{b})+\left(1-q^{-f d}\right) \widetilde{Z_{\mathcal{W}(\mathfrak{O}),\{1\}}}(\mathbf{a} s+\mathbf{b})\right) .
\end{aligned}
$$

The sets $F_{\kappa \iota}(\mathbf{Y})$ featuring in the integrand of the integral $\mathcal{Z}_{\mathfrak{O}}(r, t)$ are given by sets of minors of the commutator matrix $\mathcal{R}(\mathbf{Y})$ associated to the lattice $\Lambda$; cf. Section 3.2 .

4.2. Places with good reduction. Recall from Section 4.1 .1 that $(Y, h)$ is a principalisation of ideals for the ideal $\mathcal{J} \subseteq k[\mathbf{Y}]$ defined over the number field $k$. We obtain, by base extension, a principalisation $\left(Y_{\mathfrak{K}}, h_{\mathfrak{K}}\right)$ over every extension $\mathfrak{K} \mid \mathfrak{k}$, where $\mathfrak{k}=k_{v}$ for a non-archimedean place $v$ of $k$. Throughout Section 4.2 we assume that $v \notin S$. For such $v$, all of the principalisations $\left(Y_{\mathfrak{K}}, h_{\mathfrak{K}}\right)$ have good reduction modulo $\mathfrak{P}$; cf. [11, Proposition 2.3 and Theorem 2.4]. The formulae in [51, Theorem 2.2 and Corollary 2.1] extend to 'uniform' formulae for

$$
\widetilde{Z_{\mathcal{W}(\mathfrak{O}), I}}(\mathbf{s}):=Z_{\mathcal{W}(\mathfrak{O}), I}(\mathbf{s})\left(\left(1-q^{-f}\right)^{|I|} \prod_{i=1}^{n}\left(1-q^{-i f}\right)\right)^{-1}
$$

where $\mathfrak{O}$ is any finite extension of $\mathfrak{o}$ with $f=f(\mathfrak{O}, \mathfrak{o})$ : as in [13, Theorem 3] one can argue that an expression for the integrals $\widetilde{Z_{\mathcal{W}(\mathfrak{D}), I}(s)}$ is obtained by replacing $q$ by $q^{f}$ on the right hand side of (4.4). This description also allows us to explain functional equations like (4.5). Indeed, the numbers $b_{U}\left(q^{f}\right)$ in (4.4) may, by the Weil conjectures, be written as alternating sums of powers of Frobenius eigenvalues, i.e.

$$
b_{U}\left(q^{f}\right)=\sum_{i=0}^{2\left(\left(\begin{array}{c}
n \\
2
\end{array}\right)-|U|\right)}(-1)^{i} \sum_{j=1}^{t_{U, i}} \alpha_{U, i, j}^{f}
$$

for suitable nonnegative integers $t_{U, i}$ and non-zero complex numbers $\alpha_{U, i, j}$; cf. [51, Section 2.1] and [13, Section 2]. The numbers $\alpha_{U, i, j}$ have the property that

$$
b_{U}\left(q^{-f}\right):=\sum_{i=0}^{2\left(\left(\begin{array}{c}
n \\
2
\end{array}\right)-|U|\right)}(-1)^{i} \sum_{j=1}^{t_{U, i}} \alpha_{U, i, j}^{-f}=q^{-f\left(\left(\begin{array}{l}
d \\
2
\end{array}\right)-|U|\right)} b_{U}\left(q^{f}\right) .
$$

We note that the numbers $\alpha_{U, i, j}$ are in fact algebraic integers, and relabel them to $\lambda_{1}=\lambda_{1}(v), \ldots, \lambda_{r}=\lambda_{r}(v)$. The functions $\Xi_{U, I}(q, \mathbf{s})$ are rational functions in monomials in $q$ and $q^{-s_{\kappa}}, \kappa \in\{1, \ldots, l\}$, and the effect of inverting these terms is clear. The proof of [51, Corollary 2.4] may thus be extended to show that, for $i \in\{1, \ldots, n-1\}$,

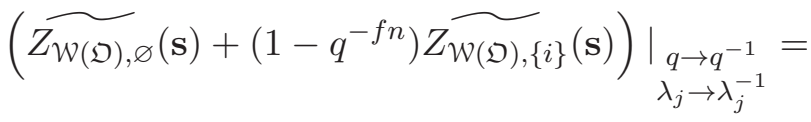

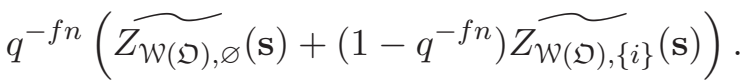

4.3. Places with bad reduction. We now drop the assumption that $v \notin S$. For every non-archimedean place $v$ of $k$ there exists $N \in \mathbb{N}$, such that $Y(\mathfrak{o})$ can be covered disjointly by cosets modulo $\mathfrak{p}^{N}$, which are homeomorphic to $\left(\mathfrak{p}^{N}\right)^{(b)}$ and indexed by the elements of $Y\left(\mathfrak{o} / \mathfrak{p}^{N}\right)$, on which the terms in the integrand are monomial, possibly allowing for a factor with locally constant $\mathfrak{p}$-adic valuation, and on which the Haar measure transforms in a similar way. More precisely, we can choose $N \in \mathbb{N}$ such that, on the cosets $\left\{\mathbf{y} \in Y(\mathfrak{o}) \mid \mathbf{y} \equiv \mathbf{a} \bmod \mathfrak{p}^{N}\right\}$, indexed by the elements $\mathbf{a} \in Y\left(\mathfrak{o} / \mathfrak{p}^{N}\right)$, the 
following holds: there exist $U=U(\mathbf{a}) \subseteq T, j=j(\mathbf{a}) \in \mathbb{N}_{0}$ and, for $\kappa \in\{1, \ldots, l\}, \iota \in J_{\kappa}$, integers $d_{\kappa \iota}=d_{\kappa \iota}(\mathbf{a}) \in \mathbb{N}_{0}$ such that

$$
\left\|F_{\kappa \iota} \circ h\right\|_{\mathfrak{p}}=q^{-d_{\kappa \iota}} \prod_{u \in U}\left|\gamma_{u}\right|_{\mathfrak{p}}^{N_{u \kappa \iota}} \quad \text { and } \quad h^{*}(d \mu(\mathbf{y}))=q^{-j} \prod_{u \in U}\left|\gamma_{u}\right|_{\mathfrak{p}}^{\nu_{u}-1} d \mu(\boldsymbol{\gamma}) .
$$

for co-ordinate functions $\gamma_{u}, u \in U$. This follows as $\left(Y_{\mathfrak{k}}, h_{\mathfrak{k}}\right)$ still yields a principalisation of ideals for the $\mathfrak{k}$-manifold defined by $\mathcal{J}$. In fact, we may choose $N$ such that the above properties hold uniformly for all unramified extensions of $\mathfrak{o}$; cf. [41, Theorem 2]. In the case that $v \notin S$, one may choose $N=1$ and all of the integers $j(\mathbf{a}), d_{\kappa \iota}(\mathbf{a})$ are zero for all $\mathbf{a} \in Y(\mathfrak{o} / \mathfrak{p})$. As the set $S$ is finite, we may thus choose $N$ which is good for all places $v$ of $k$. For given $U \subseteq T,\left(d_{\kappa \iota}\right) \in \mathbb{N}_{0}^{\prod_{\kappa} J_{\kappa}}$ and $I \subseteq\{1, \ldots, n-1\}$ we set

$$
\Xi_{U,\left(d_{\kappa \iota}\right), I}^{N}(q, \mathbf{s}):=\sum_{\mathbf{m} \in \mathbb{N}_{\geq N}^{U}, \mathbf{n} \in \mathbb{N}^{I}} q^{L(\mathbf{m}, \mathbf{n})-\sum_{\kappa=1}^{l} s_{\kappa} \min \left\{L_{\kappa \iota}(\mathbf{m}, \mathbf{n})-d_{\kappa \iota} \mid \iota \in J_{\kappa}\right\}} .
$$

Proposition 4.1. For $I \subseteq\{1, \ldots, n-1\}$ we have

$$
Z_{\mathcal{W}(\mathfrak{o}), I}(\mathbf{s})=\frac{\left(1-q^{-1}\right)^{|I|}}{q^{N n^{2}}} \sum_{\substack{\mathbf{a} \in Y\left(\mathfrak{o} / \mathfrak{p}^{N}\right) \\ h_{\mathfrak{k}}(\mathbf{a}) \in \mathcal{W}\left(\mathfrak{o} / \mathfrak{p}^{N}\right)}}\left(q^{N}-q^{N-1}\right)^{|U(\mathbf{a})|} q^{-j(\mathbf{a})} \Xi_{U(\mathbf{a}),\left(d_{\kappa \iota}(\mathbf{a})\right), I}^{N}(q, \mathbf{s}),
$$

where - denotes reduction modulo $\mathfrak{p}^{N}$.

Note that (4.9) generalises (4.2). The proof of Proposition 4.1] is analogous to the proof of [51, Theorem 2.1].

We may rewrite (4.9) by collecting the points $\mathbf{a} \in Y\left(\mathfrak{o} / \mathfrak{p}^{N}\right)$ for which the summands coincide. More precisely we consider, for $U \subseteq T, j \in \mathbb{N}_{0},\left(d_{\kappa \iota}\right) \in \mathbb{N}_{0}^{\prod_{\kappa} J_{\kappa}}$, the sets

$$
\begin{aligned}
& C_{U, j,\left(d_{\kappa \iota}\right)}\left(\mathfrak{o} / \mathfrak{p}^{N}\right):=C_{U, j,\left(d_{\kappa \iota}\right), \mathcal{W}(\mathfrak{o})}\left(\mathfrak{o} / \mathfrak{p}^{N}\right):= \\
& \quad\left\{\mathbf{a} \in Y\left(\mathfrak{o} / \mathfrak{p}^{N}\right) \mid U(\mathbf{a})=U, j(\mathbf{a})=j,\left(d_{\kappa \iota}(\mathbf{a})\right)=\left(d_{\kappa \iota}\right), \overline{h_{\mathfrak{k}}}(\mathbf{a}) \in \mathcal{W}\left(\mathfrak{o} / \mathfrak{p}^{N}\right)\right\} .
\end{aligned}
$$

By the theory of the Greenberg transform (see, e.g., [21]), there exists a variety $y_{N}$ over $\mathbb{F}_{q}$ such that

$$
Y\left(\mathfrak{o} / \mathfrak{p}^{N}\right)=y_{N}\left(\mathbb{F}_{q}\right) .
$$

The sets $C_{U, j,\left(d_{\kappa \iota}\right)}\left(\mathfrak{o} / \mathfrak{p}^{N}\right)$ correspond to the $\mathbb{F}_{q^{-}}$-rational points of constructible subsets $\mathcal{C}_{U, j,\left(d_{\kappa \iota}\right)}$ of $y_{N}$ defined over $\mathbb{F}_{q}$, and

$$
c_{U, j,\left(d_{\kappa \iota}\right)}(q):=\left|\mathcal{C}_{U, j,\left(d_{\kappa \iota}\right)}\left(\mathbb{F}_{q}\right)\right|=\left|C_{U, j,\left(d_{\kappa \iota}\right)}\left(\mathfrak{o} / \mathfrak{p}^{N}\right)\right| .
$$

Thus we obtain

Corollary 4.2. For $I \subseteq\{1, \ldots, n-1\}$ we have

$$
Z_{\mathcal{W}(\mathfrak{o}), I}(\mathbf{s})=\frac{\left(1-q^{-1}\right)^{|I|}}{q^{N n^{2}}} \sum_{U, j,\left(d_{\kappa \iota}\right)} c_{U, j,\left(d_{\kappa \iota}\right)}(q)\left(q^{N}-q^{N-1}\right)^{|U|} q^{-j} \Xi_{U,\left(d_{\kappa \iota}\right), I}^{N}(q, \mathbf{s}) .
$$

This generalises the formula given in [51, Theorem 2.1]. Following arguments analogous to those in [51, Theorem 2.2], we obtain formulae for the normalised integrals $\widetilde{Z_{\mathcal{W}(\mathfrak{o}), I}}(\mathbf{s})$ and thus, via (4.6) and Corollary 3.7, formulae for the respective representation zeta functions.

Remark 4.3. We do not expect in general an analogue of [51, Corollary 2.1] to hold, as it is not clear how the numbers $c_{U, j,\left(d_{\kappa \iota}\right)}(q)$ may be expressed in terms of the numbers of rational points of smooth projective varieties. Local functional equations may therefore not hold for non-archimedean places $v \in S$. This phenomenon is well-known in the 
theory of Igusa local zeta functions and is, in our context, illustrated by formulae for the congruence subgroups of $\mathrm{SL}_{3}(\mathfrak{o})$ in the case of residue field characteristic 3 ; cf. [4].

4.3.1. Unramified extensions. Consider now extensions $\mathfrak{O} \mid \mathfrak{o}$ with $e(\mathfrak{O}, \mathfrak{o})=1$ and $f=$ $f(\mathfrak{O}, \mathfrak{o})$ arbitrary. Recall from the beginning of Section 4.3 that we may choose $N$ such that we can write $Z_{\mathcal{W}(\mathcal{O}), I}(\mathbf{s})$ as a sum over cosets modulo $\mathfrak{P}^{N}$, with the same 'local numerical data' $\left(U(\mathbf{a}), j(\mathbf{a}),\left(d_{\kappa \iota}(\mathbf{a})\right)\right)$ as in the case $\mathfrak{O}=\mathfrak{o}$, uniformly for all $f$. By functoriality of the Greenberg transform we have

$$
Y\left(\mathfrak{O} / \mathfrak{P}^{N}\right)=y_{N}\left(\mathbb{F}_{q^{f}}\right)
$$

and the sets $C_{U, j,\left(d_{\kappa \iota}\right)}\left(\mathfrak{O} / \mathfrak{P}^{N}\right)$, defined in analogy to (4.10), have the property that

$$
c_{U, j,\left(d_{\kappa \iota}\right)}\left(q^{f}\right):=\left|\mathcal{C}_{U, j,\left(d_{\kappa \iota}\right)}\left(\mathbb{F}_{q^{f}}\right)\right|=\left|C_{U, j,\left(d_{\kappa \iota}\right)}\left(\mathfrak{O} / \mathfrak{P}^{N}\right)\right| .
$$

Thus we obtain

Proposition 4.4. For $I \subseteq\{1, \ldots, n-1\}$ and $\mathfrak{O} \mid \mathfrak{o}$ unramified, i.e. $e(\mathfrak{O}, \mathfrak{o})=1$, we have

$$
\begin{aligned}
Z_{\mathcal{W}(\mathfrak{O}), I}(\mathbf{s}) & =\frac{\left(1-q^{-f}\right)^{|I|}}{q^{f N n^{2}}} \sum_{\substack{\mathbf{a} \in Y\left(\mathfrak{O} / \mathfrak{P}^{N}\right) \\
h_{\mathfrak{\xi}}(\mathbf{a}) \in \mathcal{W}\left(\mathfrak{O} / \mathfrak{P}^{N}\right)}}\left(q^{f N}-q^{f(N-1)}\right)^{|U(\mathbf{a})|} q^{-f j(\mathbf{a})} \Xi_{U(\mathbf{a}),\left(d_{\kappa \iota}(\mathbf{a})\right), I}^{N}\left(q^{f}, \mathbf{s}\right) \\
& =\frac{\left(1-q^{-f}\right)^{|I|}}{q^{f N n^{2}}} \sum_{U, j,\left(d_{\kappa \iota}\right)} c_{U, j,\left(d_{\kappa \iota}\right)}\left(q^{f}\right)\left(q^{f N}-q^{f(N-1)}\right)^{|U|} q^{-f j} \Xi_{U,\left(d_{\kappa \iota}\right), I}^{N}\left(q^{f}, \mathbf{s}\right),
\end{aligned}
$$

where - denotes reduction modulo $\mathfrak{P}^{N}$.

This generalises Proposition 4.1 and its Corollary 4.2 ,

4.3.2. Totally ramified extensions. We now consider totally ramified extensions $\mathfrak{O}$ of $\mathfrak{o}$. In contrast to Section 4.3.1 where we provided, in Proposition 4.4, a uniform formula for all unramified extensions of $\mathfrak{o}$, we derive here a formula for the integral over $\mathfrak{o}$ by restriction of a formula for the integral over a fixed totally ramified extension $\mathfrak{O}$ of $\mathfrak{o}$. In fact, we do not know whether a general 'uniform' formula akin to Proposition 4.4 exists for totally ramified extensions.

Let $\mathfrak{O} \mid \mathfrak{o}$ be an extension with $f(\mathfrak{O}, \mathfrak{o})=1$ and $e=e(\mathfrak{O}, \mathfrak{o})$ arbitrary. As explained in Section 4.3 there exists $N \in \mathbb{N}$ such that we may cover $Y(\mathfrak{O})$ disjointly by cosets modulo $\mathfrak{P}^{e N}$, which are homeomorphic to $\left(\mathfrak{P}^{e N}\right)^{(b)}$, so that on each coset the integrand is 'monomial', with local numerical data $\left(U, j,\left(d_{\kappa \iota}\right)\right)$. Proposition 4.1 yields a formula for $Z_{W(\mathfrak{O}), I}(\mathbf{s})$ :

$$
Z_{\mathcal{W}(\mathfrak{O}), I}(\mathbf{s})=\frac{\left(1-q^{-1}\right)^{|I|}}{q^{e N n^{2}}} \sum_{\substack{\mathbf{a} \in Y\left(\mathfrak{O} / \mathfrak{P}^{e N}\right) \\ h_{\mathfrak{K}}(\mathbf{a}) \in \mathcal{W}\left(\mathfrak{O} / \mathfrak{P}^{e N}\right)}}\left(q^{e N}-q^{e N-1}\right)^{|U(\mathbf{a})|} q^{-j(\mathbf{a})} \Xi_{U(\mathbf{a}),\left(d_{\kappa \iota}(\mathbf{a})\right), I}^{e N}(q, \mathbf{s}) .
$$

Each summand in this sum is associated to an integral over

$$
\mathfrak{P}^{(|I|)} \times\left\{\mathbf{y} \in Y(\mathfrak{O}) \mid \mathbf{y} \equiv \mathbf{a} \bmod \left(\mathfrak{P}^{e N}\right)\right\}
$$

We consider the set $Y\left(\mathfrak{o} / \mathfrak{p}^{N}\right)$ as a subset of $Y\left(\mathfrak{O} / \mathfrak{P}^{e N}\right)$ and obtain a formula for the integral $Z_{\mathcal{W}(\mathfrak{o}), I}(\mathbf{s})$ by 'restricting' these integrals to

$$
\mathfrak{p}^{(|I|)} \times\left\{\mathbf{y} \in Y(\mathfrak{o}) \mid \mathbf{y} \equiv \mathbf{a} \bmod \left(\mathfrak{p}^{N}\right)\right\},
$$

taking into account the different normalisations of the Haar measure on $\left(\mathfrak{P}^{e N}\right)^{(b)}$ and $\left(\mathfrak{p}^{N}\right)^{(b)}$, respectively. (Recall that, for a uniformiser $\pi$ of $\mathfrak{o}$, we have $|\pi|_{\mathfrak{p}}=q^{-1}$, whereas 
$|\pi|_{\mathfrak{P}}=q^{-e}$.) We note that the sets (4.12) are non-empty only if $\mathbf{a} \in Y\left(\mathfrak{o} / \mathfrak{p}^{N}\right)$. In this case, $j(\mathbf{a}) \equiv d_{\kappa \iota}(\mathbf{a}) \equiv 0 \bmod (e)$, as all the polynomials in $F_{\kappa \iota}(\mathbf{Y})$ and the principalisation $h$ are defined over $k$. Thus

$$
Z_{\mathcal{W}(\mathfrak{o}), I}(\mathbf{s})=\frac{\left(1-q^{-1}\right)^{|I|}}{q^{N n^{2}}} \sum_{\substack{\mathbf{a} \in Y\left(\mathfrak{o} / \mathfrak{p}^{N}\right) \\ \overline{h_{\mathfrak{k}}}(\mathbf{a}) \in \mathcal{W}\left(\mathfrak{o} / \mathfrak{p}^{N}\right)}}\left(q^{N}-q^{N-1}\right)^{|U(\mathbf{a})|} q^{-j(\mathbf{a}) / e} \Xi_{U(\mathbf{a}), d_{\kappa \iota}(\mathbf{a}) / e, I}^{N}(q, \mathbf{s}) .
$$

4.4. Poles of zeta functions of polyhedral cones. The various explicit formulae for the functions $Z_{\mathcal{W}(\mathfrak{O}), I}(\mathbf{s})$ show that central to a study of the poles of these zeta integrals is an analysis of the poles of certain zeta functions of polyhedral cones.

Proposition 4.5. Let $q, f, N \in \mathbb{N}$, let $\left(\delta_{\kappa \iota}\right)_{\kappa \in\{1, \ldots, l\}, \iota \in J_{\kappa}}$ be a family of integers, $\mathbf{a}, \mathbf{b} \in$ $\mathbb{Z}^{l}, U \subseteq T, I \subseteq\{1, \ldots, n-1\}$ and let $L(\mathbf{m}, \mathbf{n})$ and $\left(L_{\kappa \iota}(\mathbf{m}, \mathbf{n})\right)$ be linear forms as defined in Section 4.1.1. Then the set of real parts of the poles of

$$
\Xi_{U,\left(\delta_{\kappa \iota}\right), I}^{N}\left(q^{f}, \mathbf{a} s+\mathbf{b}\right):=\sum_{\mathbf{m} \in \mathbb{N}_{\geq N}^{U}, \mathbf{n} \in \mathbb{N}^{I}}\left(q^{f}\right)^{L(\mathbf{m}, \mathbf{n})-\sum_{\kappa=1}^{l}\left(a_{\kappa} s+b_{\kappa}\right) \min \left\{L_{\kappa \iota}(\mathbf{m}, \mathbf{n})-\delta_{\kappa \iota} \mid \iota \in J_{\kappa}\right\}}
$$

is independent of $q, f, N$ and $\left(\delta_{\kappa \iota}\right)$.

Proof. We first observe that

$$
\Xi_{U,\left(\delta_{\kappa \iota}\right), I}^{N}\left(q^{f}, \mathbf{a} s+\mathbf{b}\right)=q^{\sum_{u} \nu_{u}(1-N)} \Xi_{U,\left(\delta_{\kappa \iota}+\sum_{u} N_{u \kappa \iota}(N-1)\right), I}^{1}\left(q^{f}, \mathbf{a} s+\mathbf{b}\right),
$$

so we may assume $N=1$, and drop the superscript.

For $\left(\delta_{\kappa \iota}\right)=(0)$, recall the decomposition of $\mathbb{R}_{>0}^{|U|+|I|}$ by the family $\mathfrak{C}$ of rational polyhedral cones described in Section 4.1.1. It implies that the denominators of the generating functions $\Xi_{U, I}\left(q^{f}, \mathbf{a} s+\mathbf{b}\right)$ divide products of the form $\prod_{i \in \mathfrak{I}(U)}\left(1-\left(q^{f}\right)^{A_{i}-B_{i} s}\right)$, for some finite index sets $\mathfrak{I}(U)$ and nonnegative integers $A_{i}, B_{i}$, which only depend on $L(\mathbf{m}, \mathbf{n})$, $\left(L_{\kappa \iota}(\mathbf{m}, \mathbf{n})\right.$ ), a and $\mathbf{b}$ (but not, for instance, on $q^{f}$ ). Note that the fact that the union of the closed cones $\mathcal{C}_{g}$ in Section 4.1.1 is not disjoint is insubstantial: by construction, if two such cones do not coincide then they overlap only on components of their boundaries, and the summation over these boundary components does not introduce new denominators. The latter follows from the fact that, for each of the polyhedral cones $\mathfrak{C}_{g}$, the denominator of the generating function associated to $\mathfrak{C}_{g}$ may be described in terms of the primitive vectors on the extremal rays of $\mathcal{C}_{g}$; cf. [49, Proposition 4.6.10, Theorem 4.6.11]. We set $P:=\bigcup_{U \subseteq T}\left\{A_{i} / B_{i} \mid i \in \mathfrak{I}(U)\right\}$.

In the case of general $\left(\delta_{\kappa \iota}\right)$ we consider the affine translates

$$
L_{\kappa \iota}^{\prime}(\mathbf{m}, \mathbf{n}):=L_{\kappa \iota}(\mathbf{m}, \mathbf{n})-\delta_{\kappa \iota}=\sum_{i \in I} e_{i \kappa \iota} n_{i}+\sum_{u \in U} N_{u \kappa \iota} m_{u}-\delta_{\kappa \iota}
$$

of the linear forms $L_{\kappa \iota}(\mathbf{m}, \mathbf{n})$ defined in Section 4.1. For $g=\left(g_{\kappa}\right) \in \prod_{\kappa} J_{\kappa}$, let $\mathcal{C}_{g}^{\prime}$ be the cone in $\mathbb{R}_{>0}^{|U|+|I|}$ defined by the condition

$$
\forall \kappa \in\{1, \ldots, l\} \forall \iota \in J_{\kappa}: L_{\kappa g_{\kappa}}^{\prime} \leq L_{\kappa \iota}^{\prime} .
$$

Each cone $\mathfrak{C}_{g}^{\prime}$ in the family $\mathfrak{C}^{\prime}:=\left(\mathfrak{C}_{g}^{\prime}\right)_{g \in \prod_{\kappa} J_{\kappa}}$ is an affine translate of a cone in $\mathfrak{C}$. In continuation of our discussion in Section 4.1.1, the restriction of the summation to each $\mathrm{C}_{g}^{\prime} \cap \mathbb{N}^{|U|+|I|}$ can be interpreted in terms of the Hilbert series associated to the solutions of an inhomogeneous system $A_{g} \mathbf{x}=\boldsymbol{\beta}$, whilst the summation over the corresponding cone $\mathcal{C}_{g} \cap \mathbb{N}^{|U|+|I|}$ is interpretable in terms of the Hilbert series associated to the solutions of $A_{g} \mathbf{x}=\mathbf{0}$. However, it is known (cf. [48, I.3]) that the Hilbert series associated to the inhomogeneous system $A_{g} \mathbf{x}=\boldsymbol{\beta}$ is either 0 or else has the same denominator as the 
Hilbert series associated to $A_{g} \mathbf{x}=0$. The former clearly does not occur in the current context.

Remark 4.6. A formula for Igusa local zeta functions for good reduction which is uniform under field extensions is given in [13]. Igusa-type zeta integrals at places with bad reduction have been studied in [15]. We note that [15, Proposition 3.3] seems to be incorrect; the integrals $\int_{\mathbb{Z}_{p}}|p|_{p}^{-s} d \mu(x)$ and $\int_{\left\{(x, y) \in \mathbb{Z}_{p}^{2}|x| p y\right\}}|x y|_{p}^{-s} d \mu(x, y)$ provide counterexamples. These notwithstanding, [15, Corollary 3.4] remains valid, e.g. by arguments akin to the ones given in our proof of Theorem B,

4.5. Proofs of Theorem $\mathbf{A}$ and $\mathbf{B}$. Theorem $\mathrm{A}$ holds, with $S$ and $\lambda_{1}, \ldots, \lambda_{r}$ defined in Sections 4.1 and 4.2, because of (4.8) in conjunction with (4.7).

The first claim of Theorem $\mathrm{B}$ follows, with $P$ defined as in the proof of Proposition 4.5, from the formulae we gave for the integrals $Z_{\mathcal{W}(\mathfrak{O}), I}(\mathbf{s})$ in Sections 4.1 to 4.3 , together with Proposition 4.5.

For the second claim of Theorem $\mathrm{B}$ we note that it suffices to consider places $v \notin S$. The claim follows from the fact that, for each $U \subseteq T$, the numbers $c_{U}\left(q^{f}\right)$ are non-zero for a set of places $v$ of $k$ of positive density. This in turn is a consequence of the fact that the varieties $E_{U}$ are smooth, quasi-projective varieties defined over $k$ which are irreducible over $k$. This implies that, for every $U \subseteq T$, the numbers $b_{U}(q)$ are non-zero for a set of places of positive density. In the special case $k=\mathbb{Q}$ this follows essentially from the Lang-Weil estimate; see e.g. [15, Lemma 4.7], which is easily generalised to arbitrary number fields.

The last claim of Theorem $\mathrm{B}$ follows (i) in the case that $v \notin S$ from the monotonicity of the numbers $c_{U}\left(q^{f}\right)$ under residue field extensions and (ii) in the case that $v \in S$ from the monotonicity of the numbers $c_{U, j,\left(d_{\kappa \iota}\right)}\left(q^{f}\right)$ in the case of unramified extensions, and from the discussion in Section 4.3 .2 and Proposition 4.5 in the totally ramified case.

\section{Semisimple Groups}

In this section we establish a link between the representation zeta functions of 'semisimple' $p$-adic analytic pro- $p$ groups and stratifications of the associated semisimple Lie algebras by algebraic varieties defined in terms of centraliser dimension.

Let $\mathcal{O}$ be the ring of integers of a number field $k$, and $\Lambda$ an $\mathcal{O}$-Lie lattice such that $\mathcal{L}:=k \otimes_{\mathcal{O}} \Lambda$ is a finite dimensional, perfect $k$-Lie algebra. Put $d:=\operatorname{dim}_{k}(\mathcal{L})$. We choose an $\mathcal{O}$-basis $\mathbf{b}=\left(b_{1}, \ldots, b_{d}\right)$ of $\Lambda$, and we define the commutator matrix $\mathcal{R}(\mathbf{Y})$ of $\Lambda$ with respect to the basis $\mathbf{b}$ as in (3.1).

For any non-archimedean place $v$ of $k$, the completion $\mathfrak{o}=\mathcal{O}_{v}$ is a compact discrete valuation ring of characteristic 0 , with maximal ideal $\mathfrak{p}$ and residue field $\mathfrak{o} / \mathfrak{p}$ of cardinality $q$ and characteristic $p$, say. The $\mathfrak{o}$-Lie lattice $\mathfrak{g}:=\mathfrak{o} \otimes_{\mathcal{O}} \Lambda$ gives rise to a family of FAb $p$-adic analytic pro- $p$ groups $\mathrm{G}^{m}(\mathfrak{o}), m \in \mathbb{N}_{0}$ permissible for $\mathfrak{g}$. Equation (3.4) expresses the relevant Poincaré series $\mathcal{P}_{\mathcal{R}, \mathfrak{o}}(s)$ in terms of the generalised Igusa local zeta function $z_{\mathfrak{o}}(r, t)$. If $\mathcal{L}$ is semisimple, then the parameter $\rho$ defined in (3.6) has a natural interpretation in terms of the absolute root system and the absolute rank of the Lie algebra $\mathcal{L}$. These are the root system $\Phi\left(\mathcal{L}_{\mathbb{C}}\right)$ and the rank $\mathrm{r}\left(\mathcal{L}_{\mathbb{C}}\right)$ of the complex semisimple Lie algebra $\mathcal{L}_{\mathbb{C}}:=\mathbb{C} \otimes_{k} \mathcal{L}$. Indeed, we have $2 \rho=d-\mathrm{r}\left(\mathcal{L}_{\mathbb{C}}\right)=\left|\Phi\left(\mathcal{L}_{\mathbb{C}}\right)\right|$.

Looking in more detail at the integrand in (3.5), we observe that the following stratification of $d$-dimensional affine space $\mathbb{A}^{d}$ plays a significant role:

$$
\mathbb{A}^{d}=\mathcal{U}_{0} \supseteq \mathcal{U}_{1} \supseteq \ldots \supseteq \mathcal{U}_{\rho}=\{0\},
$$


where for $i \in\{0, \ldots, \rho\}$ the subvariety $\mathcal{U}_{i}$ is defined by the set of polynomials $F_{\rho+1-i}(\mathbf{Y})$, over $\mathcal{O}$. We note that for each $i$ in this range

$$
\begin{aligned}
\mathcal{U}_{i}(k) & =\left\{\underline{x} \in k^{d} \mid \forall f \in F_{\rho+1-i}(\mathbf{Y}): f(\underline{x})=0\right\} \\
& =\left\{\underline{x} \in k^{d} \mid \operatorname{rk}_{k}(\mathcal{R}(\underline{x})) \leq d-\mathrm{r}\left(\mathcal{L}_{\mathbb{C}}\right)-2 i\right\} .
\end{aligned}
$$

We now make the assumption that the Lie algebra $\mathcal{L}$ is semisimple. It is remarkable that in this important case the stratification above can be given a natural algebraic interpretation, as we shall now explain. (Our description makes use of properties of the Killing form, namely that it is non-degenerate and invariant; more generally, it applies to any Lie lattice such that $\mathcal{L}$ admits some non-degenerate invariant symmetric bilinear form.)

By a choice of co-ordinates, we identify the $k$-vector spaces $\mathcal{L}$ and and its dual $\mathcal{L}^{\vee}$ with the $k$-points of $d$-dimensional affine space $\mathbb{A}^{d}$. Indeed, the $\mathcal{O}$-basis $\mathbf{b}$ of $\mathcal{L}$ and the dual o-basis $\mathbf{b}^{\vee}$ of $\mathcal{L}^{\vee}$ induce co-ordinate maps

$$
\begin{aligned}
& \mathcal{L} \stackrel{\simeq}{\longrightarrow} k^{d}, \quad x=\sum_{i=1}^{d} x_{i} b_{i} \mapsto \underline{x}=\left(x_{1}, \ldots, x_{d}\right), \\
& \mathcal{L}^{\vee} \stackrel{\simeq}{\longrightarrow} k^{d}, \quad x=\sum_{i=1}^{d} x_{i} b_{i}^{\vee} \mapsto \underline{x}=\left(x_{1}, \ldots, x_{d}\right),
\end{aligned}
$$

defined over $\mathcal{O}$. The image $\omega_{\underline{x}}=\sum_{i=1}^{d} x_{i} b_{i}^{\vee}$ of $\underline{x}$ under the inverse of the second isomorphism is uniquely defined by the requirements

$$
\omega_{\underline{x}}\left(b_{i}\right)=x_{i} \quad \text { for } i \in\{1, \ldots, d\} .
$$

This allows us to interpret the stratification of $\mathbb{A}^{d}$ in terms of $\mathcal{U}_{i}, i \in\{0, \ldots, \rho\}$, by a stratification $\mathcal{W}_{i}, i \in\{0, \ldots, \rho\}$, of $\mathbb{A}^{d}$ which is also defined over $\mathcal{O}$ and satisfies

$$
\mathcal{W}_{i}(k)=\left\{\omega_{\underline{x}} \in \mathcal{L}^{\vee} \mid \operatorname{rk}_{k}(\mathcal{R}(\underline{x})) \leq d-\operatorname{r}\left(\mathcal{L}_{\mathbb{C}}\right)-2 i\right\} .
$$

As $\mathcal{L}$ is semisimple, there is also a canonical vector space isomorphism from $\mathcal{L}$ to its dual $\mathcal{L}^{\vee}$ via the Killing form. It is well-known that the Killing form

$$
\kappa: \mathcal{L} \times \mathcal{L} \longrightarrow k, \quad \kappa(x, y):=\operatorname{Tr}(\operatorname{ad}(x) \operatorname{ad}(y))
$$

of the semisimple Lie algebra $\mathcal{L}$ is non-degenerate and thus induces a natural isomorphism of vector spaces

$$
\iota: \mathcal{L} \longrightarrow \mathcal{L}^{\vee}, \quad x \mapsto \kappa(x, \cdot) .
$$

We note that, while $\iota$ is defined over $\mathcal{O}$, its inverse is typically not defined over $\mathcal{O}$. This issue will be discussed more closely below.

In any case, we claim that $\mathcal{W}_{i}, i \in\{0, \ldots, \rho\}$, is the image under $\iota$ of the stratification

$$
\mathbb{A}^{d}=\mathcal{V}_{0} \supseteq \mathcal{V}_{1} \supseteq \ldots \supseteq \mathcal{V}_{\rho}=\{0\},
$$

where

$$
\mathcal{V}_{i}(k)=\left\{x \in \mathcal{L} \mid \operatorname{dim}_{k} \mathrm{C}_{\mathcal{L}}(x) \geq \mathrm{r}\left(\mathcal{L}_{\mathbb{C}}\right)+2 i\right\} .
$$

The non-degeneracy and invariance of the Killing form $\kappa$ show that for $x \in \mathcal{L}$ the radical $\operatorname{Rad}(\kappa(x, \cdot))$ is equal to the centraliser $\mathrm{C}_{\mathcal{L}}(x)$. Indeed, we have

$$
\begin{aligned}
\operatorname{Rad}(\kappa(x, \cdot)) & =\{y \in \mathcal{L} \mid \forall z \in \mathcal{L}: \kappa(x,[y, z])=0\} \\
& =\{y \in \mathcal{L} \mid \forall z \in \mathcal{L}: \kappa([x, y], z)=0\} \\
& =\{y \in \mathcal{L} \mid[x, y]=0\} \\
& =\mathrm{C}_{\mathcal{L}}(x) .
\end{aligned}
$$


This implies that, for $i \in\{0, \ldots, \rho\}$,

$$
\begin{aligned}
\iota \mathcal{V}_{i}(k) & =\left\{\kappa(x, \cdot) \in \mathcal{L}^{\vee} \mid \operatorname{dim}_{k} \operatorname{Rad}(\kappa(x, \cdot)) \geq \mathrm{r}\left(\mathcal{L}_{\mathbb{C}}\right)+2 i\right\} \\
& =\left\{\omega_{\underline{x}} \in \mathcal{L}^{\vee} \mid \operatorname{dim}_{k} \operatorname{Rad}\left(\omega_{\underline{x}}\right) \geq \mathrm{r}\left(\mathcal{L}_{\mathbb{C}}\right)+2 i\right\} .
\end{aligned}
$$

Now let $\omega_{\underline{x}} \in \mathcal{L}^{\vee}$ with co-ordinates $\underline{x}$. Note that for $y, z \in \mathcal{L}$,

$$
\omega_{\underline{x}}([y, z])=\underline{y} \cdot \mathcal{R}(\underline{x}) \cdot \underline{z}^{\mathrm{t}} .
$$

Therefore the radical $\operatorname{Rad}\left(\omega_{\underline{x}}\right)=\left\{y \in \mathcal{L} \mid \forall z \in \mathcal{L}: \omega_{\underline{x}}([y, z])=0\right\}$ maps under the co-ordinate isomorphism $\mathcal{L} \simeq k^{d}, y \mapsto \underline{y}$ onto ker $\mathcal{R}(\underline{x})$. From the fact that $d=\operatorname{dim}_{k} \operatorname{Rad}\left(\omega_{\underline{x}}\right)+\operatorname{rk}_{k}(\mathcal{R}(\underline{x}))$ we deduce that, for $i \in\{0, \ldots, \rho\}$,

$$
\mathcal{W}_{i}(k)=\left\{\omega_{\underline{x}} \in \mathcal{L}^{\vee} \mid \operatorname{dim}_{k} \operatorname{Rad}\left(\omega_{\underline{x}}\right) \geq \operatorname{r}\left(\mathcal{L}_{\mathbb{C}}\right)+2 i\right\} .
$$

From (5.1) and (5.2) we deduce by extension of scalars that the stratification $\mathcal{V}_{i}$, $i \in\{0, \ldots, \rho\}$, is mapped to $\mathcal{W}_{i}, i \in\{0, \ldots, \rho\}$, under the linear isomorphism $\iota$.

In practice, the translation between the varieties needs to be carried out locally, over the ring $\mathfrak{o}=\mathcal{O}_{v}$. Instead of the Killing form we use - in the case of a simple Lie algebra of Chevalley type - the normalised Killing form $\kappa_{0}$ which is related to the ordinary Killing form $\kappa$ by the equation $2 h^{\vee} \kappa_{0}=\kappa$ where $h^{\vee}$ is the dual Coxeter number. For instance, for $\mathfrak{s l}_{n}$ the dual Coxeter number is $h^{\vee}=n$.

A good understanding of the representation zeta functions of 'semisimple' $p$-adic analytic pro- $p$ groups would depend on detailed knowledge of algebro-geometric and arithmetic properties of the projective subvarieties of $\mathbb{P}(\mathcal{L}) \cong \mathbb{P}^{d-1}(\mathbb{C})$ defined by the affine varieties $\mathcal{V}_{i}, i \in\{0, \ldots, \rho\}$. Indeed, the discussion in Section 4 shows that explicit formulae for the relevant zeta functions may be obtained from a principalisation of ideals defining the terms of this filtration. The theory of sheets may be relevant in this context; cf. [6]. In the first place we would like to understand the geometry of this stratification over $\mathbb{C}$ (or any algebraically closed field above $k$ ), and in particular its singular loci. In a second step one would need to describe the varieties over completions $\mathfrak{o}=\mathcal{O}_{v}$ of $\mathfrak{O}$. We put forward the following concrete question.

Question 5.1. Let $\mathcal{L}_{\mathbb{C}}$ be a complex semisimple Lie algebra of dimension $d$, representing $\mathbb{A}^{d}(\mathbb{C})$. Consider the stratification $\mathbb{A}^{d}=\mathcal{V}_{0} \supseteq \mathcal{V}_{1} \supseteq \ldots \supseteq \mathcal{V}_{\rho}=\{0\}$, where $2 \rho=$ $d-\mathrm{r}\left(\mathcal{L}_{\mathbb{C}}\right)=\left|\Phi\left(\mathcal{L}_{\mathbb{C}}\right)\right|$ and

$$
\mathcal{V}_{i}(\mathbb{C})=\left\{x \in \mathcal{L}_{\mathbb{C}} \mid \operatorname{dim}_{\mathbb{C}} \mathrm{C}_{\mathcal{L}_{\mathbb{C}}}(x) \geq \mathrm{r}\left(\mathcal{L}_{\mathbb{C}}\right)+2 i\right\} \quad \text { for } i \in\{0, \ldots, \rho\} .
$$

Is it true that the singular locus of any non-smooth term $\mathcal{V}_{i}$ in this stratification is given by the first successor term $\mathcal{V}_{j}, j>i$, which is properly contained in $\mathcal{V}_{i}$ ?

Already for $\mathcal{L}_{\mathbb{C}}=\mathfrak{s l}_{4}(\mathbb{C})$, the singularities of the varieties $\mathcal{V}_{i}$ are challenging. The case of Lie algebras of type $A_{2}$, however, is understood.

Example 5.2. Let $\mathcal{L}_{\mathbb{C}}=\mathfrak{s l}_{3}(\mathbb{C})$. Hence $d=8$, and it is not hard to verify that $\rho=3$. Consider the map

$$
\beta_{\mathbb{C}}: \mathfrak{g l}_{3}(\mathbb{C}) \rightarrow \mathfrak{s l}_{3}(\mathbb{C}), \quad X \mapsto X-\frac{\operatorname{Tr}(X)}{3} \operatorname{Id}_{3},
$$

and set $\mathcal{D}_{1}(\mathbb{C}):=\left\{X \in \mathfrak{g l}_{3}(\mathbb{C}) \mid \operatorname{rk}_{\mathbb{C}}(X) \leq 1\right\}$. Regarding the filtration $\mathbb{A}^{8}=\mathcal{V}_{0} \supseteq$ $\mathcal{V}_{1} \supseteq \mathcal{V}_{2} \supseteq \mathcal{V}_{3}=\{0\}$ it is known that $\mathcal{V}_{2}(\mathbb{C})=\{0\}$ and that $\mathcal{V}_{1}(\mathbb{C})=\beta_{\mathbb{C}}\left(\mathcal{D}_{1}(\mathbb{C})\right)$; cf. [8, Example 9.6]. The variety $\mathcal{V}_{1}$ is a 5 -dimensional subvariety of $\mathbb{A}^{8}$, defined over $\mathbb{Z}$, which is smooth away from the origin. The projective variety $\mathbb{P}\left(\mathcal{V}_{1} \backslash\{0\}\right)$ is isomorphic to $\mathbb{P}^{2} \times \mathbb{P}^{2}$. 
The straightforward geometric setup of Example 5.2 holds the key to our analysis of Lie algebras of type $A_{2}$ in Section [6, enabling us to compute explicit formulae for the corresponding zeta functions.

\section{Part 2. Applications}

\section{Formulae for PRincipal CONGRUEnce SubGroups of $\mathrm{SL}_{3}(\mathfrak{o})$ and $\operatorname{SU}_{3}(\mathfrak{O}, \mathfrak{o})$}

In this section we use the methods developed in Part 1 to prove Theorem E. We first fix some notation, mainly for the unitary case. Let $\mathfrak{o}$ denote a compact discrete valuation ring of characteristic 0 with field of fractions $\mathfrak{k}$, and let $\mathfrak{K}$ be an unramified quadratic extension of $\mathfrak{k}$, with ring of integers $\mathfrak{O}$. We write $\mathfrak{p}$ and $\mathfrak{P}$ for the maximal ideals of $\mathfrak{o}$ and $\mathfrak{O}$; the residue field characteristic and cardinality of $\mathfrak{o}$ are denoted by $p$ and $q$. We write $\mathbb{F}_{q}$ for the residue field $\mathfrak{o} / \mathfrak{p}$.

Let $\sigma: \mathfrak{K} \rightarrow \mathfrak{K}$ denote the non-trivial Galois automorphism of $\mathfrak{K} \mid \mathfrak{k}$. For $n \in \mathbb{N}$, consider on $V=\mathfrak{K}^{n}$ the standard non-degenerate Hermitian sesquilinear form $V \times V \rightarrow \mathfrak{K}$, $(\mathbf{v}, \mathbf{w}) \mapsto \sum_{i=1}^{n} v_{i}^{\sigma} w_{i}$. The structure matrix of this form with respect to the standard basis is simply the identity matrix. The standard involution ${ }^{\circ}$ on the matrix algebra $\operatorname{Mat}_{n}(\mathfrak{K})$ is given by $\mathbf{x}^{\circ}:=\left(\mathbf{x}^{\sigma}\right)^{\mathrm{t}}$, i.e. conjugate transpose. The standard unitary group $\mathrm{GU}_{n}(\mathfrak{K}, \mathfrak{k})$ and its subgroups, e.g. the standard special unitary group $\mathrm{SU}_{n}(\mathfrak{K}, \mathfrak{k})$, can be realised as subgroups of $\mathrm{GL}_{n}(\mathfrak{K})$ as follows:

$$
\mathrm{GU}_{n}(\mathfrak{K}, \mathfrak{k})=\left\{g \in \mathrm{GL}_{n}(\mathfrak{K}) \mid g^{\circ} g=1\right\} .
$$

Similarly, the standard unitary Lie algebra $\mathfrak{g u}_{n}(\mathfrak{K}, \mathfrak{k})$ and its subalgebras, e.g. the standard special unitary Lie algebra $\mathfrak{s u}_{n}(\mathfrak{K}, \mathfrak{k})$, can be realised as subalgebras of the $\mathfrak{k}$-Lie algebra $\mathfrak{g l}_{n}(\mathfrak{K})$ :

$$
\mathfrak{g} \mathfrak{u}_{n}(\mathfrak{K}, \mathfrak{k})=\left\{\mathbf{x} \in \mathfrak{g u _ { n }}(\mathfrak{K}) \mid \mathbf{x}^{\circ}+\mathbf{x}=0\right\} .
$$

We define the compact $p$-adic analytic group

$$
\mathrm{SU}_{n}(\mathfrak{O}, \mathfrak{o}):=\mathrm{SU}_{n}(\mathfrak{K}, \mathfrak{k}) \cap \mathrm{GL}_{n}(\mathfrak{O})
$$

and the $\mathfrak{o}$-Lie lattice

$$
\mathfrak{s u}_{n}(\mathfrak{O}, \mathfrak{o}):=\mathfrak{s u}_{n}(\mathfrak{K}, \mathfrak{k}) \cap \mathfrak{g l}_{n}(\mathfrak{O}) .
$$

Throughout the remainder of this section, we will assume that the residue field characteristic of $\mathfrak{o}$ is not equal to 3, i.e. that $p \neq 3$. Under this restriction, we compute explicit formulae for the representation zeta functions of the principal congruence subgroups $\mathrm{SL}_{3}^{m}(\mathfrak{o})$, where $m$ is permissible for $\mathfrak{s l}_{3}(\mathfrak{o})$, in Section 6.1 and of the principal congruence subgroups $\mathrm{SU}_{3}^{m}(\mathfrak{O}, \mathfrak{o})$, where $m$ is permissible for $\mathfrak{s u}_{3}^{m}(\mathfrak{O}, \mathfrak{o})$, in Section 6.2 . Calculations of zeta functions associated to principle congruence subgroups of $\mathrm{SL}_{3}(\mathfrak{o})$, where $\mathfrak{o}$ is an unramified extension of $\mathbb{Z}_{3}$, can be found in [4].

6.1. Principal congruence subgroups of $\mathrm{SL}_{3}(\mathfrak{o})$. In this section we prove the first part of Theorem E we compute explicit formulae for the representation zeta functions of the principal congruence subgroups $\operatorname{SL}_{3}^{m}(\mathfrak{o})$, where $m$ is permissible for $\mathfrak{s l}_{3}(\mathfrak{o})$. For this we compute the integral (3.5) over $\mathfrak{p} \times W(\mathfrak{o})$, where $W(\mathfrak{o})=\left(\mathfrak{o}^{8}\right)^{*} \cong \operatorname{Hom}_{\mathfrak{o}}\left(\mathfrak{s l}_{3}(\mathfrak{o}), \mathfrak{o}\right)^{*}$. The explicit computation of this integral is feasible because of two facts. Firstly, only the family $F_{3}(\mathbf{Y})$ gives a non-trivial contribution in the integrand and, secondly, the projective subvariety of $\mathbb{P}^{7}$ determined by the ideal generated by $F_{3}(\mathbf{Y})$ is smooth. To 
be concrete, one may form the commutator matrix $\mathcal{R}(\mathbf{Y})$ and the sets of polynomials $F_{j}(\mathbf{Y}), j \in\{0, \ldots, \rho\}$, with respect to the $\mathfrak{o}$-basis $\mathbf{b}$ for $\mathfrak{s l}_{3}(\mathfrak{o})$ comprising the elements

$$
\begin{aligned}
& \mathbf{h}_{12}=\left(\begin{array}{ccc}
1 & & \\
& -1 & \\
& & 0
\end{array}\right), \quad \mathbf{h}_{23}=\left(\begin{array}{ccc}
0 & & \\
& 1 & \\
& & -1
\end{array}\right),
\end{aligned}
$$

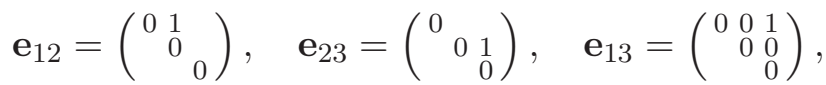

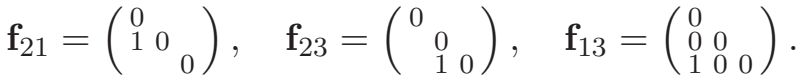

In fact, the argument we carry out below is co-ordinate free.

Recall the definition (3.6) of the parameter $\rho$. It takes a short computation to verify that, in the current context, $\rho=3$ and that $\max \left\{|f(\mathbf{y})|_{\mathfrak{p}} \mid f \in F_{2}(\mathbf{Y})\right\}=1$ for all $\mathbf{y} \in W(\mathfrak{o})=\left(\mathfrak{o}^{8}\right)^{*} ;$ cf. Example 5.2. It therefore suffices to compute the integral

$$
z_{\mathfrak{o}}(r, t)=\int_{(x, \mathbf{y}) \in \mathfrak{p} \times W(\mathfrak{o})}|x|_{\mathfrak{p}}^{t}\left\|F_{3}(\mathbf{y}) \cup\left\{x^{2}\right\}\right\|_{\mathfrak{p}}^{r} d \mu(x, \mathbf{y}) .
$$

In accordance with Section 5 , the affine variety $\mathcal{W}_{1}$ defined by $F_{3}(\mathbf{Y})$ corresponds to the subvariety $\mathcal{V}_{1}$ of $\mathfrak{s l}_{3}$ whose $\mathfrak{k}$-points coincide with the irregular elements in the 8dimensional Lie algebra $\mathfrak{s l}_{3}(\mathfrak{k})$. The translation is carried out by means of the normalised Killing form $\kappa_{0}(\cdot, \cdot)=6^{-1} \kappa(\cdot, \cdot)$ which can be presented, with respect to the basis $\mathbf{b}$, by the non-degenerate symmetric matrix

$$
\left[\kappa_{0}(\cdot, \cdot)\right]_{\mathbf{b}}=\left(\begin{array}{cccccc}
2 & -1 & & & & \\
-1 & 2 & & & & \\
& & & & & \\
& & & & & 1 \\
& & 1 & & & \\
& & & 1 & &
\end{array}\right) .
$$

Since $p \neq 3$, we conclude that $\kappa_{0}$ induces an isomorphism of $\mathfrak{o}$-modules between $\mathfrak{s l}_{3}(\mathfrak{o})^{*}$ and $\operatorname{Hom}_{\mathfrak{o}}\left(\mathfrak{s l}_{3}(\mathfrak{o}), \mathfrak{o}\right)^{*}$.

It is known (cf. Example 5.2) that the irregular locus $\mathcal{V}_{1}$ of $\mathfrak{s l}_{3}$ may be realised as the image of the variety $\mathcal{D}_{1}$ of matrices of rank at most 1 under the projection

$$
\beta: \mathfrak{g l}_{3} \rightarrow \mathfrak{s l}_{3}, \quad \mathbf{x} \mapsto \mathbf{x}-\frac{\operatorname{Tr}(\mathbf{x})}{3} \operatorname{Id}_{3} .
$$

In the language of sheets (cf. [6]), the image $\beta\left(\mathcal{D}_{1}\right)$ is the union of the sheets associated to the partitions $(1,1,1)$ and $(2,1)$ of 3 , that is the null-sheet and the unique subregular sheet. One verifies that $\left.\beta\right|_{\mathcal{D}_{1}}$ is injective, and $\beta$ induces a morphism $\beta_{\mathfrak{o}}: \mathfrak{g l}_{3}(\mathfrak{o}) \rightarrow \mathfrak{s l}_{3}(\mathfrak{o})$ whose restriction to $\mathcal{D}_{1}(\mathfrak{o})$ has good reduction modulo $\mathfrak{p}$. Moreover, one easily checks that $\beta_{\mathfrak{p}}$ maps $\mathcal{D}_{1}(\mathfrak{o})$ onto $\mathcal{V}_{1}(\mathfrak{o})$. Thus $\mathcal{V}_{1}(\mathfrak{o})=\beta_{\mathfrak{p}}\left(\mathcal{D}_{1}(\mathfrak{o})\right)$ is an affine cone which is smooth away from the origin. The number of $\mathbb{F}_{q}$-rational points of $\overline{\mathcal{V}_{1}}$, viz. the reduction of $\mathcal{V}_{1}$ modulo $\mathfrak{p}$, is

$$
\left|\overline{\mathcal{V}_{1}}\left(\mathbb{F}_{q}\right)\right|=\left|\overline{\mathcal{D}_{1}}\left(\mathbb{F}_{q}\right)\right|=\left|\left(\mathbb{P}^{2} \times \mathbb{P}^{2}\right)\left(\mathbb{F}_{q}\right)\right|(q-1)+1=\left(q^{2}+q+1\right)^{2}(q-1)+1 .
$$

To evaluate the integral (6.2), we proceed in the spirit of the proof of [11, Theorem 3.1]. The factor $W(\mathfrak{o})$ in the domain of integration may be written as a disjoint union of domains of fixed residue modulo $\mathfrak{p}$ : for each $\mathbf{a} \in\left(\mathbb{F}_{q}^{8}\right)^{*}$, we set $W_{\mathbf{a}}(\mathfrak{o}):=\{\mathbf{y} \in W(\mathfrak{o}) \mid$ $\left.\mathbf{y} \equiv_{\mathfrak{p}} \mathbf{a}\right\}$ and

$$
\begin{aligned}
z_{\mathfrak{o}, \mathbf{a}}(r, t) & :=\int_{(x, \mathbf{y}) \in \mathfrak{p} \times W_{\mathbf{a}}(\mathfrak{o})}|x|_{\mathfrak{p}}^{t}\left\|F_{3}(\mathbf{y}) \cup\left\{x^{2}\right\}\right\|_{\mathfrak{p}}^{r} d \mu(x, \mathbf{y}) \\
& =\int_{(x, \widetilde{\mathbf{y}}) \in \mathfrak{p}^{(9)}}|x|_{\mathfrak{p}}^{t}\left\|F_{3}(\mathbf{a}+\widetilde{\mathbf{y}}) \cup\left\{x^{2}\right\}\right\|_{\mathfrak{p}}^{r} d \mu(x, \widetilde{\mathbf{y}})
\end{aligned}
$$


so that $W(\mathfrak{o})=\dot{\bigcup}_{\mathbf{a} \in\left(\mathbb{F}_{q}^{8}\right)^{*}} W_{\mathbf{a}}(\mathfrak{o})$ implies

$$
z_{\mathfrak{o}}(r, t)=\sum_{\mathbf{a} \in\left(\mathbb{F}_{q}^{8}\right)^{*}} z_{\mathfrak{o}, \mathbf{a}}(r, t)
$$

We call a point $\mathbf{a} \in\left(\mathbb{F}_{q}^{8}\right)^{*}$ and any $\mathbf{y} \in W_{\mathbf{a}}(\mathfrak{o})$ regular if $\mathbf{a}$ is not an $\mathbb{F}_{q^{-} \text {-rational }}$ point of $\overline{\mathcal{V}_{1}}$. A functional $w \in \operatorname{Hom}_{\mathfrak{o}}\left(\mathfrak{s l}_{3}(\mathfrak{o}), \mathfrak{o}\right)^{*}$ and the representations associated to the Kirillov orbits of the images of $w$ in $\operatorname{Hom}_{\mathfrak{o}}\left(\mathfrak{s l}_{3}(\mathfrak{o}), \mathfrak{o} / \mathfrak{p}^{n}\right)^{*}, n \in \mathbb{N}$, are said to be regular if the co-ordinate vector $\mathbf{y} \in W(\mathfrak{o})$ corresponding to $w$ is regular. The computation of $\boldsymbol{z}_{\mathfrak{0}, \mathbf{a}}(r, t)$ is particularly straightforward if $\mathbf{a} \in\left(\mathbb{F}_{q}^{8}\right)^{*}$ is regular: in this case $z_{\mathfrak{o}, \mathbf{a}}(r, t)=z_{\mathfrak{o}}^{[0]}(r, t)$, where

$$
z_{\mathfrak{o}}^{[0]}(r, t):=\int_{(x, \widetilde{\mathbf{y}}) \in \mathfrak{p}^{(9)}}|x|_{\mathfrak{p}}^{t} d \mu(x, \widetilde{\mathbf{y}})=\frac{q^{-9-t}\left(1-q^{-1}\right)}{1-q^{-1-t}} .
$$

Next we consider the more complex situation where $\mathbf{a} \in\left(\mathbb{F}_{q}^{8}\right)^{*}$ is an $\mathbb{F}_{q}$-rational point of $\overline{V_{1}}$. In this case the point $\mathbf{a}$, any co-ordinate vector $\mathbf{y} \in W_{\mathbf{a}}(\mathfrak{o})$, the functionals $w \in \operatorname{Hom}_{\mathfrak{o}}\left(\mathfrak{s l}_{3}(\mathfrak{o}), \mathfrak{o}\right)^{*}$ represented by such $\mathbf{y}$ and the representations associated to the Kirillov orbits of the images of such $w$ in $\operatorname{Hom}_{\mathfrak{o}}\left(\mathfrak{s l}_{3}(\mathfrak{o}), \mathfrak{o} / \mathfrak{p}^{n}\right)^{*}, n \in \mathbb{N}$, are said to be irregular.

We observe that by a suitable transformation of the co-ordinate functions $\mathbf{y}$ we may simplify the integral defining $z_{\mathfrak{o}, \mathbf{a}}(r, t)$ significantly. Indeed, recall that, by Remark 3.6, we may replace $F_{3}(\mathbf{Y})$ by the set of principal $6 \times 6$-minors of the commutator matrix $\mathcal{R}(\mathbf{Y})$. These minors are squares. Near a smooth point (in the case under consideration any point away from the origin; cf. Example 5.2) we may thus replace the set of functions $F_{3}(\mathbf{Y})$ defining the 5 -dimensional variety $\mathcal{V}_{1}$ by the simpler set $\left\{y_{1}^{2}, y_{2}^{2}, y_{3}^{2}\right\}$ consisting of the squares of the first three co-ordinate functions, say. This transformation yields that $z_{\mathfrak{o}, \mathbf{a}}(r, t)=z_{\mathfrak{o}}^{[1]}(r, t)$, where

$$
\begin{aligned}
z_{\mathfrak{o}}^{[1]}(r, t) & :=\int_{(x, \widetilde{\mathbf{y}}) \in \mathfrak{p}^{(9)}}|x|_{\mathfrak{p}}^{t}\left\|\left\{\widetilde{y}_{1}, \widetilde{y}_{2}, \widetilde{y}_{3}, x\right\}\right\|_{\mathfrak{p}}^{2 r} d \mu(x, \widetilde{\mathbf{y}}) \\
& =\sum_{(l, n) \in \mathbb{N}^{2}}\left(1-q^{-1}\right) q^{-n} M_{l} q^{-n t-2 \min \{l, n\} r}
\end{aligned}
$$

with

$$
M_{l}=\mu\left(\left\{\widetilde{\mathbf{y}} \in \mathfrak{p}^{(8)} \mid \max \left\{\left|\widetilde{y}_{1}\right|_{\mathfrak{p}},\left|\widetilde{y}_{2}\right|_{\mathfrak{p}},\left|\widetilde{y}_{3}\right|_{\mathfrak{p}}\right\}=q^{-l}\right\}\right)=\left(1-q^{-3}\right) q^{-3 l-5}
$$

This gives

$$
z_{\mathfrak{o}}^{[1]}(r, t)=\left(1-q^{-1}\right)\left(1-q^{-3}\right) q^{-5} \sum_{(l, n) \in \mathbb{N}^{2}} q^{(-1-t) n-3 l-2 r \min \{l, n\}} .
$$

Using the fact that

$$
\sum_{(l, n) \in \mathbb{N}^{2}} X_{1}^{l} X_{2}^{n} X_{3}^{\min \{l, n\}}=\frac{X_{1} X_{2} X_{3}\left(1-X_{1} X_{2}\right)}{\left(1-X_{1} X_{2} X_{3}\right)\left(1-X_{1}\right)\left(1-X_{2}\right)}
$$

we obtain further that

$$
z_{\mathfrak{o}}^{[1]}(r, t)=\frac{q^{-9-2 r-t}\left(1-q^{-4-t}\right)\left(1-q^{-1}\right)}{\left(1-q^{-4-2 r-t}\right)\left(1-q^{-1-t}\right)} .
$$


By (6.3) we have

$$
\begin{aligned}
z_{\mathfrak{o}}(r, t) & =\left(q^{8}-1-\left(\left|\overline{\mathcal{V}_{1}}\left(\mathbb{F}_{p}\right)\right|-1\right)\right) z_{\mathfrak{o}}^{[0]}(r, t)+\left(\left|\overline{\mathcal{V}_{1}}\left(\mathbb{F}_{p}\right)\right|-1\right) z_{\mathfrak{o}}^{[1]}(r, t) \\
& =\left(q^{8}-1-\left(q^{2}+q+1\right)^{2}(q-1)\right) z_{\mathfrak{o}}^{[0]}(r, t)+\left(q^{2}+q+1\right)^{2}(q-1) z_{\mathfrak{o}}^{[1]}(r, t),
\end{aligned}
$$

and a straightforward computation, together with equation (3.4), reveals that

$$
\begin{aligned}
\mathcal{P}_{\mathcal{R}, \mathfrak{o}}(s+2) & =1+\left(1-q^{-1}\right)^{-1} z_{\mathfrak{o}}(-s / 2-1,3 s-3) \\
& =\frac{q^{5}+\left(-q-q^{2}-q^{3}+q^{4}+q^{5}\right) q^{-2 s}+\left(1+q-q^{2}-q^{3}-q^{4}\right) q^{-3 s}+q^{-5 s}}{q^{5}\left(1-q^{1-2 s}\right)\left(1-q^{2-3 s}\right)} .
\end{aligned}
$$

Multiplying the last expression by $q^{8 m}$ we obtain, by Proposition 3.1, an explicit formula for $\zeta_{\mathrm{SL}_{3}^{m}(\mathfrak{o})}(s)$, as stated in Theorem $\mathrm{E}$. Note that in this particular instance the functional equation established in Theorem $\mathrm{A}$ follows from the fact that

$$
\left.\mathcal{P}_{\mathcal{R}, \mathfrak{o}}(s+2)\right|_{q \rightarrow q^{-1}}=q^{8} \mathcal{P}_{\mathcal{R}, \mathfrak{o}}(s+2),
$$

which can be easily verified directly. The explicit formulae for the zeta functions of groups of the form $\mathrm{SL}_{3}^{m}(\mathfrak{o})$, o an unramified extension of $\mathbb{Z}_{3}$, which are provided in [4] show that such functional equations are not satisfied for these groups.

Anticipating our computations in Section 7.3, we record an alternative formula for $\zeta_{\mathrm{SL}_{3}^{1}(\mathfrak{o})}(s)$, in the case where $m=1$ is permissible for $\mathfrak{s l}_{3}(\mathfrak{o})$. Recalling the notion of regular and irregular representations introduced above, we have

$$
\zeta_{\mathrm{SL}_{3}^{1}(\mathfrak{o})}(s)=1+\zeta_{\mathrm{SL}_{3}^{1}(\mathfrak{o})}^{\mathrm{reg}}(s)+\zeta_{\mathrm{SL}_{3}^{1}(\mathfrak{o})}^{\mathrm{irreg}}(s),
$$

where the three summands $1, \zeta_{\mathrm{SL}_{3}^{1}(\mathfrak{o})}^{\mathrm{reg}}(s)$ and $\zeta_{\mathrm{SL}_{3}^{1}(\mathfrak{o})}^{\text {irreg }}(s)$ enumerate the trivial, the regular and the irregular representations of $\mathrm{SL}_{3}^{1}(\mathfrak{o})$ respectively. Our computations above yield the following formulae for these summands:

$$
\begin{aligned}
\zeta_{\mathrm{SL}_{3}^{1}(\mathfrak{o})}^{\mathrm{reg}}(s) & =\left(q^{8}-1-\left(q^{2}+q+1\right)^{2}(q-1)\right)\left(1+q^{8}\left(1-q^{-1}\right)^{-1} z_{\mathfrak{o}}^{[0]}(-(s+2) / 2,3 s-3)\right) \\
& =\left(q^{8}-1-\left(q^{2}+q+1\right)^{2}(q-1)\right) \frac{1}{1-q^{2-3 s}}, \\
\zeta_{\mathrm{SL}_{3}^{1}(\mathfrak{o})}^{\mathrm{ireg}}(s) & =\left(q^{2}+q+1\right)^{2}(q-1)\left(1+q^{8}\left(1-q^{-1}\right)^{-1} z_{\mathfrak{o}}^{[1]}(-(s+2) / 2,3 s-3)\right) \\
& =\left(q^{2}+q+1\right)^{2}(q-1) \frac{1-q^{1-2 s}-q^{2-3 s}+q^{4-2 s}}{\left(1-q^{1-2 s}\right)\left(1-q^{2-3 s}\right)} .
\end{aligned}
$$

In Section 7.3 the right-most factors on the right hand side will be referred to as series factors.

6.2. Principal congruence subgroups of $\mathrm{SU}_{3}(\mathfrak{O}, \mathfrak{o})$. Recall that $\mathfrak{O} \mid \mathfrak{o}$ denotes an unramified quadratic extension with non-trivial automorphism $\sigma$, and that we assume $p \neq 3$. In this section we prove the second part of Theorem $\mathrm{E}$; we compute explicit formulae for the representation zeta functions of the principal congruence subgroups $\mathrm{SU}_{3}^{m}(\mathfrak{O}, \mathfrak{o})$, where $m$ is permissible for $\mathfrak{s u}_{3}(\mathfrak{O}, \mathfrak{o})$. We need to compute the integral (3.5) over $\mathfrak{p} \times W(\mathfrak{o})$, where $W(\mathfrak{o})=\left(\mathfrak{o}^{8}\right)^{*} \cong \operatorname{Hom}_{\mathfrak{o}}\left(\mathfrak{s u}_{3}(\mathfrak{O}, \mathfrak{o}), \mathfrak{o}\right)^{*}$. We make use of our computations for the principal congruence subgroups of $\mathrm{SL}_{3}(\mathfrak{O})$ in Section 6.1. Our argument 
is based on the commutative diagram

$$
\begin{aligned}
& \mathfrak{g l}_{3}(\mathfrak{K}) \cong \mathfrak{K} \otimes_{\mathfrak{k}} \mathfrak{g u}_{3}(\mathfrak{K}, \mathfrak{k}) \stackrel{\widetilde{\beta}}{\longrightarrow} \mathfrak{s l}_{3}(\mathfrak{K}) \cong \mathfrak{K} \otimes_{\mathfrak{k}} \mathfrak{s u}_{3}(\mathfrak{K}, \mathfrak{k}) \\
& \uparrow \text { inclusion } \uparrow \text { inclusion } \\
& \mathfrak{g u}_{3}(\mathfrak{K}, \mathfrak{k}) \quad \stackrel{\beta}{\longrightarrow} \quad \mathfrak{s u}_{3}(\mathfrak{K}, \mathfrak{k})
\end{aligned}
$$

where the map $\widetilde{\beta}$ and its restriction $\beta$ map an element $\mathbf{x}$ to $\widetilde{\beta}(\mathbf{x})=\mathbf{x}-\operatorname{Tr}(\mathbf{x}) / 3 \cdot \operatorname{Id}_{3}$. Note that the trace of an element $\mathbf{x} \in \mathfrak{g} \mathfrak{u}_{3}(\mathfrak{K}, \mathfrak{k})$ lies in $\mathfrak{g u _ { 1 }}(\mathfrak{K}, \mathfrak{k})$ so that $\beta(\mathbf{x})$ lies in $\mathfrak{s u}_{3}(\mathfrak{K}, \mathfrak{k})$, as indicated. Since $\mathfrak{s l}_{3}(\mathfrak{K}) \cong \mathfrak{K} \otimes_{\mathfrak{k}} \mathfrak{s u}_{3}(\mathfrak{K}, \mathfrak{k})$, we have for every $\mathbf{x} \in \mathfrak{s u}_{3}(\mathfrak{K}, \mathfrak{k})$,

$$
\operatorname{dim}_{\mathfrak{k}} \mathrm{C}_{\mathfrak{s u} 3(\mathfrak{K}, \mathfrak{k})}(\mathbf{x})=\operatorname{dim}_{\mathfrak{K}} \mathrm{C}_{\mathfrak{s} \mathfrak{l}_{3}(\mathfrak{K})}(1 \otimes \mathbf{x}) .
$$

We now fix an $\mathfrak{o}$-basis for $\mathfrak{O}$. For $p>3$ we may, for instance, choose $(1, \sqrt{\delta})$, where $\delta \in \mathfrak{o}$ is not a square modulo $\mathfrak{p}$. For any $\mathfrak{K}$-variety $\mathcal{V}$ let $\operatorname{res}_{\mathfrak{K} \mid \mathfrak{k}}(\mathcal{V})$ denote the $\mathfrak{k}$-variety obtained from $\mathcal{V}$ by restriction of scalars with respect to this basis, considered as a $\mathfrak{k}-$ basis for $\mathfrak{K}$. Thus the set of $\mathfrak{O}$-points $\mathcal{V}(\mathfrak{O})$ is in natural correspondence with the set of $\mathfrak{o}$-points $\operatorname{res}_{\mathfrak{K} \mid \mathfrak{k}}(\mathcal{V})(\mathfrak{o})$. Given $\mathbf{x} \in \mathcal{V}(\mathfrak{O})$ or $\mathbf{x} \in \mathcal{V}(\mathfrak{K})$, we write $\operatorname{res}_{\mathfrak{K} \mid \mathfrak{k}}(\mathbf{x})$ to denote the corresponding point in $\operatorname{res}_{\mathfrak{K} \mid \mathfrak{k}}(\mathcal{V})(\mathfrak{o})$ or $\operatorname{res}_{\mathfrak{K} \mid \mathfrak{k}}(\mathcal{V})(\mathfrak{k})$, respectively.

With this notation, the inclusions $\mathfrak{g u}_{3}(\mathfrak{K}, \mathfrak{k}) \subseteq \mathfrak{g l}_{3}(\mathfrak{K})$ and $\mathfrak{s u}_{3}(\mathfrak{K}, \mathfrak{k}) \subseteq \mathfrak{s l}_{3}(\mathfrak{K})$ admit natural interpretations at the level of algebraic varieties. Indeed, $\mathfrak{g u}_{3}(\mathfrak{K}, \mathfrak{k})$ can be regarded as a 9-dimensional $\mathfrak{k}$-linear subspace of the set of $\mathfrak{k}$-points $\operatorname{res}_{\mathfrak{K} \mid \mathfrak{k}}\left(\mathfrak{g l}_{3}\right)(\mathfrak{k})$ of the $\mathfrak{k}$-variety $\operatorname{res}_{\mathfrak{K} \mid \mathfrak{k}}\left(\mathfrak{g l}_{3}\right) \cong \mathbb{A}^{18}$. We denote, in the sequel, by $\mathfrak{g u}_{3}(\mathfrak{K}, \mathfrak{k})$ both the $\mathfrak{k}$-variety and its $\mathfrak{k}$-rational points; similar remarks apply to $\mathfrak{s u}_{3}(\mathfrak{K}, \mathfrak{k})$.

After these preparations, we consider the $\mathfrak{k}$-variety $\mathcal{V}_{1}$ of irregular elements in $\mathfrak{s u}_{3}(\mathfrak{K}, \mathfrak{k})$. Let $\widetilde{\mathcal{V}}_{1}$ denote the $\mathfrak{K}$-variety of irregular elements in the $\mathfrak{K}$-variety $\mathfrak{s l}_{3}$. Equation (6.7) says that

$$
\mathcal{V}_{1}(\mathfrak{k})=\operatorname{res}_{\mathfrak{K} \mid \mathfrak{k}}\left(\widetilde{\mathcal{V}}_{1}\right)(\mathfrak{k}) \cap \mathfrak{s u}_{3}(\mathfrak{K}, \mathfrak{k})=\left\{\mathbf{x} \in \widetilde{\mathcal{V}}_{1}(\mathfrak{K}) \mid \mathbf{x}^{\circ}+\mathbf{x}=0\right\} .
$$

We know, from the discussion in Section 6.1, that $\widetilde{\mathcal{V}}_{1}$ may be realised as the image of the $\mathfrak{K}$-variety $\widetilde{\mathcal{D}}_{1}$ of matrices of rank at most 1 under the projection map $\widetilde{\beta}$ and that the restricted map $\left.\widetilde{\beta}\right|_{\widetilde{D}_{1}}$ is injective. The next lemma shows that $\mathcal{V}_{1}(\mathfrak{k})$ is the image under $\beta$ of the $\mathfrak{k}$-points of the $\mathfrak{k}$-variety

$$
\mathcal{D}_{1}:=\operatorname{res}_{\mathfrak{K} \mid \mathfrak{k}}\left(\widetilde{\mathcal{D}}_{1}\right) \cap \mathfrak{g u}_{3}(\mathfrak{K}, \mathfrak{k}) .
$$

Lemma 6.1. Let $\mathbf{x} \in \widetilde{\mathcal{D}}_{1}(\mathfrak{K})$ such that $\widetilde{\beta}(\mathbf{x}) \in \mathfrak{s u}_{3}(\mathfrak{K}, \mathfrak{k})$. Then $\mathbf{x} \in \mathfrak{g u}_{3}(\mathfrak{K}, \mathfrak{k})$.

Proof. We need to show that $\mathbf{x}^{\circ}=-\mathbf{x}$. Because $\widetilde{\beta}$ is injective on $\widetilde{\mathcal{D}}_{1}$, the claim follows from the observation that $\widetilde{\mathcal{D}}_{1}$ is invariant under the operation $\mathbf{x} \mapsto \mathbf{x}^{\circ}$ and that

$$
\widetilde{\beta}\left(\mathbf{x}^{\circ}\right)=\widetilde{\beta}(\mathbf{x})^{\circ}=-\widetilde{\beta}(\mathbf{x})^{\circ}=\widetilde{\beta}\left(-\mathbf{x}^{\circ}\right) \text {. }
$$

We may consider $\beta$ and, by restriction of scalars, $\widetilde{\beta}$ as morphisms of $\mathfrak{k}$-varieties. As $p \neq 3$, the morphism $\widetilde{\beta}: \mathfrak{g l}_{3}(\mathfrak{O}) \rightarrow \mathfrak{s l}_{3}(\mathfrak{O})$ induces a morphism $\widetilde{\beta}_{\mathfrak{p}}: \operatorname{res}_{\mathfrak{K} \mid \mathfrak{k}}\left(\mathfrak{g l}_{3}\right)(\mathfrak{o}) \rightarrow$ $\operatorname{res}_{\mathfrak{K} \mid \mathfrak{e}}\left(\mathfrak{s l}_{3}\right)(\mathfrak{o})$ with good reduction modulo $\mathfrak{p}$. As we noted in Section 6.1, the map $\left.\widetilde{\beta}\right|_{\widetilde{\mathcal{D}}_{1}(\mathfrak{O})}: \widetilde{\mathcal{D}}_{1}(\mathfrak{O}) \rightarrow \widetilde{\mathcal{V}}_{1}(\mathfrak{O})$ is a bijection. Using Lemma 6.1 one sees that $\left.\beta_{\mathfrak{p}}\right|_{\mathcal{D}_{1}(\mathfrak{o})}$ : $\mathcal{D}_{1}(\mathfrak{o}) \rightarrow \mathcal{V}_{1}(\mathfrak{o})$ is a bijection.

Proposition 6.2. The $\mathfrak{k}$-variety $\mathcal{D}_{1} \backslash\{\mathbf{0}\}$ is smooth and 5-dimensional. It has good reduction modulo $\mathfrak{p}$, which has $\left(q^{4}+q^{2}+1\right)(q-1) \mathbb{F}_{q}$-rational points. 
Proof. We consider the surjective 'Segre map' of $\mathfrak{K}$-varieties

$$
\operatorname{seg}: \mathbb{A}^{3} \times \mathbb{A}^{3} \rightarrow \widetilde{\mathcal{D}}_{1}, \quad(\mathbf{a}, \mathbf{b}) \mapsto \mathbf{a}^{\mathrm{t}} \cdot \mathbf{b}
$$

and note that, for all $\mathbf{a}, \mathbf{b} \in \mathbb{A}^{3}(\mathfrak{K})$ with $\mathbf{x}:=\operatorname{seg}(\mathbf{a}, \mathbf{b}) \in \widetilde{\mathcal{D}}_{1}(\mathfrak{K}) \backslash\{\mathbf{0}\}$, we have $\left(\operatorname{seg}^{-1}(\mathbf{x})\right)(\mathfrak{K})=\left\{\left(\mu \mathbf{a}, \mu^{-1} \mathbf{b}\right) \mid \mu \in \mathfrak{K}^{*}\right\} \cong\left(\mathbb{A}^{1}\right)^{*}(\mathfrak{K})$. Recalling that $\sigma$ denotes the non-trivial Galois automorphism of $\mathfrak{K}$ over $\mathfrak{k}$, we first establish

$$
\mathcal{D}_{1}(\mathfrak{k})=\left\{\operatorname{res}_{\mathfrak{K} \mid \mathfrak{k}}(\operatorname{seg})\left(\mathbf{a}, \lambda \mathbf{a}^{\sigma}\right) \mid \mathbf{a} \in \mathbb{A}^{3}(\mathfrak{K}) \backslash\{\mathbf{0}\}, \lambda \in \mathfrak{g u}_{1}(\mathfrak{K}, \mathfrak{k})\right\} .
$$

The inclusion ' $\supseteq$ ' is clear. To prove the reverse inclusion ' $\subseteq$ ', let $\mathbf{x}=\mathbf{a}^{\mathrm{t}} \cdot \mathbf{b} \in \widetilde{\mathcal{D}}_{1}(\mathfrak{K}) \backslash\{\mathbf{0}\}$ such that $\operatorname{res}_{\mathfrak{K} \mid \mathfrak{k}}(\mathbf{x}) \in \mathfrak{g u}_{3}(\mathfrak{K}, \mathfrak{k})$. Then $\mathbf{x}^{\circ}=\left(\mathbf{b}^{\sigma}\right)^{\mathrm{t}} \cdot \mathbf{a}^{\sigma}$. Since $\mathbf{x}$ and $\mathbf{x}^{\circ}$ have the same row span, the vectors $\mathbf{b}$ and $\mathbf{a}^{\sigma}$ are proportional, say $\mathbf{b}=\lambda \mathbf{a}^{\sigma}$, where $\lambda \in \mathfrak{K}^{*}$. The equation $\mathbf{x}^{\circ}+\mathbf{x}=0$ implies that $\lambda^{\sigma}+\lambda=0$, i.e. that $\lambda \in \mathfrak{g u}_{1}(\mathfrak{K}, \mathfrak{k})^{*}$. This finishes the proof of (6.8). The same argument shows that

$$
\mathcal{D}_{1}(\mathfrak{o})^{*}:=\left\{\mathbf{x} \in \mathcal{D}_{1}(\mathfrak{o}) \mid \mathbf{x} \not \equiv_{\mathfrak{p}} \mathbf{0}\right\}=\left\{\operatorname{res}_{\mathfrak{K} \mid \mathfrak{k}}(\operatorname{seg})\left(\mathbf{a}, \lambda \mathbf{a}^{\sigma}\right) \mid \mathbf{a} \in\left(\mathfrak{O}^{3}\right)^{*}, \lambda \in \mathfrak{g} \mathfrak{u}_{1}(\mathfrak{O}, \mathfrak{o})^{*}\right\} .
$$

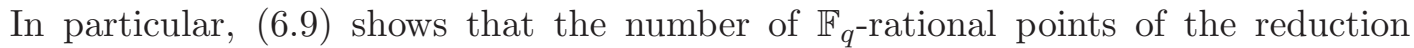
modulo $\mathfrak{p}$ of the pre-image $\left(\operatorname{res}_{\mathfrak{K} \mid \mathfrak{k}}(\operatorname{seg})\right)^{-1}\left(\mathcal{D}_{1}(\mathfrak{o})^{*}\right)$ in $\operatorname{res}_{\mathfrak{K} \mid \mathfrak{k}}\left(\mathbb{A}^{3} \times \mathbb{A}^{3}\right)$ is equal to

$$
\left|\left(\mathbb{A}^{3}\right)^{*}\left(\mathbb{F}_{q^{2}}\right)\right| \cdot\left|\mathfrak{g} \mathfrak{u}_{1}\left(\mathbb{F}_{q^{2}}, \mathbb{F}_{q}\right) \backslash\{0\}\right|=\left(q^{6}-1\right)(q-1) .
$$

Since the fibres of the projection seg are - with the exception of the fibre above zero - all isomorphic to $\left(\mathbb{A}^{1}\right)^{*}$, this implies that the number of $\mathbb{F}_{q}$-rational points of the reduction modulo $\mathfrak{p}$ of $\mathcal{D}_{1} \backslash\{\boldsymbol{0}\}$ is equal to $\left(q^{4}+q^{2}+1\right)(q-1)$.

To show that $\mathcal{D}_{1} \backslash\{\boldsymbol{0}\}$ is a smooth $\mathfrak{k}^{-v a r i e t y, ~ w e ~ s h o w ~ h o w ~ i t ~ c a n ~ b e ~ c o v e r e d ~ b y ~ o p e n ~}$ charts which are each isomorphic to the $\mathfrak{k}$-variety $\mathbb{A}^{4} \times\left(\mathbb{A}^{1}\right)^{*} \cong \operatorname{res}_{\mathfrak{K} \mid \mathfrak{e}}\left(\mathbb{A}^{2}\right) \times\left(\mathbb{A}^{1}\right)^{*}$. For $i \in\{1,2,3\}$ set

$$
\mathcal{D}_{1}^{[i]}:=\left\{\operatorname{res}_{\mathfrak{K} \mid \mathfrak{k}}(X) \mid X \in \widetilde{\mathcal{D}}_{1} \text { such that } \operatorname{res}_{\mathfrak{K} \mid \mathfrak{k}}(X) \in \mathcal{D} \text { and } X_{i i} \neq 0\right\} .
$$

Clearly, these three charts cover $\mathcal{D}_{1} \backslash\{\mathbf{0}\}$. It remains to show that each of the charts is isomorphic to $\operatorname{res}_{\mathfrak{K} \mid \mathfrak{k}}\left(\mathbb{A}^{2}\right) \times\left(\mathbb{A}^{1}\right)^{*}$. Without loss of generality we consider only $\mathcal{D}_{1}^{[1]}$. For ease of notation we will set up an isomorphism between $\mathfrak{k}$-rational points of varieties, which can easily be extended to an isomorphism of varieties.

We first deal with the case that $p \neq 2$. Since $\mathfrak{O}$ is unramified over $\mathfrak{o}$, we may write $\mathfrak{O}=\mathfrak{o}(\sqrt{\delta})=\mathfrak{o}+\mathfrak{o} \sqrt{\delta}$, where $\delta \in \mathfrak{o}$ is not a square modulo $\mathfrak{p}$. Writing $\xi:=\sqrt{\delta}$, we have $\mathfrak{g u} \mathfrak{u}_{1}(\mathfrak{K}, \mathfrak{k}) \backslash\{0\}=\mathfrak{k}^{*} \xi$. Hence $U:=\mathfrak{K}^{2} \times\left(\mathfrak{g u}_{1}(\mathfrak{K}, \mathfrak{k}) \backslash\{0\}\right) \cong\left(\operatorname{res}_{\mathfrak{G} \mid \mathfrak{k}}\left(\mathbb{A}^{2}\right) \times\left(\mathbb{A}^{1}\right)^{*}\right)(\mathfrak{k})$. Consider the morphism

$$
\varphi: U \rightarrow \mathcal{D}_{1}^{[1]}(\mathfrak{k}), \quad \varphi\left(\left(a_{2}, a_{3}, \lambda\right)\right) \mapsto \operatorname{res}_{\mathfrak{K} \mid \mathfrak{k}}\left(\operatorname{seg}\left(\left(1, a_{2}, a_{3}\right), \lambda\left(1, a_{2}^{\sigma}, a_{3}^{\sigma}\right)\right)\right) .
$$

Writing $a_{2}=a_{21}+a_{22} \xi, a_{3}=a_{31}+a_{32} \xi$ and $\lambda=c \xi$, where $a_{21}, \ldots, a_{32}, c \in \mathfrak{k}$ with $c \neq 0$, we can describe the effect of $\varphi$ implicitly in co-ordinates over $\mathfrak{k}$ :

$$
\varphi\left(\left(a_{2}, a_{3}, \lambda\right)\right)=\operatorname{res}_{\mathfrak{K} \mid \mathfrak{k}}(\mathbf{x}) \quad \text { where } \quad \mathbf{x}=c \xi\left(\begin{array}{ccc}
1 & a_{21}-a_{22} \xi & a_{31}-a_{32} \xi \\
a_{21}+a_{22} \xi & * & * \\
a_{31}+a_{32} \xi & * & *
\end{array}\right) .
$$

From this description we can deduce that $\varphi$ constitutes the desired isomorphism by extracting explicitly the inverse $\varphi^{-1}$ as a morphism over $\mathfrak{k}$,

$$
\varphi^{-1}: \mathcal{D}_{1}^{[1]}(\mathfrak{k}) \rightarrow U, \quad \operatorname{res}_{\mathfrak{K} \mid \mathfrak{k}}(\mathbf{x}) \mapsto\left(\frac{x_{12,2}}{x_{11,2}}-\frac{x_{12,1}}{x_{11,2} \delta} \xi, \frac{x_{13,2}}{x_{11,2}}-\frac{x_{13,1}}{x_{11,2} \delta} \xi, x_{11,2} \xi\right)
$$

where we write $x_{i j}=x_{i j, 1}+x_{i j, 2} \xi$. 
Suppose now that $\mathfrak{o}$ has residue characteristic $p=2$. In accordance with ArtinSchreier theory, we can write $\mathfrak{O}=\mathfrak{o}(\omega)=\mathfrak{o}+\mathfrak{o} \omega$, where $\omega=(1+\sqrt{\delta}) / 2$ with $\delta \in 1+4 \mathfrak{o}^{*}$, such that the reduction of $X^{2}-X-(\delta-1) / 4 \in \mathfrak{o}[X]$ modulo $\mathfrak{p}$ is irreducible over $\mathbb{F}_{q}=\mathfrak{o} / \mathfrak{p}$. As above, let $\sigma$ denote the non-trivial Galois automorphism of $\mathfrak{K}$ over $\mathfrak{k}$, where $\mathfrak{K}$ and $\mathfrak{k}$ denote the fields of fraction of $\mathfrak{O}$ and $\mathfrak{o}$. Then $(a+b \omega)^{\sigma}=(a+b)-b \boldsymbol{\omega}$ for $a, b \in \mathfrak{k}$, and hence $\mathfrak{g u}_{1}(\mathfrak{K}, \mathfrak{k})=\{a+b \omega \mid 2 a+b=0\}$.

To show that the chart $\mathcal{D}_{1}^{[1]}$ is isomorphic to $\operatorname{res}_{\mathfrak{K} \mid \mathfrak{k}}\left(\mathbb{A}^{2}\right) \times\left(\mathbb{A}^{1}\right)^{*}$, one defines as before the morphism $\varphi: U \rightarrow \mathcal{D}_{1}^{[1]}(\mathfrak{k})$. Writing $a_{2}=a_{21}+a_{22} \omega, a_{3}=a_{31}+a_{32} \omega$ and $\lambda=$ $c(1-2 \omega)$, where $a_{21}, \ldots, a_{32}, c \in \mathfrak{k}$ with $c \neq 0$, one describes $\varphi$ in co-ordinates and extracts explicitly the inverse $\varphi^{-1}$ as a morphism over $\mathfrak{k}$. For instance, one recovers $a_{2}=a_{21}+a_{22} \omega$ by means of the formulae

$$
a_{22}=\frac{x_{12,1}-x_{21,1}}{x_{11,1} \delta} \quad \text { and } \quad a_{21}=\frac{x_{21,1}}{x_{11,1}}+\frac{(\delta-1) a_{22}}{2},
$$

where we write $x_{i j}=x_{i j, 1}+x_{i j, 2} \omega$.

Proposition 6.2 allows us to compute the representation zeta functions of the principal congruence subgroups $\mathrm{SU}_{3}^{m}(\mathfrak{O}, \mathfrak{o})$, analogously to our work in Section 6.1. Indeed, for every permissible $m$ we obtain formulae analogous to (6.5) and (6.6), with $\left(q^{2}+q+1\right)^{2}$ replaced by $q^{4}+q^{2}+1$. A short computation yields the explicit formulae stated in Theorem E

\section{Abscissae of COnVergence for ARIthmetic groups of type $A_{2}$}

In this section we prove Theorem $\mathrm{C}$. Prerequisites about the structure of algebraic groups of type $A_{2}$ and their arithmetic subgroups are collected in Appendix $\mathrm{A}$. Our general strategy is as follows. In Section 7.3 we deal in detail with arithmetic groups of type ${ }^{1} A_{2}$ (inner forms) and in Section 7.4 we describe the necessary modifications for treating groups of type ${ }^{2} A_{2}$ (outer forms). We show that, in fact, it suffices to study infinite Euler products of representation zeta functions associated to compact $p$-adic analytic groups of the form $\mathrm{SL}_{3}(\mathfrak{o})$ and, in the case of outer forms, also $\mathrm{SU}_{3}(\mathfrak{O}, \mathfrak{o})$. In order to prove that the abscissae of convergence of such Euler products are equal to 1, we produce via Clifford theory suitable approximations of the local Euler factors. These approximations allow us to control the analytic properties of the original products. The relevant group theoretic tools for the 'approximative Clifford theory' are prepared in Section 7.2. The approximations of the local Euler factors are designed to remove the need to decide whether or not characters on principal congruence subgroups - which we dealt with in Section [6 - are extendable to their respective inertia groups. We manufacture the approximative Dirichlet series according to a finite case distinction, reflecting aspects of the adjoint actions of the finite groups $\mathrm{GL}_{3}\left(\mathbb{F}_{q}\right)$ and $\mathrm{GU}_{3}\left(\mathbb{F}_{q^{2}}, \mathbb{F}_{q}\right)$ on the finite Lie algebras $\mathfrak{s l}_{3}\left(\mathbb{F}_{q}\right)$ and $\mathfrak{s u}_{3}\left(\mathbb{F}_{q^{2}}, \mathbb{F}_{q}\right)$, respectively. Relevant data of these finite actions, including centralisers and the numbers and sizes of orbits of various types, are collected in Tables 7.1 and $7.2\left(\right.$ for $\mathfrak{s l}_{3}\left(\mathbb{F}_{q}\right)$ ) and Tables 7.3 and $7.4\left(\right.$ for $\mathfrak{s u}_{3}\left(\mathbb{F}_{q^{2}}, \mathbb{F}_{q}\right)$ ). The tables are derived in Appendices B and C, respectively. Section 7.1 contains a few elementary facts and definitions which we need for our approximations of Dirichlet series.

Remark 7.1. In [3], we give explicit formulae for the representation zeta functions of groups of the form $\mathrm{SL}_{3}(\mathfrak{o})$. This allows for an alternative proof of Theorem $\mathrm{C}$ for groups of type ${ }^{1} A_{2}$ and stronger analytic results in this case. We also show in 3 , that irreducible 
characters of the principal congruence subgroups $\mathrm{SL}_{3}^{1}(\mathfrak{o})$ are extendable to their inertia groups in $\mathrm{SL}_{3}(\mathfrak{o})$. We anticipate that the arguments in the present paper will serve as a template for algebraic groups where this may either not be the case, not effectively decidable or where it is simply not practical to produce an explicit analysis.

7.1. Dirichlet generating functions. Consider Dirichlet generating functions $\xi(s)=$ $\sum_{n \in \mathbb{N}} a_{n} n^{-s}$ and $\eta(s)=\sum_{n \in \mathbb{N}} b_{n} n^{-s}$, encoding sequences $\left(a_{n}\right)_{n \in \mathbb{N}}$ and $\left(b_{n}\right)_{n \in \mathbb{N}}$ of nonnegative integers. We write $\xi(s) \ll \eta(s)$ if $\sum_{n=1}^{N} a_{n} \leq \sum_{n=1}^{N} b_{n}$ for all $N \in \mathbb{N}$. Recall that, if the sequence $\left(a_{n}\right)$ contains infinitely many non-zero terms, the abscissa of convergence of $\xi(s)$ is equal to $\lim \sup _{N \rightarrow \infty} \log \left(\sum_{n=1}^{N} a_{n}\right) / \log (N)$. Thus we observe that $\xi(s) \ll \eta(s)$ implies that the abscissa of convergence of $\xi(s)$ is less than or equal to the abscissa of convergence of $\eta(s)$. We require the following straightforward lemma.

Lemma 7.2. Let $\xi(s)$ and $\eta(s)$ be Dirichlet generating functions which admit product decompositions $\xi(s)=\prod_{i \in I} \xi_{i}(s)$ and $\eta(s)=\prod_{i \in I} \eta_{i}(s)$ over a countable index set $I$ in the ring of all Dirichlet generating functions. Suppose that $\xi_{i}(s) \ll \eta_{i}(s)$ for each $i \in I$.

Then $\xi(s) \ll \eta(s)$; in particular the abscissa of convergence of $\xi(s)$ is less than or equal to the abscissa of convergence of $\eta(s)$.

Proof. If $\prod_{j \in J} \xi_{j}(s) \ll \prod_{j \in J} \eta_{j}(s)$ for every finite subset $J \subseteq I$, then taking limits yields $\xi(s) \ll \eta(s)$. Hence we may assume that $I$ is finite, and by induction on $|I|$ it is enough to consider the case $I=\{1,2\}$.

It is convenient to introduce notation which puts the given generating functions in a more general setting. For $i \in\{1,2\}$, we write $\xi_{i}(s)=\sum_{x \in X_{i}} \operatorname{deg}(x)^{-s}$ and $\eta_{i}(s)=$ $\sum_{y \in Y_{i}} \operatorname{deg}(y)^{-s}$, where $X_{i}$ and $Y_{i}$ are countable sets and the degree maps deg: $X_{i} \rightarrow$ $\mathbb{N}$ and $\operatorname{deg}: Y_{i} \rightarrow \mathbb{N}$ have finite fibres. Put $\mathbf{X}:=X_{1} \times X_{2}$ and define $\operatorname{deg}(\mathbf{x}):=$ $\operatorname{deg}\left(x_{1}\right) \operatorname{deg}\left(x_{2}\right)$ for $\mathbf{x}=\left(x_{1}, x_{2}\right) \in \mathbf{X}$. Similarly, we put $\mathbf{Y}:=Y_{1} \times Y_{2}$ and $\operatorname{deg}(\mathbf{y}):=$ $\operatorname{deg}\left(y_{1}\right) \operatorname{deg}\left(y_{2}\right)$ for $\mathbf{y}=\left(y_{1}, y_{2}\right) \in \mathbf{Y}$. Then

$$
\xi(s)=\xi_{1}(s) \xi_{2}(s)=\sum_{x_{1} \in X_{1}} \operatorname{deg}\left(x_{1}\right)^{-s} \sum_{x_{2} \in X_{2}} \operatorname{deg}\left(x_{2}\right)^{-s}=\sum_{\mathbf{x} \in \mathbf{X}} \operatorname{deg}(\mathbf{x})^{-s}
$$

and, similarly, $\eta(s)=\sum_{\mathbf{y} \in \mathbf{Y}} \operatorname{deg}(\mathbf{y})^{-s}$.

For $i \in\{1,2\}$, the condition $\xi_{i}(s) \ll \eta_{i}(s)$ is equivalent to the existence of an injective map $\iota_{i}: X_{i} \rightarrow Y_{i}$ such that $\operatorname{deg}(x) \leq \operatorname{deg}\left(\iota_{i}(x)\right)$ for all $x \in X_{i}$. Clearly, the product map $\iota: X_{1} \times X_{2} \rightarrow Y_{1} \times Y_{2},\left(x_{1}, x_{2}\right) \rightarrow\left(\iota_{1}\left(x_{1}\right), \iota_{2}\left(x_{2}\right)\right)$ is injective and $\operatorname{deg}(\mathbf{x}) \leq \operatorname{deg}(\iota(\mathbf{x}))$ for all $\mathbf{x} \in X_{1} \times X_{2}$. In conjunction with (17.1) and the analogous description of $\eta(s)$, this implies that $\xi(s) \ll \eta(s)$.

7.2. Clifford theory. In this section we explain how Clifford theory can be used to compute precisely or approximatively the representation zeta function of a FAb compact $p$-adic analytic group $G$ from the representation zeta function of a saturable normal pro- $p$ subgroup $N$, whose irreducible characters can be described by the Kirillov orbit method. For instance, in Section 7.3 the theory will be applied to the concrete groups $G=\mathrm{SL}_{3}(\mathfrak{o})$ and $N=\mathrm{SL}_{3}^{1}(\mathfrak{o})$. A general and accessible reference for Clifford theory is [28, $\left.\S 19-22\right]$.

7.2.1. We begin in a more general setting and specialise to our specific cases of interest during the course of the discussion. Let $G$ be group and $N \unlhd G$ a normal subgroup of finite index. Clifford theory provides a connection between the irreducible characters of $G$ and those of $N$. To start with, there is a natural action of $G$ on $\widehat{N}=\operatorname{Irr}(N)$. The stabiliser of $\vartheta \in \widehat{N}$ under this action is the inertia group $I_{G}(\vartheta)$. Let $\operatorname{Irr}(G, \vartheta)$ denote the 
set of all irreducible characters $\rho$ of $G$ such that $\vartheta$ occurs as an irreducible constituent of the restricted character $\operatorname{res}_{N}^{G}(\rho)$. Then one has

$$
\operatorname{Irr}(G, \vartheta)=\left\{\operatorname{ind}_{I_{G}(\vartheta)}^{G}(\psi) \mid \psi \in \operatorname{Irr}\left(I_{G}(\vartheta), \vartheta\right)\right\} .
$$

For any $\rho \in \operatorname{Irr}(G, \vartheta)$, the irreducible components of $\operatorname{res}_{N}^{G}(\rho)$ are precisely the $G$ conjugates of $\vartheta$ and, in particular, their number is $\left|G: I_{G}(\vartheta)\right|$. We write

$$
\zeta_{G, \vartheta}(s):=\vartheta(1)^{s}\left|G: I_{G}(\vartheta)\right|^{s} \sum_{\rho \in \operatorname{Irr}(G, \vartheta)} \rho(1)^{-s}=\vartheta(1)^{s} \sum_{\psi \in \operatorname{Irr}\left(I_{G}(\vartheta), \vartheta\right)} \psi(1)^{-s} .
$$

If $N$ admits only finitely many irreducible characters of any given degree, it follows that

$$
\zeta_{G}(s)=\sum_{\rho \in \widehat{G}} \rho(1)^{-s}=\sum_{\vartheta \in \widehat{N}} \vartheta(1)^{-s} \cdot\left|G: I_{G}(\vartheta)\right|^{-1-s} \zeta_{G, \vartheta}(s) .
$$

Remark 7.3. In our applications of this equation, e.g. in Sections 7.3 and 7.4, the terms $\left|G: I_{G}(\vartheta)\right|^{-1-s} \zeta_{G, \vartheta}(s)$ appearing on the right hand side of (7.2) will be referred to as 'translation factors'.

In the special case where $\vartheta$ extends to an irreducible character $\hat{\vartheta}$ of $I_{G}(\vartheta)$, there is an effective description of the elements $\psi \in \operatorname{Irr}\left(I_{G}(\vartheta), \vartheta\right)$ : according to [28, Theorem 19.6] one has

$$
\operatorname{Irr}\left(I_{G}(\vartheta), \vartheta\right)=\left\{\hat{\vartheta} \varphi \mid \varphi \in \operatorname{Irr}\left(I_{G}(\vartheta) / N\right)\right\} \quad \text { and } \quad \zeta_{G, \vartheta}(s)=\zeta_{I_{G}(\vartheta) / N}(s) .
$$

There are several basic sufficient criteria for the extensibility of $\vartheta$. For instance, if $N$ is a finite $p$-group it suffices that a Sylow- $p$ subgroup of $I_{G}(\vartheta) / N$ is cyclic; see [28, Theorem 19.13].

Lemma 7.4. Let $G$ be a finite group, and let $N$ be a normal subgroup of $G$. Let $\vartheta \in \operatorname{Irr}(N)$ and set $I:=I_{G}(\vartheta)$. Let $q$ be a power of $p$. Suppose that $N$ is a p-group and that $\vartheta(1)$ is a power of $q$. Let $P / N$ be a Sylow p-subgroup of $I / N$.

(1) Suppose that $\vartheta$ extends to an irreducible character of $P$. Then $\vartheta$ extends to $I$ and $\zeta_{G, \vartheta}(s)=\zeta_{I / N}(s)$.

(2) Suppose that $\vartheta$ does not extend to $I$ and that the character degrees of $P$ are powers of $q$. Then $|P: N| \geq q^{2}$ and $\zeta_{G, \vartheta}(s) \ll\left\lfloor q^{-2}|I: N|\right\rfloor q^{-s}$.

Proof. (1) This follows from [28, Theorems 21.4 and 22.3].

(2) We first prove the second statement. Let $\psi_{1}, \ldots, \psi_{r}$ denote the irreducible constituents of the induced character $\operatorname{ind}_{N}^{I}(\vartheta)$ so that $\operatorname{ind}_{N}^{I}(\vartheta)=\sum_{i=1}^{r} e_{i} \psi_{i}$. We claim that (i) $r \leq q^{-2}|I: N|$ and (ii) $\psi_{i}(1) \geq q \vartheta(1)$ for all $i \in\{1, \ldots, r\}$. From this the claim about $\zeta_{G, \vartheta}(s)$ follows directly.

The proof of the inequality (ii) is straightforward. Let $i \in\{1, \ldots, r\}$. Since $\vartheta$ does not extend to $I$, it does not extend to $P$. But $\varphi:=\operatorname{res}_{P}^{I}\left(\psi_{i}\right)$ admits an irreducible constituent whose restriction to $N$ involves $\vartheta$. This shows that $\varphi(1)>\vartheta(1)$. Since $\varphi(1)$ and $\vartheta(1)$ are powers of $q$, we deduce that $\psi_{i}(1)=\varphi(1) \geq q \vartheta(1)$, as wanted.

It remains to prove the inequality (i). Observe that for each $i \in\{1, \ldots, r\}$ we have $\operatorname{res}_{N}^{I}\left(\psi_{i}\right)=e_{i} \vartheta$ so that $e_{i}=\psi_{i}(1) / \vartheta(1) \geq q$, using (ii). Therefore

$$
|I: N| \vartheta(1)=\left(\operatorname{ind}_{N}^{I}(\vartheta)\right)(1)=\sum_{i=1}^{r} e_{i} \psi_{i}(1) \geq r q^{2} \vartheta(1),
$$

again using (ii). Cancelling $\vartheta(1)$ and rearranging terms yields (i).

The claim $|P: N| \geq q^{2}$ follows from (i), if we replace both $G$ and $I$ by $P$. 
7.2.2. Now suppose that $G$ is a FAb compact $p$-adic analytic group and $N$ is a saturable normal pro- $p$ subgroup of $G$, whose irreducible characters can be described by the Kirillov orbit method. A central step in connecting $\widehat{G}=\operatorname{Irr}(G)$ with $\widehat{N}=\operatorname{Irr}(N)$ consists in computing the inertia groups $I_{G}(\vartheta)$ for $\vartheta \in \widehat{N}$. Let $\mathfrak{n}$ be the saturable $\mathbb{Z}_{p}$-Lie lattice associated to $N$. Then each $\vartheta \in \widehat{N}$ corresponds to an orbit $\Omega=\omega^{N}$ in the co-adjoint action of $N$ on $\widehat{\mathfrak{n}}=\operatorname{Irr}(\mathfrak{n})$. Thus $I_{G}(\vartheta)$ is the set-wise stabiliser of $\Omega$ under the co-adjoint action of $G$ on $\widehat{\mathfrak{n}}$, and

$$
I_{G}(\vartheta)=\mathrm{N}_{G}(\Omega)=\mathrm{C}_{G}(\omega) N .
$$

We now specialise to the situation which is most interesting to us. Let $k$ be an algebraic number field with ring of integers $\mathcal{O}$, and let $\Lambda$ be an $\mathcal{o}$-Lie lattice such that $k \otimes_{\mathcal{O}} \Lambda$ is a finite dimensional, semisimple $k$-Lie algebra. Let $\mathfrak{o}=\mathcal{O}_{v}$ be the completion of 0 at a non-archimedean place $v$ - to be restricted further during our discussion - with maximal ideal $\mathfrak{p}=\pi \mathfrak{o}$, lying above the rational prime $p$. Put $\mathfrak{g}:=\mathfrak{o} \otimes_{\mathfrak{o}} \Lambda$, and suppose that $\mathfrak{n}$ is the $\mathfrak{o}$-Lie lattice $\mathfrak{g}^{m}=\mathfrak{p}^{m} \mathfrak{g}$ for some $m \in \mathbb{N}_{0}$.

Observe that multiplication by $\pi^{-m}$ provides a $G$-equivariant isomorphism of $\mathfrak{o}$ modules $\mathfrak{n} \rightarrow \mathfrak{g}$, and a corresponding $G$-equivariant bijection $\widehat{\mathfrak{g}} \rightarrow \widehat{\mathfrak{n}}$. By means of these maps we may describe elements of $\widehat{N}$ by orbits in the co-adjoint action of $N$ on $\widehat{\mathfrak{g}}$ rather than $\widehat{\mathfrak{n}}$. From a computational point of view, it is convenient to work with the adjoint action of $G$ on $\mathfrak{g}$ rather than the co-adjoint action on $\widehat{\mathfrak{g}}$. In general, the necessary translation can be made by means of the Killing form $\kappa$ associated to the o-Lie lattice $\Lambda$. For simplicity we shall assume that $\Lambda=\mathcal{O} \otimes_{\mathbb{Z}} \Lambda_{0}$ arises from a $\mathbb{Z}$-Lie lattice $\Lambda_{0}$ of Chevalley type, associated to a simply-connected simple group scheme over $\mathbb{Z}$. Then the transition from the co-adjoint to the adjoint action can be carried out more effectively by using the normalised Killing form

$$
\kappa_{0}:=\left(2 h^{\vee}\right)^{-1} \kappa: \Lambda \times \Lambda \rightarrow \mathcal{O},
$$

where $h^{\vee}$ denotes the dual Coxeter number. Writing $d:=\operatorname{dim}_{k}\left(k \otimes_{\mathcal{O}} \Lambda\right)$, the bilinear form $\kappa_{0}$ can be represented by a structure matrix $B \in \operatorname{Mat}_{d}(\mathbb{Z})$ with respect to an o-basis of $\Lambda$ obtained from a $\mathbb{Z}$-basis of $\Lambda_{0}$; [22, Section 5]. The determinant of $B$ is a non-zero integer. For instance, for $\Lambda_{0}=\mathfrak{s l}_{n}$, the determinant of $B$ is equal to $n$. Excluding from our discussion those places $v$ which lie above primes $p$ dividing the determinant of $B$, the form $\kappa_{0}$ extends to a 'non-degenerate' form on the local $\mathfrak{o}$-Lie lattice $\mathfrak{g}$ : for every $x \in \mathfrak{g}^{*}$ there exists $y \in \mathfrak{g}$ such that $\kappa_{0}(x, y) \in \mathfrak{o}^{*}$.

Thus $\kappa_{0}$ induces a natural $G$-equivariant isomorphism of $\mathfrak{o}$-modules

$$
\iota_{0}: \mathfrak{g} \rightarrow \operatorname{Hom}_{\mathfrak{o}}(\mathfrak{g}, \mathfrak{o})^{*}, \quad x \mapsto \kappa_{0}(x, \cdot) .
$$

For each $n \in \mathbb{N}$ there is a natural surjective map from $\operatorname{Hom}_{\mathfrak{o}}(\mathfrak{g}, \mathfrak{o})^{*}$ onto $\operatorname{Irr}_{n}(\mathfrak{g}) \cong$ $\operatorname{Hom}_{\mathfrak{o}}\left(\mathfrak{g}, \mathfrak{o} / \mathfrak{p}^{n}\right)^{*}$, and these sets partition $\operatorname{Irr}(\mathfrak{g})$; cf. Lemma 2.4. Thus the normalised Killing form gives rise to a $G$-equivariant commutative diagram

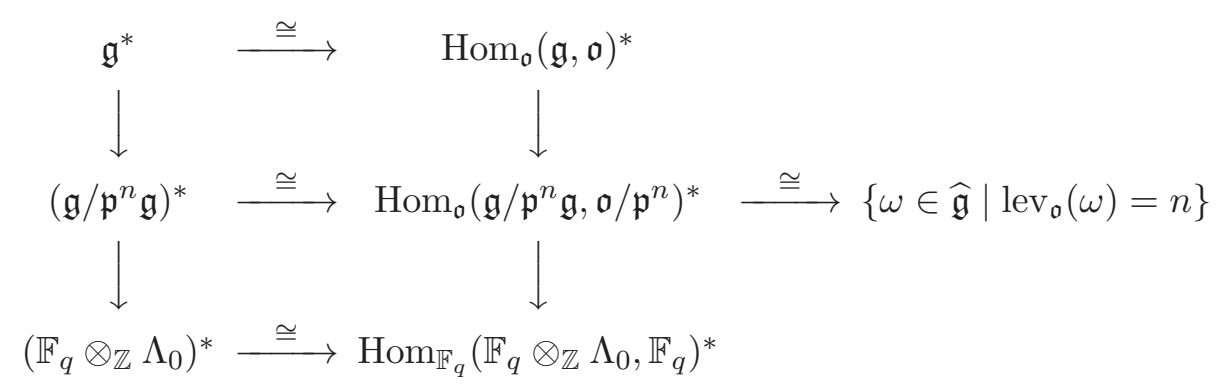


where the last row is obtained by reduction modulo $\mathfrak{p}$ and we have used the isomorphism $\mathfrak{o} / \mathfrak{p} \cong \mathbb{F}_{q}$.

Let us now restrict further to the case $m=1$, i.e. $\mathfrak{n}=\mathfrak{g}^{1}=\mathfrak{p g}$. Consider a non-trivial character $\vartheta \in \operatorname{Irr}(N)$, represented by an orbit $\omega^{N}$ in $\widehat{\mathfrak{g}} \cong \widehat{\mathfrak{n}}$. Put $n:=\operatorname{lev}_{\mathfrak{o}}(\omega)$, and make use of the diagram (7.4). We choose $w \in \operatorname{Hom}_{\mathfrak{o}}(\mathfrak{g}, \mathfrak{o})^{*}$ such that its image $\left.w\right|_{n}$ in $\operatorname{Hom}_{\mathfrak{o}}\left(\mathfrak{g} / \mathfrak{p}^{n} \mathfrak{g}, \mathfrak{o} / \mathfrak{p}^{n}\right)^{*}$ corresponds to $\omega$, and we write $\bar{w}$ for the image of $w$ in $\operatorname{Hom}_{\mathbb{F}_{q}}\left(\mathbb{F}_{q} \otimes_{\mathbb{Z}}\right.$ $\left.\Lambda_{0}, \mathbb{F}_{q}\right)^{*}$. By means of the horizontal isomorphisms, induced by the isomorphism $\iota_{0}$, we find $x,\left.x\right|_{n}=x+\mathfrak{p}^{n} \mathfrak{g}, \bar{x}$ corresponding to $w,\left.w\right|_{n}, \bar{w}$ respectively.

Since all the maps in (7.4) are $G$-equivariant, we conclude that $\mathrm{C}_{G}(\omega)=\mathrm{C}_{G}\left(x+\mathfrak{p}^{n} \mathfrak{g}\right)$. In general, $\mathrm{C}_{G}\left(x+\mathfrak{p}^{n} \mathfrak{g}\right) \leq \mathrm{C}_{G}(\bar{x})$ and this inclusion provides an upper bound for the size of the inertia group quotient. Under certain circumstances, one can prove that $\mathrm{C}_{G}(x) N=\mathrm{C}_{G}\left(x+\mathfrak{p}^{n} \mathfrak{g}\right) N=\mathrm{C}_{G}(\bar{x})$, hence

$$
I_{G}(\vartheta)=\mathrm{C}_{G}(\bar{x}) \quad \text { and } \quad I_{G}(\vartheta) / N \cong \mathrm{C}_{G / N}(\bar{x}) .
$$

The quotient group $G / N$ is typically (a subgroup of) a finite group of Lie type, and linear algebra allows one to describe the various possibilities for the centraliser $C:=\mathrm{C}_{G / N}(\bar{x})$. In the special cases of interest to us in the current paper, such an analysis is carried in Appendices B and C,

7.2.3. Let $n \in \mathbb{N}$. We conclude the Section 7.2 by recording some specific results for the case where the completion of $\Lambda$ leads to one of the $\mathfrak{o}$-Lie lattices $\mathfrak{s l}_{n}(\mathfrak{o})$ and $\mathfrak{s u}_{n}(\mathfrak{O}, \mathfrak{o})$. Here and in the following we use the notation introduced at the beginning of Section 6 , We fix an unramified quadratic extension $\mathfrak{O} \mid \mathfrak{o}$ of compact discrete valuation rings of characteristic 0. The non-trivial Galois automorphism of the corresponding extension $\mathfrak{K} \mid \mathfrak{k}$ of fields of fractions is denoted by $\sigma$. The standard involution on $\operatorname{Mat}_{n}(\mathfrak{K})$ is denoted by $\circ$. As $\mathfrak{O}$ is unramified over $\mathfrak{o}$, the uniformiser $\pi$ of $\mathfrak{o}$ also uniformises $\mathfrak{O}$.

The results in this section allow one to pin down various inertia group quotients in the context of Clifford theory for the group extensions $\mathrm{SL}_{n}^{1}(\mathfrak{o}) \unlhd \mathrm{SL}_{n}(\mathfrak{o})$ and $\mathrm{SU}_{n}^{1}(\mathfrak{O}, \mathfrak{o}) \unlhd$ $\mathrm{SU}_{n}(\mathfrak{O}, \mathfrak{o})$; for $n=3 \mathrm{such}$ an analysis is carried out explicitly in Sections 7.3 and 7.4 .

Lemma 7.5. Let $\mathbf{a}$ be an element of the $\mathfrak{o}$-order $\operatorname{Mat}_{n}(\mathfrak{o})$ of the central simple $\mathfrak{k}$-algebra $\operatorname{Mat}_{n}(\mathfrak{k})$. Writing - for reduction modulo $\mathfrak{p}$, suppose that the minimum polynomial of $\overline{\mathbf{a}}$ over $\mathbb{F}_{q}=\overline{\mathfrak{o}}$ is equal to the characteristic polynomial of $\overline{\mathbf{a}}$ in $\operatorname{Mat}_{n}\left(\mathbb{F}_{q}\right)$. Then one has

$$
\mathrm{C}_{\operatorname{Mat}_{n}\left(\mathbb{F}_{q}\right)}(\overline{\mathbf{a}})=\mathbb{F}_{q}[\overline{\mathbf{a}}] \quad \text { and } \quad \mathrm{C}_{\operatorname{Mat}_{n}(\mathfrak{o})}(\mathbf{a})=\mathfrak{o}[\mathbf{a}] ;
$$

consequently, $\mathrm{C}_{\operatorname{Mat}_{n}\left(\mathbb{F}_{q}\right)}(\overline{\mathbf{a}})=\overline{\mathrm{C}_{\operatorname{Mat}_{n}(\mathfrak{o})}(\mathbf{a})}$.

Proof. Let $f \in \mathfrak{o}[X]$ denote the characteristic polynomial of a over $\mathfrak{k}$. Since $\bar{f}$ is equal to the minimum polynomial of $\overline{\mathbf{a}}$ over $\mathbb{F}_{q}$, the polynomial $f$ is equal to the minimum polynomial of a over $\mathfrak{k}$. This implies that the natural $\mathbb{F}_{q}[\overline{\mathbf{a}}]$ - and $\mathfrak{o}[\mathbf{a}]$-modules $\bar{V}=\mathbb{F}_{q}^{n}$ and $V=\mathfrak{o}^{n}$ are each free of rank 1 . Choose a free generator $x$ of $V$ mapping onto a free generator $\bar{x}$ of $\bar{V}$. The centralisers featuring in our claim are identical to the endomorphism rings of the free modules $\bar{V}$ and $V$. By considering the images of $\bar{x}$ and $x$, these endomorphism rings are seen to be equal to $\mathbb{F}_{q}[\overline{\mathbf{a}}]$ and $\mathfrak{o}[\mathbf{a}]$, respectively.

Corollary 7.6. Let $\mathbf{x} \in \mathfrak{s l}_{n}(\mathfrak{o})^{*}$, and let $\overline{\mathbf{x}} \in \mathfrak{s l}_{n}\left(\mathbb{F}_{q}\right)^{*}$ denote the reduction of $\mathbf{x}$ modulo $\mathfrak{p}$. Suppose that $p \nmid n$ and that the minimum polynomial of $\overline{\mathbf{x}}$ over $\mathbb{F}_{q}$ coincides with the characteristic polynomial of $\overline{\mathbf{x}}$ in $\operatorname{Mat}_{n}\left(\mathbb{F}_{q}\right)$. Then one has

$$
\mathrm{C}_{\mathrm{SL}_{n}(\mathfrak{o})}(\overline{\mathbf{x}})=\mathrm{C}_{\mathrm{SL}_{n}(\mathfrak{o})}(\mathbf{x}) \mathrm{SL}_{n}^{1}(\mathfrak{o}) \text {. }
$$


Proof. Certainly, the group on the right hand side is contained in the group on the left hand side. We prove the reverse inclusion. Let $g \in \mathrm{C}_{\mathrm{SL}_{n}(\mathfrak{a})}(\overline{\mathbf{x}})$. Then Lemma 7.5 produces $h \in \mathrm{C}_{\operatorname{Mat}_{n}(\mathfrak{o})}(\mathbf{x})$ such that $h \equiv g$ modulo $\mathfrak{p}$. Observe that $\operatorname{det}(h) \in 1+\mathfrak{p}$ is an $n$-th power, because $p \nmid n$. Let $\lambda \in 1+\mathfrak{p}$ such that $\lambda^{n}=\operatorname{det}(h)^{-1}$. Then $\lambda h \in \mathrm{C}_{\mathrm{SL}_{n}(\mathfrak{o})}(\mathbf{x})$ such that $h \equiv g$ modulo $\mathfrak{p}$ or, equivalently, modulo $\mathrm{SL}_{n}^{1}(\mathfrak{o})$, as wanted.

Corollary 7.7. Let $\mathbf{x} \in \mathfrak{s u}_{n}(\mathfrak{O}, \mathfrak{o})^{*}$, and let $\overline{\mathbf{x}} \in \mathfrak{s u}_{n}\left(\mathbb{F}_{q^{2}}, \mathbb{F}_{q}\right)^{*}$ denote the reduction of $\mathbf{x}$ modulo $\mathfrak{P}$. Suppose that $p>2$ with $p \nmid n$ and that the minimum polynomial of $\overline{\mathbf{x}}$ over $\mathbb{F}_{q^{2}}$ coincides with the characteristic polynomial of $\mathbf{x}$ in $\operatorname{Mat}_{n}\left(\mathbb{F}_{q^{2}}\right)$. Then one has

$$
\mathrm{C}_{\mathrm{SU}_{n}(\mathfrak{O}, \mathfrak{o})}(\overline{\mathbf{x}})=\mathrm{C}_{\mathrm{SU}_{n}(\mathfrak{O}, \mathfrak{o})}(\mathbf{x}) \mathrm{SU}_{n}^{1}(\mathfrak{O}, \mathfrak{o}) .
$$

Proof. Again, the group on the right hand side is clearly contained in the group on the left hand side, and it remains to prove the reverse inclusion. Let $g \in \mathrm{C}_{\mathrm{SU}_{n}(\mathfrak{O}, \mathfrak{o})}(\overline{\mathbf{x}})$. By Lemma 7.5, we find $h_{1} \in \mathrm{C}_{\operatorname{Mat}_{n}(\mathfrak{O})}(\mathbf{x})$ such that $h_{1} \equiv g$ modulo $\mathfrak{P}$.

We observe that $h_{1}^{\circ} h_{1} \equiv 1$ modulo $\mathfrak{P}$, and we carry out a 'Hensel's Lemma type' argument to manufacture, inductively, a sequence $h_{i}, i \in \mathbb{N}$, in $\mathrm{C}_{\operatorname{Mat}_{n}(\mathfrak{O})}(\overline{\mathbf{x}})$ such that

$$
h_{i}^{\circ} h_{i} \equiv 1 \quad \text { and } \quad h_{i+1} \equiv h_{i} \quad \text { modulo } \mathfrak{P}^{i}
$$

which, in the limit, yields $h=\lim _{i \rightarrow \infty} h_{i} \in \mathrm{C}_{\mathrm{GU}_{n}(\mathfrak{O}, \mathfrak{o})}(\mathbf{x})$ such that $h \equiv g$ modulo $\mathfrak{P}$. Similarly as in the proof of Corollary 7.6, a modification by a scalar $\lambda \in \mathrm{GU}_{1}^{1}(\mathfrak{O}, \mathfrak{o})$ such that $\lambda^{n}=\operatorname{det}(h)^{-1}$ then yields the desired element $\lambda h \in \mathrm{C}_{\mathrm{SU}_{n}(\mathfrak{O}, \mathfrak{o})}(\mathbf{x})$ such that $h \equiv g$ modulo $\mathfrak{P}$ or, equivalently, modulo $\mathrm{SU}_{n}^{1}(\mathfrak{O}, \mathfrak{o})$.

Let $i \in \mathbb{N}$ and suppose we have determined $h_{1}, \ldots, h_{i} \in \mathrm{C}_{\operatorname{Mat}_{n}(\mathfrak{O})}(\mathbf{x})$ with the desired properties. Since $\mathbf{x}^{\circ}=-\mathbf{x}$, the centraliser $\mathrm{C}_{\operatorname{Mat}_{n}(\mathfrak{O})}(\mathbf{x})$ is invariant under the involution $\circ$. Recall that $\pi$ is a uniformiser for $\mathfrak{O}$, and put $h_{i+1}:=h_{i}+\pi^{i} y_{i}$ where

$$
y_{i}:=-2^{-1} \pi^{-i}\left(h_{i}^{\circ}\right)^{-1}\left(h_{i}^{\circ} h_{i}-1\right) \in \mathrm{C}_{\operatorname{Mat}_{n}(\mathfrak{O})}(\mathbf{x}) .
$$

Then $h_{i+1} \equiv h_{i}$ modulo $\mathfrak{P}^{i}$, and modulo $\mathfrak{P}^{i+1}$ one has

$$
\begin{aligned}
h_{i+1}^{\circ} h_{i+1}-1 & =\left(h_{i}^{\circ} h_{i}+\pi^{i}\left(h_{i}^{\circ} y_{i}+y_{i}^{\circ} h_{i}\right)+\pi^{2 i} y_{i}^{\circ} y_{i}\right)-1 \\
& \equiv\left(h_{i}^{\circ} h_{i}-1\right)+\pi^{i}\left(\left(h_{i}^{\circ} y_{i}\right)+\left(h_{i}^{\circ} y_{i}\right)^{\circ}\right) \\
& \equiv 0 .
\end{aligned}
$$

Lemma 7.8. Suppose that $p>2$ with $p \nmid n$. Let $H$ be a Sylow pro-p subgroup of $\operatorname{SL}_{n}(\mathfrak{o})$ or $\mathrm{SU}_{n}(\mathfrak{O}, \mathfrak{o})$, and accordingly let $N$ be equal to $\mathrm{SL}_{n}^{1}(\mathfrak{o})$ or $\mathrm{SU}_{n}^{1}(\mathfrak{O}, \mathfrak{o})$. Suppose that $H$ is saturable and that $N$ is saturable and potent. Let $\vartheta \in \operatorname{Irr}(N)$.

Then $I_{H}(\vartheta)$ corresponds to an $\mathfrak{o}$-Lie sublattice of the $\mathfrak{o}$-Lie lattice associated to $H$. In particular, $\left|I_{H}(\vartheta): N\right|$ is a power of $q=|\mathfrak{o}: \mathfrak{p}|$.

Proof. First suppose that $H$ is a Sylow pro- $p$ subgroup of $\operatorname{SL}_{n}(\mathfrak{o})$ and that $N=\operatorname{SL}_{n}^{1}(\mathfrak{o})$. Since $N$ is saturable and potent and since $p \nmid n$, we can apply the Kirillov orbit method and use the translation via the normalised Killing form in order to describe $I_{H}(\vartheta)$ in terms of the adjoint action. Let $\mathfrak{n}=\mathfrak{s l}_{n}^{1}(\mathfrak{o})$ denote the $\mathfrak{o}$-Lie lattice associated to $N$. In contrast to the discussion in Section 7.2.2, we consider directly the adjoint action on $\mathfrak{n}$, without shifting by a factor $\pi^{-1}$. Let $\omega^{N}$ be the co-adjoint orbit representing $\vartheta$. Then we have $I_{H}(\vartheta)=\mathrm{C}_{H}\left(x+\mathfrak{p}^{l} \mathfrak{n}\right) N$, where $l=\operatorname{lev}_{\mathfrak{o}}(\omega), x+\mathfrak{p}^{l} \mathfrak{n}$ corresponds to $\omega$ via the normalised Killing form, and the centraliser is formed with respect to the adjoint action. Since $H$ is saturable, it also corresponds to an $\mathfrak{o}$-Lie lattice $\mathfrak{h}$, and the centraliser $\mathrm{C}_{H}\left(x+\mathfrak{p}^{l} \mathfrak{n}\right)$ at the group level corresponds to the centraliser $\mathfrak{c}:=\mathrm{C}_{\mathfrak{h}}\left(x+\mathfrak{p}^{l} \mathfrak{n}\right)$ 
at the Lie lattice level. Since $\mathfrak{c}+\mathfrak{n}$ and $\mathfrak{n}$ are $\mathfrak{o}$-Lie sublattices of $\mathfrak{h}$, it follows that $\left|I_{H}(\vartheta): N\right|=|\mathfrak{c}+\mathfrak{n}: \mathfrak{n}|$ is a power of $q$.

The argument for the second case, i.e. $H$ a Sylow pro- $p$ subgroup of $\operatorname{SU}_{n}(\mathfrak{O}, \mathfrak{o})$ and $N=\mathrm{SU}_{n}^{1}(\mathfrak{O}, \mathfrak{o})$, is similar. But we need to justify that $I_{H}(\vartheta)$ can be described in terms of the adjoint action of $N$ on the $\mathfrak{o}$-Lie lattice $\mathfrak{n}=\mathfrak{s u}_{n}^{1}(\mathfrak{O}, \mathfrak{o})$. For this purpose we consider $\mathfrak{s u}_{n}(\mathfrak{O}, \mathfrak{o})$ as the subset of the $\mathfrak{O}$-Lie lattice $\mathfrak{s l}_{n}(\mathfrak{O})$, consisting of all elements $\mathbf{x}$ such that $\mathbf{x}^{\circ}+\mathbf{x}=0$. We claim that the normalised Killing form $\kappa_{0}$ on the $\mathfrak{O}$-Lie lattice $\mathfrak{s l}_{3}(\mathfrak{O})$ restricts to a non-degenerate bilinear form

$$
\left.\kappa_{0}\right|_{\mathfrak{s u}_{n}(\mathfrak{O}, \mathfrak{o}) \times \mathfrak{s u}_{n}(\mathfrak{O}, \mathfrak{o})}: \mathfrak{s u}_{n}(\mathfrak{O}, \mathfrak{o}) \times \mathfrak{s u}_{n}(\mathfrak{O}, \mathfrak{o}) \rightarrow \mathfrak{o} .
$$

Indeed, for any $\mathbf{x}, \mathbf{y} \in \mathfrak{s u}_{n}(\mathfrak{O}, \mathfrak{o})$ one computes $\kappa_{0}(\mathbf{x}, \mathbf{y})^{\sigma}=\kappa_{0}\left(\mathbf{x}^{\circ}, \mathbf{y}^{\circ}\right)=\kappa_{0}(-\mathbf{x},-\mathbf{y})=$ $\kappa_{0}(\mathbf{x}, \mathbf{y})$ which implies $\kappa_{0}(\mathbf{x}, \mathbf{y}) \in \mathfrak{o}$. This shows that $\left.\kappa_{0}\right|_{\mathfrak{s u}_{n}(\mathfrak{O}, \mathfrak{o})}$ maps into $\mathfrak{o}$. Of course, $\left.\kappa_{0}\right|_{\mathfrak{s u}_{n}(\mathfrak{O}, \mathfrak{o}) \times \mathfrak{s u}_{n}(\mathfrak{O}, \mathfrak{o})}$ is $\mathfrak{o}$-bilinear, and it remains to show that it is non-degenerate over $\mathfrak{o}$. Let $\mathbf{x} \in \mathfrak{s u}_{n}(\mathfrak{O}, \mathfrak{o})^{*}$. We need to produce $\mathbf{y} \in \mathfrak{s u}_{n}(\mathfrak{O}, \mathfrak{o})$ such that $\kappa_{0}(\mathbf{x}, \mathbf{y}) \in \mathfrak{o}^{*}$. Since $\kappa_{0}$ is non-degenerate on the ambient $\mathfrak{O}$-Lie lattice $\mathfrak{s l}_{n}(\mathfrak{O})$, we find $\mathbf{z} \in \mathfrak{s l}_{n}(\mathfrak{O})$ such that $\lambda:=\kappa_{0}(\mathbf{x}, \mathbf{z}) \in \mathfrak{O}^{*}$. Multiplying $\mathbf{z}$ by a suitable scalar, if necessary, we can arrange for $\lambda$ to be such that $\lambda+\lambda^{\sigma} \not \equiv 0$ modulo $\mathfrak{P}$. (This is equivalent to the condition that the reduction of $\lambda$ modulo $\mathfrak{P}$ does not have trace 0 in the residue field extension $\mathbb{F}_{q^{2}} \mid \mathbb{F}_{q}$.) Set $\mathbf{y}:=\mathbf{z}-\mathbf{z}^{\circ}$. Then $\mathbf{y}^{\circ}+\mathbf{y}=0$ so that $\mathbf{y} \in \mathfrak{s u}_{n}(\mathfrak{O}, \mathfrak{o})$, and $-\mathbf{x}=\mathbf{x}^{\circ}$ implies

$$
\kappa_{0}(\mathbf{x}, \mathbf{y})=\kappa_{0}(\mathbf{x}, \mathbf{z})-\kappa_{0}\left(\mathbf{x}, \mathbf{z}^{\circ}\right)=\kappa_{0}(\mathbf{x}, \mathbf{z})+\kappa_{0}\left(\mathbf{x}^{\circ}, \mathbf{z}^{\circ}\right)=\lambda+\lambda^{\sigma} \not \equiv 0
$$

modulo $\mathfrak{p}=\mathfrak{o} \cap \mathfrak{P}$, as wanted.

7.3. Arithmetic groups of type ${ }^{1} A_{2}$. In this section we prove the assertion of Theorem $\mathbb{C}$ for arithmetic subgroups pertaining to inner forms of $A_{2}$. After a short general setup, covering both inner and outer forms of $A_{2}$, we specialise to inner forms from Section 7.3.1 onward. Outer forms are then treated in Section 7.4 by similar techniques.

Let $k$ be a number field, with ring of integers 0 . Let $S$ be a finite set of places of $k$, containing all the archimedean ones. Let $\mathbf{G}$ be a connected, simply-connected simple, $k$-defined algebraic group of type $A_{2}$. As explained in Appendix $\mathrm{A}$, the classification of semisimple groups over number fields gives a concrete description of the group $\mathbf{G}(k)$ of $k$ rational points in terms of central simple algebras, possibly equipped with an involution. We consider an $S$-arithmetic subgroup $\Gamma$ of $\mathbf{G}(k)$. This means that $\Gamma$ is commensurable to $\mathbf{G}\left(\mathcal{O}_{S}\right):=\mathbf{G}(k) \cap \mathrm{GL}_{N}\left(\mathcal{O}_{S}\right)$, where implicitly one chooses an embedding of $\mathbf{G}$ into $\mathbf{G L}_{N}$ for some $N \in \mathbb{N}$. Suppose that $\Gamma$ has the Congruence Subgroup Property, i.e. that its congruence kernel is finite, so that $\Gamma$ has polynomial representation growth.

We are to show that the abscissa of convergence $\alpha(\Gamma)$ of $\zeta_{\Gamma}(s)$ is equal to 1. Since $\alpha(\Gamma)$ is in fact an invariant of the commensurability class of $\Gamma$, we may - for simplicity suppose that $\Gamma=\mathbf{G}\left(\mathcal{o}_{S}\right)$. By [35, Proposition 4.6], the zeta function $\zeta_{\Gamma}(s)$ thus admits an 'Euler product' decomposition

$$
\zeta_{\Gamma}(s)=\zeta_{\mathbf{G}(\mathbb{C})}(s)^{|k: \mathbb{Q}|} \cdot \prod_{v \notin S} \zeta_{\Gamma_{v}}(s),
$$

where $\Gamma_{v}$ is an open subgroup of $\mathbf{G}\left(\mathcal{O}_{v}\right)$ for every non-archimedean place $v$ and equal to $\mathbf{G}\left(\mathcal{O}_{v}\right)$ for almost all $v$. Note that $\mathbf{G}(\mathbb{C}) \cong \mathrm{SL}_{3}(\mathbb{C})$ as $\mathbf{G}$ is of type $A_{2}$. The abscissa of convergence of each archimedean factor $\zeta_{\mathbf{G}(\mathbb{C})}(s)$ is thus known to be $2 / 3$; see [35. Theorem 5.1]. For any non-archimedean place $v$, there is a finite extension $\mathfrak{O}$ of $\mathfrak{o}=\mathcal{O}_{v}$ such that $\mathbf{G}(\mathfrak{O})$ is commensurable to $\mathrm{SL}_{3}(\mathfrak{O})$; thus Theorem $\mathrm{B}$, in particular (1.3), and Theorem $\mathrm{E}$ show that the abscissa of convergence of the local factor $\zeta_{\Gamma_{v}}(s)$ is at most $2 / 3$. Therefore, in order to prove that the abscissa of convergence $\alpha(\Gamma)$ of the global 
zeta function $\zeta_{\Gamma}(s)$ is equal to 1 , it suffices to specify a suitable finite set $T$ of places of $k$, with $S \subseteq T$, such that the abscissa of convergence of the product $\prod_{v \notin T} \zeta_{\Gamma_{v}}(s)$ is equal to 1.

7.3.1. From now on we specialise to the case where $\mathbf{G}$, and hence $\Gamma$, is of type ${ }^{1} A_{2}$. Let $T$ be the set of all places $v$ of $k$ such that one of the following holds: (i) $v \in S$, (ii) $v$ is dyadic or triadic, i.e. $\mathcal{O}_{v}$ has residue field characteristic 2 or 3 , (iii) $\Gamma_{v}$ is not isomorphic to $\mathrm{SL}_{3}\left(\mathcal{O}_{v}\right)$, (iv) there is a pro- $p$ subgroup $H$ of $\mathrm{SL}_{3}\left(\mathcal{O}_{v}\right)$ containing $\mathrm{SL}_{3}^{1}\left(\mathcal{O}_{v}\right)$ which fails to be saturable or potent. We need to check that $T$ is finite. Clearly, conditions (i) and (ii) only exclude finitely many places. Standard theorems from the theory of orders in central simple algebras over number fields show that (iii) only excludes finitely many valuations. Indeed, $\mathbf{G}(k)$ can be thought of as the norm-1 group of a central simple algebra $A$ over $k$, and $\mathbf{G}\left(\mathcal{O}_{S}\right)$ as the set of norm-1 elements in an $\mathcal{O}_{S}$-order $\Omega$ of $A$. For almost all $v$, the completion $A_{v}$ is split, hence isomorphic to $\operatorname{Mat}_{3}\left(k_{v}\right)$, and the completion $\Omega_{v}$ is a maximal order of $A_{v} \cong \operatorname{Mat}_{3}\left(k_{v}\right)$, hence isomorphic to $\operatorname{Mat}_{3}\left(\mathcal{O}_{v}\right)$; e.g. see [44, Corollary 10.4, Corollary 11.6, Theorem 17.3]. Finally, we turn our attention to the property (iv). For almost all $v$ the field $k_{v}$ has absolute ramification index 1 , and hence [36, III (3.2.7)] shows that the Sylow pro- $p$ subgroup of $\mathrm{SL}_{3}\left(\mathcal{O}_{v}\right)$ is saturable and therefore torsion-free. Since $\operatorname{dim}\left(\mathrm{SL}_{3}\left(\mathcal{O}_{v}\right)\right)$ is uniformly bounded by $8|k: \mathbb{Q}|$, GonzálezSánchez and Klopsch's work [20] on p-adic analytic groups of small dimension shows that the property (iv) can be avoided by excluding finitely many $v$.

We claim that the abscissa of convergence of the product

$$
\varphi(s):=\prod_{v \notin T} \zeta_{\mathrm{SL}_{3}\left(\mathcal{O}_{v}\right)}(s)
$$

is equal to 1. As shown in [3], one can compute via Clifford theory, for each $v \notin T$, an explicit formula for $\zeta_{\mathrm{SL}_{3}\left(\mathcal{O}_{v}\right)}(s)$, starting from our explicit formula (6.5) for the local representation zeta function of the first principal congruence subgroup $\mathrm{SL}_{3}^{1}\left(\mathrm{O}_{v}\right)$. As explained in Remark 7.1, we take a different approach and manufacture, without relying on [3], sufficient approximations $\psi_{v}(s)$ of $\zeta_{\mathrm{SL}_{3}\left(\mathcal{O}_{v}\right)}(s)$ so that

- the abscissa of convergence of $\psi(s):=\prod_{v \notin T} \psi_{v}(s)$ is the same as the one of $\varphi(s)$,

- this abscissa of convergence can be shown to be equal to 1 .

Indeed, for each $v \notin T$ there is a natural decomposition

$$
\zeta_{\mathrm{SL}_{3}\left(\mathcal{O}_{v}\right)}(s)=\left(1+\zeta_{\mathrm{SL}_{3}\left(\mathcal{O}_{v}\right)}^{\mathrm{triv}}(s)\right)+\zeta_{\mathrm{SL}_{3}\left(\mathcal{O}_{v}\right)}^{\mathrm{reg}}(s)+\zeta_{\mathrm{SL}_{3}\left(\mathcal{O}_{v}\right)}^{\mathrm{irreg}}(s)
$$

involving three 'smaller' Dirichlet generating functions. These components enumerate representations which, after restriction to $\mathrm{SL}_{3}^{1}\left(\vartheta_{v}\right)$, involve only the trivial representation, only regular and only irregular representations respectively; recall the terminology introduced in Section 6.1. First we will prove that the abscissa of convergence of the product $\prod_{v \notin T}\left(1+\zeta_{\mathrm{SL}_{3}\left(\mathcal{o}_{v}\right)}^{\text {triv }}(s)\right)$ is equal to 1. Lemma 7.2 and the trivial observation

$$
\begin{aligned}
1+\zeta_{\mathrm{SL}_{3}\left(\mathcal{O}_{v}\right.}^{\mathrm{triv}}(s)+\zeta_{\mathrm{SL}_{3}\left(\mathcal{O}_{v}\right)}^{\mathrm{reg}}(s)+ & \zeta_{\mathrm{SL}_{3}\left(\mathcal{O}_{v}\right)}^{\mathrm{irreg}}(s) \ll \\
& \left(1+\zeta_{\mathrm{SL}_{3}\left(\mathcal{O}_{v}\right)}^{\mathrm{triv}}(s)\right)\left(1+\zeta_{\mathrm{SL}_{3}\left(\mathcal{O}_{v}\right)}^{\mathrm{reg}}(s)+\zeta_{\mathrm{SL}_{3}\left(\mathcal{O}_{v}\right)}^{\mathrm{irreg}}(s)\right)
\end{aligned}
$$

then show that it suffices to prove that the abscissa of convergence of the product $\prod_{v \in \mathcal{V}(k) \backslash T}\left(1+\zeta_{\mathrm{SL}_{3}\left(\mathcal{O}_{v}\right)}^{\mathrm{reg}}(s)+\zeta_{\mathrm{SL}_{3}\left(\mathcal{O}_{v}\right)}^{\mathrm{irreg}}(s)\right)$ is less than or equal to 1 . For this purpose we will approximate the Dirichlet series $\zeta_{\mathrm{SL}_{3}\left(\mathcal{O}_{v}\right)}^{\mathrm{reg}}(s)$ and $\zeta_{\mathrm{SL}_{3}\left(\mathcal{O}_{v}\right)}^{\mathrm{irreg}}(s)$ by suitable rational 
functions $\psi_{v}^{\mathrm{reg}}(s)$ and $\psi_{v}^{\text {irreg }}(s)$ in $q_{v}, q_{v}^{-s}$ and $2^{-s}$, where $q_{v}=\left|\mathcal{O}_{v}: \mathfrak{p}_{v}\right|$ denotes the size of the residue field $\mathcal{o}_{v} / \mathfrak{p}_{v}$ at $v$.

7.3.2. The first factor on the right hand side of (7.6), viz. $1+\zeta_{\mathrm{SL}_{3}\left(\mathrm{O}_{v}\right)}^{\mathrm{triv}}(s)$, arises from representations of $\mathrm{SL}_{3}\left(\mathcal{O}_{v}\right)$ which are trivial on $\mathrm{SL}_{3}^{1}\left(\mathcal{O}_{v}\right)$. Thus $1+\zeta_{\mathrm{SL}_{3}\left(\mathcal{O}_{v}\right)}^{\text {triv }}(s)=\zeta_{\mathrm{SL}_{3}\left(\mathbb{F}_{q_{v}}\right)}(s)$, the zeta function of the finite classical group $\mathrm{SL}_{3}\left(\mathbb{F}_{q_{v}}\right)$.

Proposition 7.9. The abscissa of convergence of the Euler product

$$
\prod_{v \notin T}\left(1+\zeta_{\mathrm{SL}_{3}\left(\mathcal{O}_{v}\right)}^{\mathrm{triv}}(s)\right)=\prod_{v \notin T} \zeta_{\mathrm{SL}_{3}\left(\mathbb{F}_{q_{v}}\right)}(s)
$$

is equal to 1 .

Proof. Let $q$ be a prime power, not divisible by 3. Explicit formulae for the zeta function of the finite classical group $\mathrm{SL}_{3}\left(\mathbb{F}_{q}\right)$ can be computed, for instance, using Deligne-Lusztig Theory; cf. [3] and [40]. One needs to separate the cases $q \equiv_{3} 1$ and $q \equiv_{3} 2$. Indeed, for $q \equiv_{3} 1$ one has

$$
\begin{aligned}
\zeta_{\mathrm{SL}_{3}\left(\mathbb{F}_{q}\right)}(s)=1 & +1 \cdot\left(q^{2}+q\right)^{-s}+(q-2) \cdot\left(q^{2}+q+1\right)^{-s} \\
& +6 \cdot\left((q+1)(q-1)^{2} / 3\right)^{-s}+3 \cdot\left(\left(q^{2}+q+1\right)(q+1) / 3\right)^{-s} \\
& +3^{-1}(q+2)(q-1) \cdot\left((q+1)(q-1)^{2}\right)^{-s}+2^{-1}\left(q^{2}-q\right) \cdot\left(q^{3}-1\right)^{-s} \\
& +1 \cdot q^{-3 s}+(q-2) \cdot\left(q^{3}+q^{2}+q\right)^{-s} \\
& +6^{-1}(q-1)(q-4) \cdot\left(\left(q^{2}+q+1\right)(q+1)\right)^{-s},
\end{aligned}
$$

and for $q \equiv_{3} 2$ one has

$$
\begin{aligned}
\zeta_{\mathrm{SL}_{3}\left(\mathbb{F}_{q}\right)}(s)=1 & +1 \cdot\left(q^{2}+q\right)^{-s}+(q-2) \cdot\left(q^{2}+q+1\right)^{-s} \\
& +3^{-1}\left(q^{2}+q\right) \cdot\left((q+1)(q-1)^{2}\right)^{-s}+2^{-1}\left(q^{2}-q\right) \cdot\left(q^{3}-1\right)^{-s} \\
& +1 \cdot q^{-3 s}+(q-2) \cdot\left(q^{3}+q^{2}+q\right)^{-s} \\
& +6^{-1}(q-2)(q-3) \cdot\left(\left(q^{2}+q+1\right)(q+1)\right)^{-s} .
\end{aligned}
$$

Based on these formulae we can prove our assertion. Indeed, the degree $|k: \mathbb{Q}|$ provides a uniform upper bound for the number of valuations $v \notin T$ which prolong any fixed $p$-adic valuation on $\mathbb{Q}$. Moreover, for each $v$ which prolongs the $p$-adic valuation associated to a prime $p$ we have $p \leq q_{v}=\left|\mathcal{O}_{v}: \mathfrak{p}_{v}\right|$, and the Chebotarev Density Theorem guarantees that for a positive proportion of primes $p$, i.e. for a set of primes $p$ of positive Dirichlet density, there exists a prolongation $v$ such that $p=q_{v}$. In view of the explicit formulae for $\zeta_{\mathrm{SL}_{3}\left(\mathbb{F}_{q}\right)}(s)$ recorded above, this implies that the abscissa of convergence of the product $\prod_{v \notin T} \zeta_{\mathrm{SL}_{3}\left(\mathbb{F}_{q_{v}}\right)}(s)$ is equal to the abscissa of convergence of the sum $\sum_{p}\left(p^{1-2 s}+p^{2-3 s}\right)$, which is equal to 1 .

7.3.3. It remains to bound the abscissa of convergence of the second product on the right hand side of (7.6).

Proposition 7.10. The abscissa of convergence of the Euler product

$$
\prod_{v \notin T}\left(1+\zeta_{\mathrm{SL}_{3}\left(\mathcal{O}_{v}\right)}^{\mathrm{reg}}(s)+\zeta_{\mathrm{SL}_{3}\left(\mathcal{O}_{v}\right)}^{\mathrm{irreg}}(s)\right)
$$

is less than or equal to 1. 
TABLE 7.1. Adjoint orbits in $\mathfrak{s l}_{3}\left(\mathbb{F}_{q}\right)$ under the action of $\mathrm{GL}_{3}\left(\mathbb{F}_{q}\right)$

\begin{tabular}{|l|l|l|l|l|}
\hline type & & number of orbits & size of each orbit & total number \\
\hline \hline 0 & & 1 & 1 & 1 \\
1 & reg. & 1 & $\left(q^{3}-1\right)\left(q^{2}-1\right) q$ & $\approx q^{6}$ \\
2 & irreg. & 1 & $\left(q^{3}-1\right)(q+1)$ & $\approx q^{4}$ \\
3 & irreg. & $q-1$ & $\left(q^{2}+q+1\right) q^{2}$ & $\approx q^{5}$ \\
$4 \mathrm{a}$ & reg. & $(q-1)(q-2) / 6$ & $\left(q^{2}+q+1\right)(q+1) q^{3}$ & $\approx q^{8}$ \\
$4 \mathrm{~b}$ & reg. & $(q-1) q / 2$ & $\left(q^{3}-1\right) q^{3}$ & $\approx q^{8}$ \\
$4 \mathrm{c}$ & reg. & $\left(q^{2}-1\right) / 3$ & $(q+1)(q-1)^{2} q^{3}$ & $\approx q^{8}$ \\
5 & reg. & $(q-1)$ & $\left(q^{3}-1\right)(q+1) q^{2}$ & $\approx q^{7}$ \\
\hline
\end{tabular}

TABLE 7.2. Centralisers in $\mathrm{SL}_{3}\left(\mathbb{F}_{q}\right)$ of elements of $\mathfrak{s l}_{3}\left(\mathbb{F}_{q}\right)$ where $q=p^{r}$

\begin{tabular}{|c|c|c|}
\hline type & & centraliser in $\mathrm{SL}_{3}\left(\mathbb{F}_{q}\right)$ \\
\hline 0 & & $\mathrm{SL}_{3}\left(\mathbb{F}_{q}\right)$ \\
\hline 1 & reg. & $\mu_{3}\left(\mathbb{F}_{q}\right) \times \mathbb{F}_{q}^{+} \times \mathbb{F}_{q}^{+} \cong C_{\operatorname{gcd}(q-1,3)} \times C_{p}^{r} \times C_{p}^{r}$ \\
\hline 2 & irreg. & $\mathbb{F}_{q}^{*} \ltimes \mathcal{H}\left(\mathbb{F}_{q}\right) \cong C_{q-1} \ltimes\left(\left(C_{p}^{r} \times C_{p}^{r}\right) \ltimes C_{p}^{r}\right)$ \\
\hline 3 & irreg. & $\mathrm{GL}_{2}\left(\mathbb{F}_{q}\right)$ \\
\hline $4 \mathrm{a}$ & reg. & $\mathbb{F}_{q}^{*} \times \mathbb{F}_{q}^{*} \cong C_{q-1} \times C_{q-1}$ \\
\hline $4 \mathrm{~b}$ & reg. & $\mathbb{F}_{q^{2}}^{*} \cong C_{q^{2}-1}$ \\
\hline $4 \mathrm{c}$ & reg. & $\operatorname{ker}\left(N_{\mathbb{F}_{q^{3}} \mid \mathbb{F}_{q}}\right) \cong C_{q^{2}+q+1}$ \\
\hline 5 & reg. & $\mathbb{F}_{q}^{*} \times \mathbb{F}_{q}^{+} \cong C_{q-1} \times C_{p}^{r}$ \\
\hline
\end{tabular}

The proof of this proposition will occupy the remainder of the current Section 7.3, By Lemma 7.2, it suffices to find, for all $v \notin T$, approximations $\psi_{v}^{\text {reg }}(s)$ and $\psi_{v}^{\text {irreg }}(s)$ so that, locally,

$$
\zeta_{\mathrm{SL}_{3}\left(\mathcal{O}_{v}\right)}^{\mathrm{reg}}(s) \ll \psi_{v}^{\mathrm{reg}}(s) \quad \text { and } \quad \zeta_{\mathrm{SL}_{3}\left(\mathcal{O}_{v}\right)}^{\mathrm{irreg}}(s) \ll \psi_{v}^{\text {irreg }}(s)
$$

and, globally, the abscissa of convergence of the sum $\sum_{v \notin T}\left(\psi_{v}^{\text {reg }}(s)+\psi_{v}^{\text {irreg }}(s)\right)$ is at most 1.

In order to apply approximative Clifford theory, we require an overview of the elements in $\mathfrak{s l}_{3}\left(\mathbb{F}_{q}\right)$ up to the adjoint action of the group $\mathrm{GL}_{3}\left(\mathbb{F}_{q}\right)$. We distinguish eight different types, labelled $0,1,2,3,4 \mathrm{a}, 4 \mathrm{~b}, 4 \mathrm{c}, 5$. The total number of elements of each type and the isomorphism types of their centralisers in $\mathrm{SL}_{3}\left(\mathbb{F}_{q}\right)$ are summarised in Tables 7.1 and 7.2 see Appendix $\mathbb{B}$ for a short discussion. In Table $7.2, \mu_{3}\left(\mathbb{F}_{q}\right)$ is the group of third roots of unity in $\mathbb{F}_{q}^{*}$, we denote by $\mathbb{F}_{q}^{+}$the additive group of the field $\mathbb{F}_{q}$, we write $\mathcal{H}\left(\mathbb{F}_{q}\right)$ for the Heisenberg group over $\mathbb{F}_{q}$, and $\operatorname{ker}\left(N_{\mathbb{F}_{q^{3}} \mid \mathbb{F}_{q}}\right)$ is the multiplicative group of elements of norm 1 in $\mathbb{F}_{q^{3}} \mid \mathbb{F}_{q}$.

Let $v$ be a place of $k$ with $v \notin T$. The series $\psi_{v}^{\mathrm{reg}}(s)$ is to approximate the second summand in (7.5). It splits into five smaller parts

$$
\psi_{v}^{\mathrm{reg}}(s)=\psi_{v}^{1}(s)+\psi_{v}^{4 \mathrm{a}}(s)+\psi_{v}^{4 \mathrm{~b}}(s)+\psi_{v}^{4 \mathrm{c}}(s)+\psi_{v}^{5}(s)
$$

which correspond - via the diagram (7.4) - to elements of types 1, 4a, 4b, 4c and 5 in the finite Lie algebra $\mathfrak{s l}_{3}\left(\mathbb{F}_{q}\right)$. Each of these summands will be a rational function in $q_{v}, q_{v}^{-s}$ and $2^{-s}$, which we derive using Clifford theory. Corollary 7.6 shows that for elements of types $1,4 \mathrm{a}, 4 \mathrm{~b}, 4 \mathrm{c}$ and 5 the centralisers in $\mathrm{SL}_{3}\left(\mathbb{F}_{q_{v}}\right)$ are isomorphic to the corresponding inertia group quotients. We also remark that types $4 \mathrm{a}, 4 \mathrm{~b}$ and $4 \mathrm{c}$ are somewhat easier to deal with than the rest, since the corresponding inertia group quotients are 'tame'; 
they are cyclic of order coprime to $p$. This implies that characters of these types extend from $\mathrm{SL}_{3}^{1}\left(\mathcal{O}_{v}\right)$ to their respective inertia groups; cf. Lemma 7.4.

The series $\psi_{v}^{\text {irreg }}(s)$ is to approximate the third summand in (7.5). It splits into two smaller parts

$$
\psi_{v}^{\text {irreg }}(s)=\psi_{v}^{2}(s)+\psi_{v}^{3}(s)
$$

which correspond - via the diagram (7.4) - to elements of types 2 and 3 in $\mathfrak{s l}_{3}\left(\mathbb{F}_{q_{v}}\right)$. As before, each of these summands will be a rational function in $q_{v}, q_{v}^{-s}$ and $2^{-s}$, obtained by means of Clifford theory. Corollary 7.6 does not apply to elements of types 2 and 3 and the inertia group quotients are, in fact, frequently smaller than the recorded centralisers in $\mathrm{SL}_{3}\left(\mathbb{F}_{q_{v}}\right)$.

For every prime $p$ there are at most $|k: \mathbb{Q}|$ prolongations $v$ to $k$ of the $p$-adic valuation on $\mathbb{Q}$. Moreover, for any prolongation $v$ of the $p$-adic valuation associated to a prime $p$, the size $q_{v}$ of the corresponding residue field satisfies $p \leq q_{v} \leq p^{|k: \mathbb{Q}|}$. Therefore, in order to show that the abscissa of convergence of the $\operatorname{sum} \sum_{v \notin T}\left(\psi_{v}^{\mathrm{reg}}(s)+\psi_{v}^{\text {irreg }}(s)\right)$ is less than or equal to 1, it will be enough to show that for each of the rational functions $f\left(q, q^{-s}, 2^{-s}\right)$ in $q, q^{-s}$ and $2^{-s}$ which we derive below to bound the contributions from representations of the various types and for each fixed exponent $t$ in the range $1 \leq t \leq$ $|k: \mathbb{Q}|$, the individual series $\sum_{q} f\left(q, q^{-s}, 2^{-s}\right)$ converges for $\operatorname{Re}(s)>1$, where $q$ runs over all prime powers $p^{t}$ with exponent $t$. In practice, it will be enough to consider the 'worst' case $t=1$, i.e. $q$ running over all primes.

7.3.4. We now work out the details case by case. Let $v$ be a place of $k$ with $v \notin T$. In considering the local situation at $v$, it is convenient to adopt a simplified notation which suppresses explicit references to $v$. We write $\mathfrak{o}:=\mathcal{O}_{v}, \mathfrak{p}:=\mathfrak{p}_{v}$ for the maximal ideal and $\mathbb{F}_{q}:=\mathfrak{o} / \mathfrak{p}$ for the residue field, where $q:=q_{v}$. Furthermore, we write $\psi_{\mathfrak{o}}^{\text {reg }}(s):=\psi_{v}^{\text {reg }}(s)$, $\psi_{\mathfrak{o}}^{\text {irreg }}(s):=\psi_{v}^{\text {irreg }}(s), \psi_{\mathfrak{o}}^{1}(s):=\psi_{v}^{1}(s)$, etc.

Set $G:=\mathrm{SL}_{3}(\mathfrak{o}), N:=\mathrm{SL}_{3}^{1}(\mathfrak{o})$ and $\gamma(q):=q^{-8}|G: N|=\left(1-q^{-3}\right)\left(1-q^{-2}\right)$. We use the generous bound $\gamma(q)>2^{-1}$. In each case we need to multiply the approximate contribution number from the last column of Table [7.1, an approximation of the translation factor $\left|G: I_{G}(\vartheta)\right|^{-1-s} \zeta_{G, \vartheta}(s)$, in accordance with (7.2), and (an approximation of) the appropriate series factor, appearing on one of the right hand sides of (6.6). In the case of regular representations we use the precise translation factor $\left(1-q^{2-3 s}\right)^{-1}$; for irregular representations we use the generous estimate

$$
\frac{1-q^{1-2 s}-q^{2-3 s}+q^{4-2 s}}{\left(1-q^{1-2 s}\right)\left(1-q^{2-3 s}\right)} \ll \frac{1+q^{4-2 s}}{\left(1-q^{1-2 s}\right)\left(1-q^{2-3 s}\right)} .
$$

Lemma 7.4 plays a key role in deriving a suitable estimate of the term $\zeta_{G, \vartheta}(s)$. We deal with the different cases arising from Table 7.1 in the following order: 4a, 4b, 4c, 5, 2, 1, 3 .

Type 4a. According to Table 7.1 the number of elements of type 4a is bounded by $q^{8}$. Let $\vartheta \in \operatorname{Irr}(N)$ be one of the representations of type 4a. Then, according to Table 7.2 and Corollary 17.6, the inertia quotient is $I_{G}(\vartheta) / N \cong C_{q-1} \times C_{q-1}$. Hence, by Lemma 7.4 (1), we can approximate $\zeta_{G, \vartheta}=\zeta_{I_{G}(\vartheta) / N}(s)=(q-1)^{2}$ by $q^{2}$ and bound $\left|G: I_{G}(\vartheta)\right|$ from below by $\gamma(q) q^{6}>2^{-1} q^{6}$. In view of Remark 7.3 , this gives the approximate translation factor $q^{2} \cdot\left(2^{-1} q^{6}\right)^{-1-s}=2^{1+s} q^{-4-6 s}$. The series factor is $\left(1-q^{2-3 s}\right)^{-1}$. Altogether elements of type 4 a contribute to $\psi_{\mathfrak{o}}^{\text {reg }}(s)$ with a summand

$$
\psi_{\mathfrak{o}}^{4 \mathrm{a}}(s)=q^{8} \cdot 2^{1+s} q^{-4-6 s} \cdot\left(1-q^{2-3 s}\right)^{-1}=2^{1+s} q^{4-6 s}\left(1-q^{2-3 s}\right)^{-1} .
$$


The sum $\sum_{q} 2^{1+s} q^{4-6 s}\left(1-q^{2-3 s}\right)^{-1}$, where $q$ runs over all primes, converges for $s \in \mathbb{C}$ with $\operatorname{Re}(s)>5 / 6$, and hence for $s \in \mathbb{C}$ with $\operatorname{Re}(s)>1$, as wanted.

Types $4 \mathrm{~b}$ and $4 \mathrm{c}$. The argument is very similar to the one for type $4 \mathrm{a}$.

Type 5. According to Table 7.1 the number of elements of type 5 is bounded by $q^{7}$. Let $\vartheta \in \operatorname{Irr}(N)$ be one of the representations of type 5 . Then according to Table 7.2 and Corollary 7.6 the inertia group quotient is $I_{G}(\vartheta) / N \cong C_{q-1} \times C_{p}^{r}$. Let $P / N$ denote the Sylow- $p$ subgroup of $I_{G}(\vartheta) / N$, contained in a Sylow- $p$ subgroup $H / N$ of $G / N$, and recall that our definition of $T$ guarantees that the pro- $p$ groups $N, P=I_{H}(\vartheta)$ and $H$ are saturable and potent. By Lemma 7.8, the saturable Lie lattice corresponding to $P$ is an $\mathfrak{o}$-Lie lattice. Thus, by Corollary [3.2, the character degrees of $P$ and $N$ are powers of $q$. By Lemma 7.4. $\vartheta$ extends to $P$ so that $\zeta_{G, \vartheta}(s)=(q-1) q \ll q^{2}$. We bound $\left|G: I_{G}(\vartheta)\right|$ from below by $\gamma(q) q^{6}>2^{-1} q^{6}$. In view of Remark 7.3 , this gives the approximate translation factor $q^{2} \cdot\left(2^{-1} q^{6}\right)^{-1-s}=2^{1+s} q^{-4-6 s}$. The series factor is $\left(1-q^{2-3 s}\right)^{-1}$. Altogether elements of type 5 contribute to $\psi_{\mathfrak{o}}^{\text {reg }}(s)$ with a summand

$$
\psi_{\mathfrak{o}}^{5}(s)=q^{7} \cdot 2^{1+s} q^{-4-6 s} \cdot\left(1-q^{2-3 s}\right)^{-1}=2^{1+s} q^{3-6 s}\left(1-q^{2-3 s}\right)^{-1} .
$$

The sum $\sum_{q} 2^{1+s} q^{3-6 s}\left(1-q^{2-3 s}\right)^{-1}$, where $q$ runs over all primes, converges for $s \in \mathbb{C}$ with $\operatorname{Re}(s)>4 / 6=2 / 3$, and hence for $s \in \mathbb{C}$ with $\operatorname{Re}(s)>1$, as wanted.

Type 2. According to Table 7.1 there are less than $2 q^{4}$ contributing elements of type 2 . Let $\vartheta \in \operatorname{Irr}(N)$ be one of the representations of type 2 . Without determining the precise inertia group quotient, it suffices for our estimate to know that $I_{G}(\vartheta) / N$ is a subgroup of the centraliser group modulo $\mathfrak{p}$, as described in Table 7.2. This centraliser is an extension of the Heisenberg group $\mathcal{H}\left(\mathbb{F}_{q}\right)$ over $\mathbb{F}_{q}$ by the multiplicative group $\mathbb{F}_{q}^{*} \cong C_{q-1}$, where the top group acts with a kernel of $\operatorname{size} \operatorname{gcd}(q-1,3)$ and with orbits of equal length $(q-1) / \operatorname{gcd}(q-1,3)$ on the non-trivial elements of $\mathcal{H}\left(\mathbb{F}_{q}\right) /\left[\mathcal{H}\left(\mathbb{F}_{q}\right), \mathcal{H}\left(\mathbb{F}_{q}\right)\right]$; see $(\underline{B} .2)$. In order to make the argument independent of whether $\vartheta$ extends to $I_{G}(\vartheta)$ or not, we include both possibilities in our approximation.

First suppose that $\vartheta$ extends to $I_{G}(\vartheta)$. Then we compute the approximation as follows. If the inertia group quotient contains the full Heisenberg group $\mathcal{H}\left(\mathbb{F}_{q}\right)$, then it is isomorphic to an extension of the Heisenberg group by a cyclic group of order $m \mid(q-1)$. Put $m_{0}:=\operatorname{gcd}(m, 3)$ and $m_{1}:=m / m_{0}$. The zeta function of the Heisenberg group is known to be $\zeta_{\mathcal{H}\left(\mathbb{F}_{q}\right)}(s)=q^{2}+(q-1) q^{-s}$; see, for instance, [30]. A straightforward application of Clifford theory, in the spirit of (7.2), thus yields the following estimate for the zeta function of the inertia group quotient:

$$
\zeta_{I_{G}(\vartheta) / N}(s) \ll m+m_{0} m_{1}^{-1-s}\left(q^{2}-1\right)+m(q-1) q^{-s} .
$$

We bound the index $\left|G: I_{G}(\vartheta)\right|$ from below by $\gamma(q) q^{5} m^{-1}>2^{-1} q^{5} m^{-1}$. Noting that

$$
\begin{aligned}
(m & \left.+m_{0} m_{1}^{-1-s}\left(q^{2}-1\right)+m(q-1) q^{-s}\right) \cdot 2^{1+s} q^{-5-5 s} m^{1+s} \\
& \ll 2^{1+s} q^{-5-5 s}\left(m^{2+s}+m_{0}^{2+s} q^{2}+m^{2+s} q^{1-s}\right) \\
& \ll 2^{1+s}\left(q^{-3-4 s}+9 q^{-3-4 s}+q^{-2-5 s}\right) \\
& =2^{1+s}\left(10 q^{-3-4 s}+q^{-2-5 s}\right)
\end{aligned}
$$

and approximating the required series factor by $\left(1+q^{4-2 s}\right)\left(1-q^{1-2 s}\right)^{-1}\left(1-q^{2-3 s}\right)^{-1}$, we obtain the contributing summand

$$
\begin{aligned}
\psi_{\mathfrak{o}}^{2(\mathrm{a})}(s) & =2 q^{4} \cdot 2^{1+s}\left(10 q^{-3-4 s}+q^{-2-5 s}\right) \cdot\left(1+q^{4-2 s}\right)\left(1-q^{1-2 s}\right)^{-1}\left(1-q^{2-3 s}\right)^{-1} \\
& =2^{2+s}\left(10 q^{1-4 s}+10 q^{5-6 s}+q^{2-5 s}+q^{6-7 s}\right)\left(1-q^{1-2 s}\right)^{-1}\left(1-q^{2-3 s}\right)^{-1} .
\end{aligned}
$$


The sums $\sum_{q} 2^{2+s} q^{a-b s}\left(1-q^{1-2 s}\right)^{-1}\left(1-q^{2-3 s}\right)^{-1},(a, b) \in\{(1,4),(5,6),(2,5),(6,7)\}$, where $q$ runs over all primes, converge for $s \in \mathbb{C}$ with $\operatorname{Re}(s)>1$, as wanted.

Now suppose that the inertia group quotient does not contain the full Heisenberg group $\mathcal{H}\left(\mathbb{F}_{q}\right)$. From Lemma 7.8 we conclude that $\left|I_{G}(\vartheta): N\right| \leq q^{3}$ and we approximate $\zeta_{I_{G}(\vartheta) / N}(s)$ generously by $q^{3}$. Bounding $\left|G: I_{G}(\vartheta)\right|$ from below by $\gamma(q) q^{5}>2^{-1} q^{5}$ and approximating the required series factor as before, we obtain the contributing summand

$$
\begin{aligned}
\psi_{\mathfrak{o}}^{2(\mathrm{~b})}(s) & =2 q^{4} \cdot q^{3} \cdot 2^{1+s} q^{-5-5 s} \cdot\left(1+q^{4-2 s}\right)\left(1-q^{1-2 s}\right)^{-1}\left(1-q^{2-3 s}\right)^{-1} \\
& =2^{2+s}\left(q^{2-5 s}+q^{6-7 s}\right)\left(1-q^{1-2 s}\right)^{-1}\left(1-q^{2-3 s}\right)^{-1} .
\end{aligned}
$$

The sums $\sum_{q} 2^{2+s} q^{a-b s}\left(1-q^{1-2 s}\right)^{-1}\left(1-q^{2-3 s}\right)^{-1},(a, b) \in\{(2,5),(6,7)\}$, where $q$ runs over all primes, converges for $s \in \mathbb{C}$ with $\operatorname{Re}(s)>1$, as wanted.

Next suppose that $\vartheta$ does not extend to $I_{G}(\vartheta)$. We use Lemma 7.4)(2), together with the estimate $q^{4}$ for $\left|I_{G}(\vartheta): N\right|$, the lower bound $\gamma(q) q^{4}>2^{-1} q^{4}$ for $\left|G: I_{G}(\vartheta)\right|$ and the same approximation of the required series factor as before, to obtain the approximation

$$
\begin{aligned}
\psi_{\mathfrak{o}}^{2(\mathrm{c})}(s) & =2 q^{4} \cdot q^{4} \cdot q^{-2-s} \cdot 2^{1+s} q^{-4-4 s} \cdot\left(1+q^{4-2 s}\right)\left(1-q^{1-2 s}\right)^{-1}\left(1-q^{2-3 s}\right)^{-1} \\
& =2^{2+s}\left(q^{2-5 s}+q^{6-7 s}\right)\left(1-q^{1-2 s}\right)^{-1}\left(1-q^{2-3 s}\right)^{-1} .
\end{aligned}
$$

The sums $\sum_{q} 2^{2+s} q^{a-b s}\left(1-q^{1-2 s}\right)^{-1}\left(1-q^{2-3 s}\right)^{-1},(a, b) \in\{(2,5),(6,7)\}$, where $q$ runs over all primes, converge for $s \in \mathbb{C}$ with $\operatorname{Re}(s)>1$, as wanted.

Type 1. According to Table 7.1, the number of elements of type 1 is bounded by $q^{6}$. Let $\vartheta \in \operatorname{Irr}(N)$ be one of the representations of type 1 .

First suppose that $\vartheta$ extends to $I_{G}(\vartheta)$. Then, according to Table 7.2 and Corollary 7.6 . the inertia group quotient is $I_{G}(\vartheta) / N \cong C_{\operatorname{gcd}(q-1,3)} \times C_{p}^{r} \times C_{p}^{r}$. Hence we can approximate $\zeta_{I_{G}(\vartheta) / N}(s)=\operatorname{gcd}(q-1,3) q^{2}$ by $2^{2} q^{2}$ and bound $\left|G: I_{G}(\vartheta)\right|$ from below by $\gamma(q) q^{6}>$ $2^{-3} q^{6}$. This gives the approximate translation factor $2^{2} q^{2} \cdot\left(2^{-3} q^{6}\right)^{-1-s}=2^{5+3 s} q^{-4-6 s}$. The series factor is $\left(1-q^{2-3 s}\right)^{-1}$. Altogether this gives a contributing summand

$$
\psi_{\mathfrak{o}}^{1(\mathrm{a})}(s)=q^{6} \cdot 2^{5+3 s} q^{-4-6 s} \cdot\left(1-q^{2-3 s}\right)^{-1}=2^{5+3 s} q^{2-6 s}\left(1-q^{2-3 s}\right)^{-1} .
$$

The sum $\sum_{q} 2^{5+3 s} q^{2-6 s}\left(1-q^{2-3 s}\right)^{-1}$, where $q$ runs over all primes, converges for $s \in \mathbb{C}$ with $\operatorname{Re}(s)>2 / 3$, hence for $s \in \mathbb{C}$ with $\operatorname{Re}(s)>1$, as wanted.

Next suppose that $\vartheta$ does not extend to $I_{G}(\vartheta)$. Let $P / N$ denote the Sylow- $p$ subgroup of $I_{G}(\vartheta) / N$, contained in a Sylow- $p$ subgroup $H / N$ of $G / N$, and recall that our definition of $T$ guarantees that the pro- $p$ groups $N, P=I_{H}(\vartheta)$ and $H$ are saturable and potent. By Lemma 7.8, the saturable Lie lattice corresponding to $P$ is an $\mathfrak{o}$-Lie lattice. Thus, by Corollary 3.2 , the character degrees of $P$ and $N$ are powers of $q$. Therefore, according to Lemma 7.4 (2), we can use similar estimates as above to approximate the contribution by

$$
\psi_{\mathfrak{o}}^{1(\mathrm{~b})}(s)=q^{6} \cdot 2^{2} q^{2} \cdot q^{-2-s} \cdot 2^{3+3 s} q^{-6-6 s} \cdot\left(1-q^{2-3 s}\right)^{-1}=2^{5+3 s} q^{-7 s}\left(1-q^{2-3 s}\right)^{-1} .
$$

The sum $\sum_{q} 2^{5+3 s} q^{-7 s}\left(1-q^{2-3 s}\right)^{-1}$, where $q$ runs over all primes, converges for $s \in \mathbb{C}$ with $\operatorname{Re}(s)>2 / 3$, hence for $s \in \mathbb{C}$ with $\operatorname{Re}(s)>1$, as wanted.

Type 3. According to Table 7.1, the number of elements of type 3 is bounded by $q^{5}$. The difficulty in this case is that the precise inertia group quotient is typically significantly smaller than $\mathrm{GL}_{2}\left(\mathbb{F}_{q}\right)$, the supergroup given in Table 7.2. Recall that $G=\mathrm{SL}_{3}(\mathfrak{o})$ and write $\mathfrak{g}:=\mathfrak{s l}_{3}(\mathfrak{o})$. There is no direct Lie correspondence between the compact $p$-adic analytic group $G$ and the $\mathfrak{o}$-Lie lattice $\mathfrak{g}$, but we will use an approximate correspondence. An irreducible character $\vartheta$ of $N=\mathrm{SL}_{3}^{1}(\mathfrak{o})$ is represented by a Kirillov orbit $\Omega=\omega^{N}$ in $\widehat{\mathfrak{n}}$, where $\mathfrak{n}:=\mathfrak{s l}_{3}^{1}(\mathfrak{o})=\mathfrak{p g}$. By means of the normalised Killing form, the inertia group 
$I_{G}(\vartheta)$ can be described in terms of the centraliser $\mathrm{C}_{G}\left(x+\mathfrak{p}^{n} \mathfrak{n}\right)$, formed with respect to the adjoint action of $G$, for a suitable $x \in \mathfrak{n}^{*}$ and level $n \in \mathbb{N}$. The groups and Lie lattices involved, i.e. $G, \mathfrak{g}$, their respective subobjects and the relevant actions, naturally embed into the $\mathfrak{o}$-order $A:=\operatorname{Mat}_{3}(\mathfrak{o})$. We are to determine

$$
I_{G}(\vartheta) / N=\mathrm{C}_{G}\left(x+\mathfrak{p}^{n} \mathfrak{n}\right) N / N,
$$

equivalently its image $H$ under the natural inclusion into the $\mathbb{F}_{q}$-vector space

$$
V:=\left(\mathrm{C}_{A}\left(x+\mathfrak{p}^{n} \mathfrak{n}\right)+\mathfrak{p} A\right) / \mathfrak{p} A .
$$

As we are interested in the case where $\vartheta$ is of type 3 , we may assume without loss of generality that

$$
x+\mathfrak{p n}=\left(\begin{array}{ccc}
\lambda & & \\
& \lambda & \\
& & -2 \lambda
\end{array}\right)+\mathfrak{p n} \quad \text { where } \lambda \in \mathfrak{p} \backslash \mathfrak{p}^{2}
$$

cf. (B.3). In this situation $V$ is a subspace of the 5 -dimensional $\mathbb{F}_{q}$-vector space

$$
\left\{\left(\begin{array}{cc}
B & \\
& a
\end{array}\right) \mid B \in \operatorname{Mat}_{2}\left(\mathbb{F}_{q}\right) \text { and } a \in \mathbb{F}_{q}\right\} \text {. }
$$

Recalling that the characteristic of the residue field $\mathbb{F}_{q}$ is not 3 , we observe that

- the $\mathbb{F}_{q^{-}}$subspace $V_{0}:=\left(\mathrm{C}_{\mathfrak{g}}\left(x+\mathfrak{p}^{n} \mathfrak{n}\right)+\mathfrak{p} A\right) / \mathfrak{p} A$ of $V$, consisting of cosets of matrices of trace 0 , has co-dimension 1 ;

- the relevant subset $H$ of $V$, consisting of cosets of matrices of determinant 1 , has index at least $(q-1) / \operatorname{gcd}(q-1,3) \geq\lfloor(q-1) / 3\rfloor$ in the group formed by all cosets of invertible matrices in $V$.

First suppose that $V$ has $\mathbb{F}_{q}$-dimension at least 4 . Then $\operatorname{dim}_{\mathbb{F}_{q}}\left(V_{0}\right) \geq 3$ and, considering the image of $V_{0} \cong\left(\mathrm{C}_{\mathfrak{g}}\left(x+\mathfrak{p}^{n} \mathfrak{n}\right)+\mathfrak{p g}\right) / \mathfrak{p g}$ under multiplication by the uniformiser $\pi$ in $\left(\mathrm{C}_{\mathfrak{n}}\left(x+\mathfrak{p}^{n} \mathfrak{n}\right)+\mathfrak{p} \mathfrak{n}\right) / \mathfrak{p n}$, we conclude that $\mathrm{C}_{\mathfrak{n}}\left(x+\mathfrak{p}^{n} \mathfrak{n}\right)$, regarded as an $\mathfrak{o}$-submodule of $\mathfrak{n}$, has elementary divisors $\left(\mathfrak{o}, \mathfrak{o}, \mathfrak{o}, \mathfrak{o}, \mathfrak{p}^{n}, \mathfrak{p}^{n}, \mathfrak{p}^{n}, \mathfrak{p}^{n}\right)$. Thus $\left|\mathfrak{n}: \mathrm{C}_{\mathfrak{n}}\left(x+\mathfrak{p}^{n} \mathfrak{n}\right)\right|=q^{4 n}$.

Our analysis in Section 6.1 shows that $x+\mathfrak{p}^{n} \mathfrak{n}$ corresponds to a point modulo $\mathfrak{p}^{n}$ on the variety $\mathcal{W}_{1}$ defined by the set $F_{3}(\mathbf{Y})$ of polynomials which controls the integrand of (6.2). These are rare; if we fix $\lambda$ in (7.7) there are $q^{5 n}$ lifts modulo $\mathfrak{p}^{n}$. The resulting 'series factor' is $\sum_{n=0}^{\infty} q^{5 n} q^{-4 n(s+2) / 2}=1 /\left(1-q^{1-2 s}\right)$, and the inertia group quotient $I_{G}(\vartheta) / N$ is isomorphic to $\mathrm{GL}_{2}\left(\mathbb{F}_{q}\right)$ so that

$$
\left|G: I_{G}(\vartheta)\right|=\left|\mathrm{SL}_{3}\left(\mathbb{F}_{q}\right)\right|\left|\mathrm{GL}_{2}\left(\mathbb{F}_{q}\right)\right|^{-1}=\left(q^{2}+q+1\right) q^{2} \geq q^{4} .
$$

Let $P / N$ denote the Sylow- $p$ subgroup of $I_{G}(\vartheta) / N$, contained in a Sylow- $p$ subgroup $H / N$ of $G / N$. The group $P / N$ is isomorphic to the additive group $\mathbb{F}_{q}^{+}$. Recall that our definition of $T$ guarantees that the pro- $p$ groups $N, P=I_{H}(\vartheta)$ and $H$ are saturable and potent. By Lemma 7.8, the saturable Lie lattice corresponding to $P$ is an $\mathfrak{o}$-Lie lattice. Thus, by Corollary [3.2, the character degrees of $P$ and $N$ are powers of $q$. By Lemma 7.4, the character $\vartheta$ extends to $P$ so that

$$
\begin{aligned}
\zeta_{G, \vartheta}(s) & =\zeta_{\mathrm{GL}_{2}\left(\mathbb{F}_{q}\right)}(s) \\
& =(q-1)\left(1+q^{-s}+(q-2) / 2 \cdot(q+1)^{-s}+q / 2 \cdot(q-1)^{-s}\right) \\
& \ll q+2^{s} q^{2-s} .
\end{aligned}
$$


Thus the contributing summand is

$$
\begin{aligned}
\psi_{\mathfrak{o}}^{3(\mathrm{a})}(s) & =q^{5} \cdot\left(q+2^{s} q^{2-s}\right) \cdot q^{-4-4 s} \cdot\left(1-q^{1-2 s}\right)^{-1} \\
& =\left(q^{2-4 s}+2^{s} q^{3-5 s}\right)\left(1-q^{1-2 s}\right)^{-1} .
\end{aligned}
$$

The sums $\sum_{q} q^{2-4 s}\left(1-q^{1-2 s}\right)^{-1}$ and $\sum_{q} 2^{s} q^{3-5 s}\left(1-q^{1-2 s}\right)^{-1}$, where $q$ runs over all primes, converge for $s \in \mathbb{C}$ with $\operatorname{Re}(s)>4 / 5$, hence for $s \in \mathbb{C}$ with $\operatorname{Re}(s)>1$ as wanted.

Now suppose that the dimension of $V$ is at most 3 . Then

$$
\left|I_{G}(\vartheta): N\right|=|H| \leq\left(q^{3}-1\right)\lfloor(q-1) / 3\rfloor^{-1} \leq 6 q^{2},
$$

and we approximate $\zeta_{I_{G}(\vartheta) / N}(s)$ generously by $2^{3} q^{2}$. Bounding $\left|G: I_{G}(\vartheta)\right|$ from below by $\gamma(q) 2^{-3} q^{6}>2^{-4} q^{6}$ and approximating the required series factor by $\left(1+q^{4-2 s}\right)(1-$ $\left.q^{1-2 s}\right)^{-1}\left(1-q^{2-3 s}\right)^{-1}$, we obtain the contribution

$$
\begin{aligned}
\psi_{\mathfrak{o}}^{3(\mathrm{~b})}(s) & =q^{5} \cdot 2^{3} q^{2} \cdot 2^{4+4 s} q^{-6-6 s} \cdot\left(1+q^{4-2 s}\right)\left(1-q^{1-2 s}\right)^{-1}\left(1-q^{2-3 s}\right)^{-1} \\
& =2^{7+4 s}\left(q^{1-6 s}+q^{5-8 s}\right)\left(1-q^{1-2 s}\right)^{-1}\left(1-q^{2-3 s}\right)^{-1} .
\end{aligned}
$$

The sums $\sum_{q} 2^{7+4 s} q^{a-b s}\left(1-q^{1-2 s}\right)^{-1}\left(1-q^{2-3 s}\right)^{-1},(a, b) \in\{(1,6),(5,8)\}$, where $q$ runs over all primes, converge for $s \in \mathbb{C}$ with $\operatorname{Re}(s)>3 / 4$, hence for $s \in \mathbb{C}$ with $\operatorname{Re}(s)>1$ as wanted.

This finishes the proof of Proposition 17.10, Together with Proposition 7.9 and the reductions leading up to these two propositions, this also completes the proof of Theorem $\mathbb{C}$ in the case of inner forms.

7.4. Arithmetic groups of type ${ }^{2} A_{2}$. In this section we prove the assertion of Theorem $\mathbb{C}$ for arithmetic subgroups pertaining to outer forms of $A_{2}$. We work in the same setup as described at the beginning of Section 7.3. except that we now assume that the $S$-arithmetic group $\Gamma=\mathbf{G}\left(\mathcal{O}_{S}\right)$ is an outer form, i.e. of type ${ }^{2} A_{2}$. Again, it suffices to specify a suitable finite set $T$ of places of $k$, with $S \subseteq T$, such that the abscissa of convergence of the product $\prod_{v \notin T} \zeta_{\Gamma_{v}}(s)$ is equal to 1 . The argument is going to be very similar to the one given for inner forms.

7.4.1. In this section, it is convenient to write $\mathrm{SU}_{3}\left(\mathcal{O}_{v}\right)$ for the group $\mathrm{SU}_{3}\left(\mathfrak{O}, \mathcal{O}_{v}\right)$, where $\mathfrak{O}$ is an unramified quadratic extension of $\mathcal{O}_{v}$. Similarly, we write $\mathrm{SU}_{3}\left(\mathbb{F}_{q}\right)$ for the finite group $\mathrm{SU}_{3}\left(\mathbb{F}_{q^{2}}, \mathbb{F}_{q}\right)$ and $\mathfrak{s u}_{3}\left(\mathbb{F}_{q}\right)$ for the finite Lie algebra $\mathfrak{s u}_{3}\left(\mathbb{F}_{q^{2}}, \mathbb{F}_{q}\right)$ over a residue field $\mathbb{F}_{q}$, etc. Let $T$ be the set of all places $v$ of $k$ such that one of the following holds: (i) $v \in S$, (ii) $v$ is dyadic or triadic, i.e. $\mathcal{O}_{v}$ has residue field characteristic 2 or 3 , (iii) $\Gamma_{v}$ is not isomorphic to $\mathrm{SL}_{3}\left(\mathcal{O}_{v}\right)$ or $\mathrm{SU}_{3}\left(\mathcal{O}_{v}\right)$, (iv) there is a pro-p subgroup $H$ of $\mathrm{SL}_{3}\left(\mathcal{O}_{v}\right)$ containing $\mathrm{SL}_{3}^{1}\left(\mathcal{O}_{v}\right)$, or of $\mathrm{SU}_{3}\left(\mathcal{O}_{v}\right)$ containing $\mathrm{SU}_{3}^{1}\left(\mathcal{O}_{v}\right)$, which fails to be saturable or potent. Then, similarly as in Section 7.3.1, one checks readily that $T$ is finite. In particular, the discussion in Appendix $\mathrm{A}$ shows that (iii) singles out only finitely many places and, furthermore, that $V_{\mathrm{SL}}:=\left\{v \notin T \mid \Gamma_{v} \cong \mathrm{SL}_{3}\left(\mathcal{O}_{v}\right)\right\}$ and $V_{\mathrm{SU}}:=\left\{v \notin T \mid \Gamma_{v} \cong \mathrm{SU}_{3}\left(\mathcal{O}_{v}\right)\right\}$ have positive Dirichlet density within the set of all places of $k$.

We claim that the abscissa of convergence of the product

$$
\prod_{v \notin T} \zeta_{\Gamma_{v}}(s)=\prod_{v \in V_{\mathrm{SL}}} \zeta_{\mathrm{SL}_{3}\left(\mathcal{O}_{v}\right)}(s) \prod_{v \in V_{\mathrm{SU}}} \zeta_{\mathrm{SU}_{3}\left(\mathcal{O}_{v}\right)}(s)
$$

is equal to 1. Again we take an approximative approach. The first product on the right hand side has abscissa of convergence equal to 1 by the arguments given in Section 7.3 . (For this we make use of the fact that for a positive proportion of primes $p$ there exists a prolongation $v \in V_{\mathrm{SL}}$ such that $p=q_{v}$. This follows from the Chebotarev Density 
Theorem, applied to the quadratic extension $K$ of $k$ which appears in the description of $\mathbf{G}(k)$ in Appendix $\mathrm{A}$ ) It suffices to show that the abscissa of convergence of the product

$$
\varphi(s):=\prod_{v \in V_{\mathrm{SU}}} \zeta_{\mathrm{SU}_{3}\left(\mathcal{O}_{v}\right)}(s)
$$

is less than or equal to 1 .

Similarly to (7.5), for each $v \in V_{\mathrm{SU}}$ there is a natural decomposition

$$
\zeta_{\mathrm{SU}_{3}\left(\mathcal{O}_{v}\right)}(s)=\left(1+\zeta_{\mathrm{SU}_{3}\left(\mathcal{O}_{v}\right)}^{\mathrm{triv}}(s)\right)+\zeta_{\mathrm{SU}_{3}\left(\mathcal{O}_{v}\right)}^{\mathrm{reg}}(s)+\zeta_{\mathrm{SU}_{3}\left(\mathcal{O}_{v}\right)}^{\mathrm{irreg}}(s)
$$

involving three 'smaller' Dirichlet generating functions. These components enumerate representations which, after restriction to $\mathrm{SU}_{3}^{1}\left(\mathcal{O}_{v}\right)$, involve only the trivial, only regular and only irregular representations respectively; recall the terminology introduced in Section 6.1. Similarly to Sections 7.3.3 and 7.3.4, we will approximate the Dirichlet series $\zeta_{\mathrm{SU}_{3}\left(\mathcal{O}_{v}\right)}^{\mathrm{reg}}(s)$ and $\zeta_{\mathrm{SU}_{3}\left(\mathcal{O}_{v}\right)}^{\mathrm{irreg}}(s)$ by suitable rational functions $\psi_{v}^{\mathrm{reg}}(s)$ and $\psi_{v}^{\text {irreg }}(s)$ in $q_{v}, q_{v}^{-s}$ and $2^{-s}$, where $q_{v}=\left|\mathcal{O}_{v}: \mathfrak{p}_{v}\right|$ denotes the size of the residue field $\mathcal{O}_{v} / \mathfrak{p}_{v}$ at $v$. Based on these approximations, an argument akin to the one given in Section 7.3 shows that the abscissa of convergence of $\varphi(s)$ is less than or equal to 1 . This in turn will yield a proof of Theorem $\mathrm{C}$ for outer forms.

7.4.2. The first summand in (7.9) , viz. $1+\zeta_{\mathrm{SU}_{3}\left(\mathcal{O}_{v}\right)}^{\text {triv }}(s)$, arises from representations of $\mathrm{SU}_{3}\left(\mathcal{O}_{v}\right)$ which are trivial on $\mathrm{SU}_{3}^{1}\left(\mathcal{O}_{v}\right)$. Thus $1+\zeta_{\mathrm{SU}_{3}\left(\mathcal{O}_{v}\right)}^{\mathrm{triv}}(s)=\zeta_{\mathrm{SU}_{3}\left(\mathbb{F}_{q v}\right)}(s)$, the zeta function of the finite classical group $\mathrm{SU}_{3}\left(\mathbb{F}_{q_{v}}\right)$.

Proposition 7.11. The abscissa of convergence of the Euler product

$$
\prod_{v \in V_{\mathrm{SU}}}\left(1+\zeta_{\mathrm{SU}_{3}\left(\mathcal{O}_{v}\right)}^{\mathrm{triv}}(s)\right)=\prod_{v \in V_{\mathrm{SU}}} \zeta_{\mathrm{SU}_{3}\left(\mathbb{F}_{q v}\right)}(s)
$$

is less than or equal to 1.

Proof. Let $q$ be a prime power, not divisible by 3. Explicit formulae for the zeta function of the finite classical group $\mathrm{SU}_{3}\left(\mathbb{F}_{q}\right)$ can be computed, for instance, using Deligne-Lusztig Theory; cf. [40]. One needs to separate the cases $q \equiv_{3} 2$ and $q \equiv_{3} 1$. Indeed, for $q \equiv_{3} 2$ one has

$$
\begin{aligned}
\zeta_{\mathrm{SU}_{3}\left(\mathbb{F}_{q}\right)}(s)=1 & +1 \cdot\left(q^{2}-q\right)^{-s}+q \cdot\left(q^{2}-q+1\right)^{-s} \\
& +6 \cdot\left((q-1)(q+1)^{2} / 3\right)^{-s}+3 \cdot\left(\left(q^{2}-q+1\right)(q-1) / 3\right)^{-s} \\
& +3^{-1}(q+1)(q-2) \cdot\left((q-1)(q+1)^{2}\right)^{-s}+2^{-1}(q+1)(q-2) \cdot\left(q^{3}+1\right)^{-s} \\
& \left.+1 \cdot q^{-3 s}+q \cdot\left(q^{3}-q^{2}+q\right)\right)^{-s} \\
& +6^{-1}(q+1)(q-2) \cdot\left(\left(q^{2}-q+1\right)(q-1)\right)^{-s}
\end{aligned}
$$

and for $q \equiv_{3} 1$ one has

$$
\begin{aligned}
\zeta_{\mathrm{SU}_{3}\left(\mathbb{F}_{q}\right)}(s)=1 & +1 \cdot\left(q^{2}-q\right)^{-s}+q \cdot\left(q^{2}-q+1\right)^{-s} \\
& +3^{-1}\left(q^{2}-q\right) \cdot\left((q-1)(q+1)^{2}\right)^{-s}+2^{-1}(q+1)(q-2) \cdot\left(q^{3}+1\right)^{-s} \\
& +1 \cdot q^{-3 s}+q \cdot\left(q^{3}-q^{2}+q\right)^{-s} \\
& +6^{-1}\left(q^{2}-q\right) \cdot\left(\left(q^{2}-q+1\right)(q-1)\right)^{-s} .
\end{aligned}
$$

Based on these formulae we can prove our assertion. Indeed, $|k: \mathbb{Q}|$ provides a uniform upper bound for the number of valuations $v \notin T$ which prolong any fixed $p$-adic valuation on $\mathbb{Q}$. Moreover, for each $v$ which prolongs the $p$-adic valuation associated to a prime $p$ we have $p \leq q_{v}=\left|\mathcal{O}_{v}: \mathfrak{p}_{v}\right|$. The Chebotarev Density Theorem guarantees that 
TABLE 7.3. Adjoint orbits in $\mathfrak{s u}_{3}\left(\mathbb{F}_{q}\right)$ under the action of $\mathrm{GU}_{3}\left(\mathbb{F}_{q}\right)$

\begin{tabular}{|l|l|l|l|l|}
\hline type & & number of orbits & size of each orbit & total number \\
\hline \hline 0 & & 1 & 1 & 1 \\
1 & reg. & 1 & $\left(q^{3}+1\right)\left(q^{2}-1\right) q$ & $\approx q^{6}$ \\
2 & irreg. & 1 & $\left(q^{3}+1\right)(q-1)$ & $\approx q^{4}$ \\
3 & irreg. & $q-1$ & $\left(q^{2}-q+1\right) q^{2}$ & $\approx q^{5}$ \\
4 a & reg. & $(q-1)(q-2) / 6$ & $\left(q^{2}-q+1\right)(q-1) q^{3}$ & $\approx q^{8}$ \\
$4 \mathrm{~b}$ & reg. & $(q-1) q / 2$ & $\left(q^{3}+1\right) q^{3}$ & $\approx q^{8}$ \\
$4 \mathrm{c}$ & reg. & $\left(q^{2}-1\right) / 3$ & $\left(q^{2}-1\right)(q+1) q^{3}$ & $\approx q^{8}$ \\
5 & reg. & $(q-1)$ & $\left(q^{3}+1\right)(q-1) q^{2}$ & $\approx q^{7}$ \\
\hline
\end{tabular}

TABLE 7.4. Centralisers in $\mathrm{SU}_{3}\left(\mathbb{F}_{q}\right)$ of elements of $\mathfrak{s u}_{3}\left(\mathbb{F}_{q}\right)$ where $q=p^{r}$

\begin{tabular}{|l|l|l|}
\hline type & & centraliser in $\mathrm{SU}_{3}\left(\mathbb{F}_{q}\right)$ \\
\hline \hline 0 & & $\mathrm{SU}_{3}\left(\mathbb{F}_{q}\right)$ \\
1 & reg. & $\left(\mu_{3}\left(\mathbb{F}_{q^{2}}\right) \cap \operatorname{ker}\left(N_{\mathbb{F}_{q^{2}} \mid \mathbb{F}_{q}}\right)\right) \times \mathbb{F}_{q}^{+} \times \mathbb{F}_{q}^{+} \cong C_{\mathrm{gcd}(q+1,3)} \times\left(C_{p}^{r} \times C_{p}^{r}\right)$ \\
2 & irreg. & $\operatorname{ker}\left(N_{\mathbb{F}_{q^{2}} \mid \mathbb{F}_{q}}\right) \ltimes \mathcal{H}\left(\mathbb{F}_{q}\right) \cong C_{q+1} \ltimes\left(C_{p}^{r} \times C_{p}^{r}\right) \ltimes C_{p}^{r}$ \\
3 & irreg. & $\operatorname{GU}_{2}\left(\mathbb{F}_{q}\right)$ \\
$4 \mathrm{a}$ & reg. & $\operatorname{ker}\left(N_{\mathbb{F}_{q^{2}} \mid \mathbb{F}_{q}}\right) \times \operatorname{ker}\left(N_{\mathbb{F}_{q^{2}} \mid \mathbb{F}_{q}}\right) \cong C_{q+1} \times C_{q+1}$ \\
$4 \mathrm{~b}$ & reg. & $\mathbb{F}_{q^{2}}^{*} \cong C_{q^{2}-1}$ \\
$4 \mathrm{c}$ & reg. & $\operatorname{ker}\left(N_{\mathbb{F}_{q^{6}} \mid \mathbb{F}_{q^{3}}}\right) \cap \operatorname{ker}\left(N_{\left.\mathbb{F}_{q^{6}} \mid \mathbb{F}_{q^{2}}\right) \cong C_{q^{2}-q+1}}\right.$ \\
5 & reg. & $\operatorname{ker}\left(N_{\mathbb{F}_{q^{2}} \mid \mathbb{F}_{q}}\right) \ltimes \mathbb{F}_{q}^{+} \cong C_{q+1} \times C_{p}^{r}$ \\
\hline
\end{tabular}

for a positive proportion of primes $p$ there exists a prolongation $v$ such that $p=q_{v}$. In view of the explicit formulae for $\zeta_{\mathrm{SU}_{3}\left(\mathbb{F}_{q}\right)}(s)$ recorded above, this implies that the abscissa of convergence of the product $\prod_{3 \nmid v} \zeta_{\mathrm{SU}_{3}\left(\mathbb{F}_{q_{v}}\right)}(s)$ is equal to the abscissa of convergence of the sum $\sum_{p}\left(p^{1-2 s}+p^{2-3 s}\right)$, which is equal to 1 . This gives an upper bound for the abscissa of convergence of the partial product $\prod_{v \in V_{\mathrm{SU}}} \zeta_{\mathrm{SU}_{3}\left(\mathbb{F}_{q v}\right)}(s)$.

7.4.3. It remains to bound the abscissa of convergence of the factor arising from the last two summands in (7.9).

Proposition 7.12. The abscissa of convergence of the Euler product

$$
\prod_{v \in V_{\mathrm{SU}}}\left(1+\zeta_{\mathrm{SU}_{3}\left(\mathcal{O}_{v}\right)}^{\mathrm{reg}}(s)+\zeta_{\mathrm{SU}_{3}\left(\mathcal{O}_{v}\right)}^{\mathrm{ireg}}(s)\right)
$$

is less than or equal to 1.

The proof of this proposition is based on a similar analysis to the one in Section 7.3.4. By Lemma 7.2 , it suffices to find, for all $v \in V_{\mathrm{SU}}$, approximations $\psi_{v}^{\text {reg }}(s)$ and $\psi_{v}^{\text {irreg }}(s)$ so that, locally,

$$
\zeta_{\mathrm{SU}_{3}\left(\mathcal{O}_{v}\right)}^{\mathrm{reg}}(s) \ll \psi_{v}^{\mathrm{reg}}(s) \quad \text { and } \quad \zeta_{\mathrm{SU}_{3}\left(\mathcal{O}_{v}\right)}^{\mathrm{irreg}}(s) \ll \psi_{v}^{\text {irreg }}(s)
$$

and, globally, the abscissa of convergence of the $\operatorname{sum} \sum_{v \in V_{\mathrm{SU}}}\left(\psi_{v}^{\mathrm{reg}}(s)+\psi_{v}^{\text {irreg }}(s)\right)$ is at most 1 .

In order to apply approximative Clifford theory, we require an overview of the elements in $\mathfrak{s u}_{3}\left(\mathbb{F}_{q}\right)$ up to the adjoint action of the group $\mathrm{GU}_{3}\left(\mathbb{F}_{q}\right)$. We distinguish eight different types, labelled $0,1,2,3,4 \mathrm{a}, 4 \mathrm{~b}, 4 \mathrm{c}, 5$. The total number of elements of each type and 
the isomorphism types of their centralisers in $\mathrm{SU}_{3}\left(\mathbb{F}_{q}\right)$ are summarised in Tables 7.3 and 7.4: see Appendix $\mathrm{C}$ for a short discussion. The notation used in Table 7.4 is similar to the one in Table 7.2 , $\mu_{3}\left(\mathbb{F}_{q^{2}}\right)$ is the group of third roots of unity in $\mathbb{F}_{q^{2}}^{*}$, we denote by $\mathcal{H}\left(\mathbb{F}_{q}\right)$ the Heisenberg group over $\mathbb{F}_{q}$, etc.

Let $v$ be a place of $k$ with $v \in V_{\mathrm{SU}}$. The series $\psi_{v}^{\text {reg }}(s)$ is to approximate the second summand in (7.9). It splits into smaller parts

$$
\begin{aligned}
\psi_{v}^{\mathrm{reg}}(s) & =\psi_{v}^{1}(s)+\psi_{v}^{4 \mathrm{a}}(s)+\psi_{v}^{4 \mathrm{~b}}(s)+\psi_{v}^{4 \mathrm{c}}(s)+\psi_{v}^{5}(s) \\
& =\psi_{v}^{1(\mathrm{a})}(s)+\psi_{v}^{1(\mathrm{~b})}(s)+\psi_{v}^{4 \mathrm{a}}(s)+\psi_{v}^{4 \mathrm{~b}}(s)+\psi_{v}^{4 \mathrm{c}}(s)+\psi_{v}^{5}(s),
\end{aligned}
$$

which correspond to elements of types $1,4 \mathrm{a}, 4 \mathrm{~b}, 4 \mathrm{c}$ and 5 in the finite Lie algebra $\mathfrak{s u}_{3}\left(\mathbb{F}_{q}\right)$ and where $\psi_{v}^{1}(s)$ consists of two summands, following a case distinction similar to the analogous one in Section 7.3.4 Each of the summands involved will be a rational function in $q_{v}, q_{v}^{-s}$ and $2^{-s}$, which we derive using Clifford theory. The series $\psi_{v}^{\text {irreg }}(s)$ is to approximate the third summand in (7.9). It splits into smaller parts

$$
\begin{aligned}
\psi_{v}^{\text {irreg }}(s) & =\psi_{v}^{2}(s)+\psi_{v}^{3}(s) \\
& =\psi_{v}^{2(\mathrm{a})}(s)+\psi_{v}^{2(\mathrm{~b})}(s)+\psi_{v}^{2(\mathrm{c})}(s)+\psi_{v}^{3(\mathrm{a})}(s)+\psi_{v}^{3(\mathrm{~b})}(s),
\end{aligned}
$$

which correspond to elements of types 2 and 3 in $\mathfrak{s u}_{3}\left(\mathbb{F}_{q_{v}}\right)$ and where $\psi_{v}^{2}(s)$ and $\psi_{v}^{3}(s)$ divide into smaller summands, following a case distinction similar to the analogous one in Section 7.3.4. Again, each of these summands will be a rational function in $q_{v}, q_{v}^{-s}$ and $2^{-s}$, which can be obtained by means of Clifford theory.

7.4.4. We now work out the details case by case. Let $v$ be a place of $k$ with $v \in V_{\mathrm{SU}}$. In describing the local situation at $v$, it is convenient to adopt, as in Section 7.3.4, a simplified notation which suppresses explicit references to $v$. We write $\mathfrak{o}:=\mathcal{O}_{v}, \mathfrak{p}:=\mathfrak{p}_{v}$ for the maximal ideal and $\mathbb{F}_{q}:=\mathfrak{o} / \mathfrak{p}$ for the residue field, where $q:=q_{v}$. We denote by $\mathfrak{O}$ an unramified quadratic extension of $\mathfrak{o}$, with non-trivial automorphism $\sigma$. Furthermore, we write $\psi_{\mathfrak{o}}^{1}(s):=\psi_{v}^{1}(s)$, etc. We also continue to use the simplified notation $\mathrm{GU}_{3}(\mathfrak{o}):=$ $\mathrm{GU}_{3}(\mathfrak{O}, \mathfrak{o})$, etc.

One sets $G:=\mathrm{SU}_{3}(\mathfrak{o}), N:=\mathrm{SU}_{3}^{1}(\mathfrak{o})$ and $\gamma(q):=q^{-8}|G: N|=\left(1+q^{-3}\right)\left(1-q^{-2}\right)$. We use the generous bound $\gamma(q)>2^{-1}$. In each case we need to multiply the approximate contribution number from the last column of Table 7.3, an approximation of the translation factor $\left|G: I_{G}(\vartheta)\right|^{-1-s} \zeta_{G, \vartheta}(s)$, in accordance with (7.2), and (an approximation of) the appropriate series factor, appearing on one of the right hand sides of (6.6). As in Section 7.3.4, in the case of regular representations we use the precise translation factor $\left(1-q^{2-3 s}\right)^{-1}$; for irregular representations we use the generous approximative factor $\left(1+q^{4-2 s}\right)\left(1-q^{1-2 s}\right)^{-1}\left(1-q^{2-3 s}\right)^{-1}$. As the detailed analysis is very similar to the one carried out for inner forms, we only give a short indication of the necessary modifications and we list the resulting approximative Dirichlet generating functions. The most involved case is once more the one of type 3 ; here our adaptation makes use of the Cayley map. We deal with the different cases arising from Table 7.3 in the following order: 4a, 4b, 4c, 5, 2, 1, 3 .

Types 4a, 4b, 4c. The changes for type 4 are easy to make. Using Corollary 7.7, instead of Corollary 7.6, we compute

$$
\psi_{\mathfrak{o}}^{4 \mathrm{a}}(s)=q^{8} \cdot 2^{3+2 s} q^{-4-6 s} \cdot\left(1-q^{2-3 s}\right)^{-1}=2^{3+2 s} q^{4-6 s}\left(1-q^{2-3 s}\right)^{-1},
$$

to control the contribution from elements of type $4 \mathrm{a}$, and one uses similar estimates for types $4 \mathrm{~b}$ and $4 \mathrm{c}$. 
Type 5. Also the changes for type 5 are straightforward. Using Corollary 7.7, instead of Corollary 7.6, we compute

$$
\psi_{\mathfrak{o}}^{5}(s)=q^{7} \cdot 2^{3+2 s} q^{-4-6 s} \cdot\left(1-q^{2-3 s}\right)^{-1}=2^{3+2 s} q^{3-6 s}\left(1-q^{2-3 s}\right)^{-1}
$$

to control the contribution from elements of type 5 .

Type 2. We explain briefly how to adopt the argument for elements of type 2. According to Table 7.4, the inertia quotient $T_{G}(\vartheta) / N$ embeds into the semidirect product $\operatorname{ker}\left(N_{\mathbb{F}_{q^{2}} \mid \mathbb{F}_{q}}\right) \ltimes \mathcal{H}\left(\mathbb{F}_{q}\right)$, and the analysis beginning at (C.2) shows that the top group $\operatorname{ker}\left(N_{\mathbb{F}_{q^{2}} \mid \mathbb{F}_{q}}\right) \cong C_{q+1}$ acts with kernel of size $\operatorname{gcd}(q+1,3)$ and with orbits of equal length $(q+1) / \operatorname{gcd}(q+1,3)$ on the non-trivial elements of $\mathcal{H}\left(\mathbb{F}_{q}\right) /\left[\mathcal{H}\left(\mathbb{F}_{q}\right), \mathcal{H}\left(\mathbb{F}_{q}\right)\right]$. Arguing as before, we compute

$$
\begin{aligned}
\psi_{\mathfrak{o}}^{2(\mathrm{a})}(s) & =q^{4} \cdot 2^{3+2 s}\left(10 q^{-3-4 s}+q^{-2-5 s}\right) \cdot\left(1+q^{4-2 s}\right)\left(1-q^{1-2 s}\right)^{-1}\left(1-q^{2-3 s}\right)^{-1} \\
& =2^{3+2 s}\left(10 q^{1-4 s}+10 q^{5-6 s}+q^{2-5 s}+q^{6-7 s}\right)\left(1-q^{1-2 s}\right)^{-1}\left(1-q^{2-3 s}\right)^{-1}, \\
\psi_{\mathfrak{o}}^{2(\mathrm{~b})}(s) & =q^{4} \cdot 2 q^{3} \cdot 2^{2+2 s} q^{-5-5 s} \cdot\left(1+q^{4-2 s}\right)\left(1-q^{1-2 s}\right)^{-1}\left(1-q^{2-3 s}\right)^{-1} \\
& =2^{3+2 s}\left(q^{2-5 s}+q^{6-7 s}\right)\left(1-q^{1-2 s}\right)^{-1}\left(1-q^{2-3 s}\right)^{-1}, \\
\psi_{\mathfrak{o}}^{2(\mathrm{c})}(s) & =q^{4} \cdot 2 q^{4} \cdot q^{-2-s} \cdot 2^{2+2 s} q^{-4-4 s} \cdot\left(1+q^{4-2 s}\right)\left(1-q^{1-2 s}\right)^{-1}\left(1-q^{2-3 s}\right)^{-1} \\
& =2^{3+2 s}\left(q^{2-5 s}+q^{6-7 s}\right)\left(1-q^{1-2 s}\right)^{-1}\left(1-q^{2-3 s}\right)^{-1}
\end{aligned}
$$

to control the contribution from elements of type 2 .

Type 1. The changes for type 1 are easy to make. Using Corollary 7.7, instead of Corollary 7.6, we compute the same approximative Dirichlet generating functions

$$
\begin{aligned}
& \psi_{\mathfrak{o}}^{1(\mathrm{a})}(s)=q^{6} \cdot 2^{3+s} q^{-4-6 s} \cdot\left(1-q^{2-3 s}\right)^{-1}=2^{3+s} q^{2-6 s}\left(1-q^{2-3 s}\right)^{-1}, \\
& \psi_{\mathfrak{o}}^{1(\mathrm{~b})}(s)=q^{6} \cdot 4 q^{2} \cdot q^{-2-s} \cdot 2^{1+s} q^{-6-6 s} \cdot\left(1-q^{2-3 s}\right)^{-1}=2^{3+s} q^{-7 s}\left(1-q^{2-3 s}\right)^{-1} .
\end{aligned}
$$

to control the contribution from elements of type 1 .

Type 3 . The argument for type 3 requires more substantial modifications. The number of elements is bounded by $q^{5}$, and the difficulty is that the precise inertia group quotient is typically significantly smaller than $\mathrm{GU}_{2}\left(\mathbb{F}_{q}\right)$, the supergroup supplied by Table 7.4 . In Section 7.3 .4 where we dealt with inner forms, the associative algebra $\operatorname{Mat}_{3}(\mathfrak{o})$ was used to translate between the compact analytic group $\mathrm{SL}_{3}(\mathfrak{o})$ and the $\mathfrak{o}$-Lie lattice $\mathfrak{s l}_{3}(\mathfrak{o})$. In the situation at hand, there is no associative algebra which connects $G=\operatorname{SU}_{3}(\mathfrak{o})$ and $\mathfrak{g}=\mathfrak{s u}_{3}(\mathfrak{o})$ at the level of $\mathfrak{o}$, but one can argue via the Cayley maps; see [52, II.10 and VI.2]. For any subset $Y \subseteq \operatorname{Mat}_{3}(\mathfrak{O})$, we denote by $Y_{\text {gen }}$ the set of elements of $Y$ which do not have an eigenvalue congruent to -1 modulo $\mathfrak{p}$. The Cayley maps Cay: $\mathrm{GU}_{3}(\mathfrak{o})_{\text {gen }} \rightarrow \mathfrak{g u}_{3}(\mathfrak{o})_{\text {gen }}$ and cay $: \mathfrak{g u}_{3}(\mathfrak{o})_{\text {gen }} \rightarrow \mathrm{GU}_{3}(\mathfrak{o})_{\text {gen }}$ which are each defined by the mapping rule

$$
y \mapsto(1-y)(1+y)^{-1}=(1+y)^{-1}(1-y)
$$

are easily seen to be mutual inverses of each other; cf. Appendix C.1.

As in Section 7.3.4 fix $x \in \mathfrak{n}^{*}$ and a level $n \in \mathbb{N}$ such that $I_{G}(\vartheta)=\mathrm{C}_{G}\left(x+\mathfrak{p}^{n} \mathfrak{n}\right) N$. From the definition of the Cayley maps it is clear that we have inclusions

$$
\mathrm{C}_{\mathrm{GU}_{3}(\mathfrak{o})}\left(x+\mathfrak{p}^{n} \mathfrak{n}\right)_{\text {gen }} \stackrel{\text { Cay }}{\longrightarrow} \mathrm{C}_{\mathfrak{g u}_{3}(\mathfrak{o})}\left(x+\mathfrak{p}^{n} \mathfrak{n}\right)_{\text {gen }} \stackrel{\text { cay }}{\longrightarrow} \mathrm{C}_{\mathrm{GU}_{3}(\mathfrak{o})}\left(x+\mathfrak{p}^{n} \mathfrak{n}\right)_{\text {gen }} .
$$

We need to translate these inclusions into a quantitative assertion about the cardinalities of the inertia group quotient $I_{G}(\vartheta) / N=\mathrm{C}_{\mathrm{SU}_{3}(\mathfrak{o})}\left(x+\mathfrak{p}^{n} \mathfrak{n}\right) N / N$ and the Lie lattice quotient $\left(\mathrm{C}_{\mathfrak{s u}_{3}(\mathfrak{o})}\left(x+\mathfrak{p}^{n} \mathfrak{n}\right)+\mathfrak{n}\right) / \mathfrak{n}$. 
Elementary considerations, using $q \geq 5$, show that there exists $\lambda \in \mathfrak{O}^{*}$ with $\lambda+\lambda^{\sigma}=0$ such that $\mathfrak{g u}_{3}(\mathfrak{o}) \subseteq \mathfrak{g u}_{3}(\mathfrak{o})_{\text {gen }} \cup\left(\mathfrak{g u}_{3}(\mathfrak{o})+\lambda \text { Id }\right)_{\text {gen }}$. This implies that

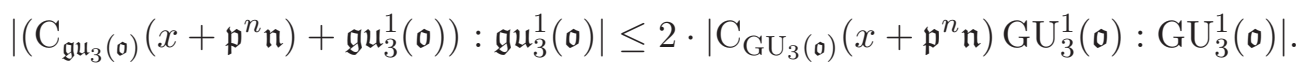

Similarly, there exist $\lambda_{1}, \ldots, \lambda_{4} \in \mathfrak{O}^{*}$ with $\lambda_{i} \lambda_{i}^{\sigma}=1$ such that

$$
\mathrm{GU}_{3}(\mathfrak{o}) \subseteq \bigcup_{i=1}^{4}\left(\lambda_{i} \mathrm{GU}_{3}(\mathfrak{o})\right)_{\text {gen }}
$$

This implies that

$$
\left.4 \cdot \mid \mathrm{C}_{\mathfrak{g u}_{3}(\mathfrak{o})}\left(x+\mathfrak{p}^{n} \mathfrak{n}\right)+\mathfrak{g u} \mathfrak{u}_{3}^{1}(\mathfrak{o})\right): \mathfrak{g} \mathfrak{u}_{3}^{1}(\mathfrak{o})|\geq| \mathrm{C}_{\mathrm{GU}_{3}(\mathfrak{o})}\left(x+\mathfrak{p}^{n} \mathfrak{n}\right) \mathrm{GU}_{3}^{1}(\mathfrak{o}): \operatorname{GU}_{3}^{1}(\mathfrak{o}) \mid .
$$

At the same time we have

$$
\left|\mathrm{C}_{\mathfrak{g u}}(\mathfrak{o})\left(x+\mathfrak{p}^{n} \mathfrak{n}\right)+\mathfrak{g} \mathfrak{u}_{3}^{1}(\mathfrak{o}): \mathrm{C}_{\mathfrak{s u}(\mathfrak{o})}\left(x+\mathfrak{p}^{n} \mathfrak{n}\right)+\mathfrak{g} \mathfrak{u}_{3}^{1}(\mathfrak{o})\right|=q
$$

and

$$
q+1 \geq\left|\mathrm{C}_{\mathrm{GU}_{3}(\mathfrak{o})}\left(x+\mathfrak{p}^{n} \mathfrak{n}\right) \mathrm{GU}_{3}^{1}(\mathfrak{o}): \mathrm{C}_{\mathrm{SU}_{3}(\mathfrak{o})}\left(x+\mathfrak{p}^{n} \mathfrak{n}\right) \mathrm{GU}_{3}^{1}(\mathfrak{o})\right| \geq \frac{q+1}{\operatorname{gcd}(q+1,3)} .
$$

Altogether we thus obtain the generous estimates

$$
4^{-1} \leq \frac{\left|\mathrm{C}_{\mathrm{SU}_{3}(\mathfrak{o})}\left(x+\mathfrak{p}^{n} \mathfrak{n}\right) N / N\right|}{\left|\left(\mathrm{C}_{\mathfrak{s u}_{3}(\mathfrak{o})}\left(x+\mathfrak{p}^{n} \mathfrak{n}\right)+\mathfrak{n}\right) / \mathfrak{n}\right|} \leq 12 .
$$

With this preparation, we now distinguish two cases, as for elements of type 3 in the setting of inner forms. In the first case, $x+\mathfrak{p}^{n} \mathfrak{n}$ corresponds to a point modulo $\mathfrak{p}^{n}$ on the variety $\mathcal{W}_{1}$ defined by the set $F_{3}(\mathbf{Y})$ of polynomials which controls the integrand. These are rare and lead to the effective 'series factor' $1 /\left(1-q^{1-2 s}\right)$. The inertia group quotient is isomorphic to $\mathrm{GU}_{2}\left(\mathbb{F}_{q}\right)$ so that $\left|G: I_{G}(\vartheta)\right|=q^{2}\left(q^{2}-q+1\right) \geq 2^{-1} q^{4}$. A similar argument as before shows that $\vartheta$ extends to $I_{G}(\vartheta)$ so that

$$
\begin{aligned}
\zeta_{G, \vartheta}(s) & =\zeta_{\mathrm{GU}_{2}\left(\mathbb{F}_{q}\right)}(s) \\
& =(q+1)\left(1+q^{-s}+(q-2) / 2 \cdot(q+1)^{-s}+q / 2 \cdot(q-1)^{-s}\right) \\
& \ll 2 q+2^{1+s} q^{2-s} .
\end{aligned}
$$

Thus the contributing summand is

$$
\begin{aligned}
\psi_{\mathfrak{o}}^{3(\mathrm{a})}(s) & =q^{5} \cdot\left(2 q+2^{1+s} q^{2-s}\right) \cdot 2^{1+s} q^{-4-4 s} \cdot\left(1-q^{1-2 s}\right)^{-1} \\
& =\left(q^{2-4 s}+2^{1+s} q^{3-5 s}\right)\left(1-q^{1-2 s}\right)^{-1} .
\end{aligned}
$$

In the second case,

$$
\left|I_{G}(\vartheta): N\right| \leq 12 \cdot\left|\left(\mathrm{C}_{\mathfrak{s u}(\mathfrak{o})}\left(x+\mathfrak{p}^{n} \mathfrak{n}\right)+\mathfrak{n}\right): \mathfrak{n}\right| \leq 12 q^{2},
$$

and we approximate $\zeta_{I_{G}(\vartheta) / N}(s)$ generously by $2^{4} q^{2}$. Bounding $\left|G: I_{G}(\vartheta)\right|$ from below by $\gamma(q) 2^{-4} q^{6}>2^{-5} q^{6}$ and approximating the required series factor by $\left(1+q^{4-2 s}\right)(1-$ $\left.q^{1-2 s}\right)^{-1}\left(1-q^{2-3 s}\right)^{-1}$, we obtain the contribution

$$
\begin{aligned}
\psi_{\mathfrak{o}}^{3(\mathrm{~b})}(s) & =q^{5} \cdot 2^{4} q^{2} \cdot 2^{5+5 s} q^{-6-6 s} \cdot\left(1+q^{4-2 s}\right)\left(1-q^{1-2 s}\right)^{-1}\left(1-q^{2-3 s}\right)^{-1} \\
& =2^{9+5 s}\left(q^{1-6 s}+q^{5-8 s}\right)\left(1-q^{1-2 s}\right)^{-1}\left(1-q^{2-3 s}\right)^{-1} .
\end{aligned}
$$

As in in Section 7.3.4, direct inspection shows that the estimates provided for the contributions of the various types justify Proposition 7.12. Together with Proposition 7.11 and the reductions leading up to these two propositions, this completes the proof of Theorem $\mathrm{C}$ in the case of outer forms. 


\section{Appendices}

\section{Appendix A. Algebraic groups of type $A_{2}$}

Let $n \in \mathbb{N}$. We are interested in arithmetic subgroups $\Gamma$ of semisimple algebraic groups, in particular simple algebraic groups of type $A_{2}$. These groups arise in the following way. Let $\mathbf{G}$ be a connected, simply-connected semisimple algebraic group defined over a number field $k$, together with a fixed $k$-embedding into $\mathrm{GL}_{N}$ for some $N \in \mathbb{N}$. Let $\mathcal{O}_{S}$ denote the ring of $S$-integers in $k$, for a finite set $S$ of places of $k$ including all the archimedean ones. We consider groups $\Gamma$ which are commensurable to $\mathbf{G}\left(o_{S}\right)=\mathbf{G}(k) \cap \mathrm{GL}_{n}\left(o_{S}\right)$.

In this paper we are concerned with groups $\mathbf{G}$ which are simple of type $A_{n}$, for the special case $n=2$. These groups are subdivided into groups of types ${ }^{1} A_{n}$ (pertaining to inner forms) and ${ }^{2} A_{n}$ (pertaining to outer forms) over $k$. Based on [43, Propositions 2.17 and 2.18], we extract from the general classification of simple algebraic groups of type $A_{n}$ the following information relevant to the special case of interest to us.

The classification allows for the following groups of type ${ }^{1} A_{2}$ over the number field $k$ :

(1) $\mathbf{G}(k)=\mathrm{SL}_{3}(k)$,

(2) $\mathbf{G}(k)=\mathrm{SL}_{1}(D)$ where $D$ is a central division algebra of index 3 over $k$.

If $\Gamma=\mathbf{G}\left(\mathcal{O}_{S}\right)$ for either one of these $k$-defined algebraic groups, then for almost all places $v$, with $v \notin S$, the local group $\Gamma_{v}=\mathbf{G}\left(\mathcal{O}_{v}\right)$ is isomorphic to $\mathrm{SL}_{3}\left(\mathcal{O}_{v}\right)$. For the finitely many exceptional places $v$ the local group $\Gamma_{v}$ is commensurable to either $\mathrm{SL}_{3}\left(\mathcal{O}_{v}\right)$ or $\mathrm{SL}_{1}(\mathfrak{D})$ where $\mathfrak{D}$ is the (unique) division algebra of index 2 over $k_{v}$.

Similarly, the classification allows for the following groups of type ${ }^{2} A_{2}$ over the number field $k$ :

(1) $\mathrm{SU}_{3}(K, f)=\left\{g \in \mathrm{GL}_{3}(K) \mid \operatorname{det}(g)=1\right.$ and $\left.g^{\circ} H g=H\right\}$ where $K=k(\sqrt{\delta})$, $\delta \in \mathcal{O}$ not a square, is a quadratic field extension with non-trivial Galois automorphism $\sigma$, the operation $\circ$ is the $K \mid k$-involution 'conjugate transpose' of the central algebra $A=\operatorname{Mat}_{3}(K)$ over $K$, given by $\mathbf{a}^{\circ}=\left(\mathbf{a}^{\sigma}\right)^{\mathrm{t}}$, and $H \in \operatorname{Mat}_{3}(K)$ is the structure matrix of a non-degenerate Hermitian sesquilinear form $f$ on $V=K^{3}$

(2) $\mathrm{SU}_{1}(D, f)=\left\{g \in \mathrm{GL}_{1}(D) \mid \operatorname{Nrd}_{D \mid K}(g)=1\right.$ and $\left.g^{\tau} \eta g=\eta\right\}$ where $K=k(\sqrt{\delta})$, $\delta \in \mathcal{O}$ not a square, is a quadratic field extension, $A=D$ is a central division algebra of index 3 over $K$ with a $K \mid k$-involution $\tau$, and $\eta \in D$ with $\eta^{\tau}=\eta$.

Suppose that $\Gamma=\mathbf{G}\left(\mathcal{O}_{S}\right)$ for either one of these $k$-defined algebraic groups, defined in terms of a central simple algebra $A$ with $K \mid k$-involution. By [45, Remark 6.6.3 and Theorem 10.2.5] there are essentially two types of completions $A_{v}$ of $A$, as $v$ runs over places of $k$ not in $S$. For places $v \notin S$ of $k$ which are non-decomposed in $K \mid k$, the completion of the $k$-algebra $K$ at $v$ yields a quadratic field extension $K_{v} \mid k_{v}$ and, apart from finitely many exceptional places, $A_{v}$ is isomorphic to $\operatorname{Mat}_{3}\left(K_{v}\right)$ equipped with the standard $K_{v} \mid k_{v}$-involution. In this situation, excluding again finitely many places, we have $\Gamma_{v} \cong \mathrm{SU}_{3}\left(\mathcal{O}_{v}\right)$, where $\mathrm{SU}_{3}\left(\mathcal{O}_{v}\right):=\mathrm{SU}_{3}\left(\mathfrak{O}, \mathcal{O}_{v}\right)$ with $\mathfrak{O}$ denoting an unramified quadratic extension of $\mathcal{O}_{v}$. For places $v \notin S$ of $k$ which are decomposed in $K \mid k$ the completion $K_{v}$ is isomorphic to $k_{v} \times k_{v}$ and, apart from finitely many exceptions, $A$ is isomorphic to $\operatorname{Mat}_{3}\left(k_{v}\right) \times \operatorname{Mat}_{3}\left(k_{v}\right)$ with the involution $(X, Y) \rightarrow\left(Y^{\mathrm{t}}, X^{\mathrm{t}}\right)$. In this situation, excluding again finitely many places, we have $\Gamma_{v} \cong \mathrm{SL}_{3}\left(\mathcal{O}_{v}\right)$.

For $v \notin S$, let $q_{v}$ denote the residue field cardinality of $\mathcal{O}_{v}$, and define the sets of rational primes $P_{\mathrm{SL}}:=\left\{p \mid \exists v \notin S: \Gamma_{v} \cong \mathrm{SL}_{3}\left(\mathcal{O}_{v}\right)\right.$ and $p=q_{v}$ is prime $\}$ and $P_{\mathrm{SU}}:=\{p \mid$ 
$\exists v \notin S: \Gamma_{v} \cong \mathrm{SU}_{3}\left(\mathcal{O}_{v}\right)$ and $p=q_{v}$ is prime\}. In connection with our approach toward outer forms in Section [7.4, e.g. in the proof of Proposition 7.11, the following lemma is noteworthy.

Lemma A.1. Each of the sets $P_{\mathrm{SL}}$ and $P_{\mathrm{SU}}$ has positive Dirichlet density within the set of all primes.

Proof. In both cases the assertion is a consequence of the Chebotarev Density Theorem. In the case of $P_{\mathrm{SL}}$ the argument is straightforward; the set of primes $p$ which are totally split in $K$ has positive density.

In order to show that $P_{\mathrm{SU}}$ has positive density, it suffices to supply a positive proportion of primes $p$ which admit a prolongation $v$ to $k$ such that $k_{v}$ has residue field of size $q_{v}=p$ and such that $v$ is inert in the quadratic extension $K \mid k$. For simplicity, we consider all number fields to be subfields of a fixed algebraic closure of $\mathbb{Q}$, e.g. within $\mathbb{C}$.

We distinguish two cases. First suppose that $K$ is not contained in the normal closure $N$ of $k$. Then the normal closure of $K$ has degree 2 over $N$, and the Chebotarev Density Theorem shows that the proportion of primes $p$ which are totally split in $k$, viz. $|N: \mathbb{Q}|^{-1}$, is positive and twice as much as the proportion of primes $p$ which are totally split in $K$. Apart from finitely many exceptions, these primes are of the desired kind.

We now turn to the more interesting case that $K$ is contained in the normal closure $N$ of $k$. In the Galois group $G=\operatorname{Gal}(N \mid \mathbb{Q})$, let $H_{k}$ denote the subgroup consisting of automorphisms which fix element-wise $k$, and let $H_{K}$ be the subgroup consisting of automorphisms which fix element-wise $K$. Then $H_{K} \unlhd H_{k}$ with $\left|H_{k}: H_{K}\right|=2$. Choose $h \in H_{k}$ such that $H_{k}=\langle h\rangle H_{K}$, and let $C$ denote the conjugacy class of $h$ in $G$. The Chebotarev Density Theorem supplies a positive proportion of primes $p$, unramified in $N \mid \mathbb{Q}$, such that the Frobenius conjugacy class of $p$ in $G$ is equal to $C$. Fix one of these primes $p$, let $w=w_{N}$ be a prolongation of $p$ to $N$, and write $w_{K}:=\left.w\right|_{K}$ and $v:=\left.w\right|_{k}$ for the restrictions of $w$ to $K$ and $k$. Then the decomposition group $G_{w}$ of $w$ maps isomorphically onto the cyclic Galois group of the unramified extension $N_{w} \mid \mathbb{Q}_{p}$. Without loss of generality let us assume that $G_{w}=\langle h\rangle$. As $h$ acts as the identity on $k$, we have $\left|k_{v}: \mathbb{Q}_{p}\right|=1$, i.e. $q_{v}=p$, and as $h$ does not act as the identity on $K$, we must have $\left|K_{w_{K}}: k_{v}\right|=2$. Thus $p$ is of the desired kind.

\section{Appendix B. Adjoint ACtion OF $\mathrm{GL}_{3}\left(\mathbb{F}_{q}\right)$ ON $\mathfrak{s l}_{3}\left(\mathbb{F}_{q}\right)$}

Let $\mathbb{F}_{q}$ be a finite field of characteristic not equal to 3. In Section 7.3 , we require an overview of the elements in $\mathfrak{s l}_{3}\left(\mathbb{F}_{q}\right)$ up to conjugacy under the group $\mathrm{GL}_{3}\left(\mathbb{F}_{q}\right)$. We distinguish eight different types, labelled 0, 1, 2, 3, 4a, 4b, 4c, 5. The total number of elements of each type and the isomorphism types of their centralisers in $\mathrm{SL}_{3}\left(\mathbb{F}_{q}\right)$ are summarised in Tables 7.1 and 7.2. We briefly discuss the eight different types.

Type 0 consists of the zero matrix, which does not feature in our calculation but is shown for completeness. Its centraliser is the entire group $\mathrm{SL}_{3}\left(\mathbb{F}_{q}\right)$.

Type 1 consists of nilpotent matrices with minimal polynomial equal to $X^{3}$ over $\mathbb{F}_{q}$. The centraliser of a typical element is

$$
\mathrm{C}_{\mathrm{SL}_{3}\left(\mathbb{F}_{q}\right)}\left(\left(\begin{array}{lll}
0 & 1 & 0 \\
0 & 0 & 1 \\
0 & 0 & 0
\end{array}\right)\right)=\left\{\left(\begin{array}{lll}
a & b & c \\
0 & a & b \\
0 & 0 & a
\end{array}\right) \in \mathrm{GL}_{3}\left(\mathbb{F}_{q}\right) \mid a^{3}=1\right\}
$$

and matrices of type 1 are regular.

Type 2 consists of nilpotent matrices with minimal polynomial equal to $X^{2}$ over $\mathbb{F}_{q}$. The centraliser of a typical element is

$$
\mathrm{C}_{\mathrm{SL}_{3}\left(\mathbb{F}_{q}\right)}\left(\left(\begin{array}{lll}
0 & 1 & 0 \\
0 & 0 & 0 \\
0 & 0 & 0
\end{array}\right)\right)=\left\{\left(\begin{array}{lll}
a & b & c \\
0 & a & 0 \\
0 & d & e
\end{array}\right) \in \mathrm{GL}_{3}\left(\mathbb{F}_{q}\right) \mid a^{2} e=1\right\}
$$


and matrices of type 2 are irregular.

Type 3 consists of semisimple matrices with eigenvalues $\lambda \in \mathbb{F}_{q} \backslash\{0\}$ of multiplicity 2 and $\mu:=-2 \lambda$. The minimal polynomial of such elements over $\mathbb{F}_{q}$ is equal to $(X-$ $\lambda)(X-\mu)$. The centraliser of a typical element is

$$
\mathrm{C}_{\mathrm{SL}_{3}\left(\mathbb{F}_{q}\right)}\left(\left(\begin{array}{ccc}
\lambda & 0 & 0 \\
0 & \lambda & 0 \\
0 & 0 & \mu
\end{array}\right)\right)=\left\{\left(\begin{array}{ccc}
a & b & 0 \\
c & d & 0 \\
0 & 0 & e
\end{array}\right) \in \mathrm{GL}_{3}\left(\mathbb{F}_{q}\right) \mid(a d-b c) e=1\right\}
$$

and matrices of type 3 are irregular.

Type $4 \mathrm{a}$ consists of semisimple matrices with distinct eigenvalues $\lambda, \mu, \nu:=-\lambda-\mu \in$ $\mathbb{F}_{q} \backslash\{0\}$. The minimal polynomial of such elements over $\mathbb{F}_{q}$ is equal to $(X-\lambda)(X-$ $\mu)(X-\nu)$. The centraliser of a typical element is

$$
\mathrm{C}_{\mathrm{SL}_{3}\left(\mathbb{F}_{q}\right)}\left(\left(\begin{array}{lll}
\lambda & 0 & 0 \\
0 & \mu & 0 \\
0 & 0 & \nu
\end{array}\right)\right)=\left\{\left(\begin{array}{lll}
a & 0 & 0 \\
0 & b & 0 \\
0 & 0 & c
\end{array}\right) \in \mathrm{GL}_{3}\left(\mathbb{F}_{q}\right) \mid a b c=1\right\}
$$

and matrices of type $4 \mathrm{a}$ are regular.

Type $4 \mathrm{~b}$ consists of semisimple matrices with eigenvalues $\lambda, \mu:=\lambda^{q} \in \mathbb{F}_{q^{2}} \backslash \mathbb{F}_{q}$ and $\nu:=-\lambda-\mu \in \mathbb{F}_{q}$. The minimal polynomial of such elements over $\mathbb{F}_{q}$ is equal to $(X-\lambda)(X-\mu)(X-\nu)$. The centraliser of a typical element is isomorphic to the multiplicative group of the field $\mathbb{F}_{q^{2}}$ and matrices of type $4 \mathrm{~b}$ are regular.

Type $4 \mathrm{c}$ consists of semisimple matrices with eigenvalues $\lambda, \mu:=\lambda^{q}, \nu:=\lambda^{q^{2}} \in \mathbb{F}_{q^{3}} \backslash \mathbb{F}_{q}$ with $\lambda+\mu+\nu=0$. The minimal polynomial of such elements over $\mathbb{F}_{q}$ is equal to $(X-\lambda)(X-\mu)(X-\nu)$. The centraliser of a typical element is isomorphic to the group of elements of norm 1 in the field $\mathbb{F}_{q^{3}}$ and matrices of type $4 \mathrm{c}$ are regular.

Type 5 consists of matrices with eigenvalues $\lambda \in \mathbb{F}_{q} \backslash\{0\}$ of multiplicity 2 and $\mu:=$ $-2 \lambda$. The minimal polynomial of such elements over $\mathbb{F}_{q}$ is equal to $(X-\lambda)^{2}(X-\mu)$. The centraliser of a typical element is

$$
\mathrm{C}_{\mathrm{SL}_{3}\left(\mathbb{F}_{q}\right)}\left(\left(\begin{array}{ccc}
\lambda & 1 & 0 \\
0 & \lambda & 0 \\
0 & 0 & \mu
\end{array}\right)\right)=\left\{\left(\begin{array}{ccc}
a & b & 0 \\
0 & a & 0 \\
0 & 0 & c
\end{array}\right) \in \mathrm{GL}_{3}\left(\mathbb{F}_{q}\right) \mid a^{2} c=1\right\}
$$

and matrices of type 5 are regular.

\section{Appendix C. Adjoint action of $\mathrm{GU}_{3}\left(\mathbb{F}_{q^{2}}, \mathbb{F}_{q}\right)$ On $\mathfrak{s u}_{3}\left(\mathbb{F}_{q^{2}}, \mathbb{F}_{q}\right)$}

In Section 7.3, we require an overview of the elements in $\mathfrak{s u}_{3}\left(\mathbb{F}_{q^{2}}, \mathbb{F}_{q}\right)$ up to conjugacy under the group $\mathrm{GU}_{3}\left(\mathbb{F}_{q^{2}}, \mathbb{F}_{q}\right)$. We assume that the characteristic of $\mathbb{F}_{q}$ is at least 5 , and for the purpose of explicit computations we write $\mathbb{F}_{q^{2}}=\mathbb{F}_{q}(\sqrt{\rho})$, where $\rho \in \mathbb{F}_{q}$ is not a square. We distinguish eight different types, labelled $0,1,2,3,4 \mathrm{a}, 4 \mathrm{~b}, 4 \mathrm{c}, 5$. The total number of elements of each type and the isomorphism types of their centralisers in $\mathrm{SU}_{3}\left(\mathbb{F}_{q^{2}}, \mathbb{F}_{q}\right)$ are summarised in Tables 7.3 and 7.4 . After recalling some preliminary tools to investigate the adjoint orbits, we discuss the eight different types.

C.1. Our computations are based on classical results of Ennola and on the Cayley map, which allows us to translate between conjugacy classes in $G:=\mathrm{GU}_{3}\left(\mathbb{F}_{q^{2}}, \mathbb{F}_{q}\right)$ and adjoint orbits in $\mathfrak{g}:=\mathfrak{g u}_{3}\left(\mathbb{F}_{q^{2}}, \mathbb{F}_{q}\right)$. We refer to [52, II.10 and VI.2] for basic properties of the Cayley map. The group $G$ and the Lie algebra $\mathfrak{g}$ are naturally embedded in the associative $\mathbb{F}_{q^{-}}$-algebra $\operatorname{Mat}_{3}\left(\mathbb{F}_{q^{2}}\right)$ with $\mathbb{F}_{q^{2}} \mid \mathbb{F}_{q^{-}}$-involution $\circ$ given by $x^{\circ}=\bar{x}^{\mathrm{t}}$, i.e. one obtains $x^{\circ}$ by applying the non-trivial Galois automorphism of $\mathbb{F}_{q^{2}} \mid \mathbb{F}_{q}$ to each entry of $x$ and then taking the transpose. For any subset $M$ of $\operatorname{Mat}_{3}\left(\mathbb{F}_{q^{2}}\right)$ let $M_{\text {gen }}$ denote the set of elements of $M$ which do not have -1 as an eigenvalue. The Cayley maps Cay : $G_{\text {gen }} \rightarrow \mathfrak{g}_{\text {gen }}$ and cay : $\mathfrak{g}_{\text {gen }} \rightarrow G_{\text {gen }}$, which are each defined by the mapping rule (7.10), are easily seen to be mutual inverses of each other, and they are compatible with conjugation by elements of $G$. 
Put $u(q):=\left|\mathrm{GU}_{3}\left(\mathbb{F}_{q^{2}}, \mathbb{F}_{q}\right)\right|=q^{3}(q+1)\left(q^{2}-1\right)\left(q^{3}+1\right)$. According to [17, two elements $X, Y \in \mathrm{GU}_{3}\left(\mathbb{F}_{q^{2}}, \mathbb{F}_{q}\right)$ are conjugate in $\mathrm{GU}_{3}\left(\mathbb{F}_{q^{2}}, \mathbb{F}_{q}\right)$ if and only if they are similar, i.e. conjugate in $\mathrm{GL}_{3}\left(\mathbb{F}_{q^{2}}\right)$. Moreover, the size of the conjugacy class in $\mathrm{GU}_{3}\left(\mathbb{F}_{q^{2}}, \mathbb{F}_{q}\right)$ consisting of matrices which are similar to a given $X \in \mathrm{GL}_{3}\left(\mathbb{F}_{q^{2}}\right)$ is $\gamma_{G}(X) u(q) c(X)^{-1}$, where

$$
\begin{aligned}
\gamma_{G}(X) & :=\mid\left\{\Gamma \in \operatorname{Mat}_{3}\left(\mathbb{F}_{q^{2}}\right) \mid \Gamma^{\circ}=\Gamma \text { non-singular, } X^{\circ} \Gamma X=\Gamma\right\} \mid, \\
c(X) & :=\left|\mathrm{C}_{\mathrm{GL}_{3}\left(\mathbb{F}_{q^{2}}\right)}(X)\right| .
\end{aligned}
$$

Suppose that $x \in \mathfrak{g}$ does not have -1 as an eigenvalue and put $X:=\operatorname{cay}(x)$. A short computation shows that for all $\Gamma \in \operatorname{Mat}_{3}\left(\mathbb{F}_{q^{2}}\right)$ with $\Gamma^{\circ}=\Gamma$ one has

$$
X^{\circ} \Gamma X=\Gamma \quad \text { if and only if } \quad x^{\circ} \Gamma+\Gamma x=0
$$

If follows that the adjoint orbit of $x$, viz.

$$
\left\{x^{g} \mid g \in G\right\}=\operatorname{Cay}\left(\left\{X^{g} \mid g \in G\right\}\right),
$$

has size $\gamma_{\mathfrak{g}}(x) u(q) c(x)^{-1}$, where

$$
\begin{aligned}
\gamma_{\mathfrak{g}}(x) & :=\mid\left\{\Gamma \in \operatorname{Mat}_{3}\left(\mathbb{F}_{q^{2}}\right) \mid \Gamma^{\circ}=\Gamma \text { non-singular, } x^{\circ} \Gamma+\Gamma x=0\right\} \mid, \\
c(x) & :=\left|\mathrm{C}_{\mathrm{GL}_{3}\left(\mathbb{F}_{q^{2}}\right)}(x)\right| .
\end{aligned}
$$

Next suppose that $x \in \mathfrak{g}$ has -1 as an eigenvalue. Since $q \geq 5$ we find $\lambda \in \mathbb{F}_{q^{2}}$ with $\operatorname{Tr}_{\mathbb{F}_{q^{2}} \mid \mathbb{F}_{q}}(\lambda)=0$ such that $x_{0}:=x+\lambda \operatorname{Id}_{3} \in \mathfrak{g}$ does not have eigenvalue -1 . Clearly, the adjoint orbits of $x$ and $x_{0}$ have the same size, $c(x)=c\left(x_{0}\right)$ and $\gamma_{\mathfrak{g}}(x)=\gamma_{\mathfrak{g}}\left(x_{0}\right)$. Hence the size of the adjoint orbit of $x$ is still given by the term $\gamma_{\mathfrak{g}}(x) u(q) c(x)^{-1}$. More generally, for any $x \in \mathfrak{g l}_{3}\left(\mathbb{F}_{q^{2}}\right)$, the same formula gives the size of the adjoint orbit in $\mathfrak{g u}_{3}\left(\mathbb{F}_{q^{2}}, \mathbb{F}_{q}\right)$ consisting of matrices which are similar to $x$.

C.2. We are now ready to discuss the eight different types. Type 0 consists of the zero matrix, which does not feature in our calculation but is shown for completeness. Its centraliser is the entire group $\mathrm{SU}_{3}\left(\mathbb{F}_{q^{2}}, \mathbb{F}_{q}\right)$.

Type 1 consists of nilpotent matrices with minimal polynomial equal to $X^{3}$ over $\mathbb{F}_{q^{2}}$. Let $x:=\left(\begin{array}{ccc}0 & 1 & \\ 0 & 1 \\ & 0\end{array}\right)$. Then the non-singular matrices $\Gamma \in \operatorname{Mat}_{3}\left(\mathbb{F}_{q^{2}}\right)$ with $\Gamma^{\circ}=\Gamma$ and $x^{\circ} \Gamma+\Gamma x=0$ are the matrices over $\mathbb{F}_{q^{2}}$ of the form

$$
\Gamma=\left(\begin{array}{ccc}
0 & 0 & \gamma \\
0 & -\gamma & \beta \\
\bar{\gamma} & \bar{\beta} & \alpha
\end{array}\right), \quad \text { where } \bar{\alpha}=\alpha, \bar{\beta}=-\beta \text { and } \bar{\gamma}=\gamma \neq 0 .
$$

This shows that $\gamma_{\mathfrak{g}}(x)=(q-1) q^{2}$. From (B.1) we gather that $c(x)=\left(q^{2}-1\right) q^{4}$ so that $\gamma_{\mathfrak{g}}(x) u(q) c(x)^{-1}=\left(q^{3}+1\right)\left(q^{2}-1\right) q$.

Put $\Gamma_{0}:=\left(\begin{array}{ccc}0 & 0 & -1 \\ 0 & 1 & 0 \\ -1 & 0 & 0\end{array}\right)$. The centraliser of a typical element of type 1 in $\mathrm{SU}_{3}\left(\mathbb{F}_{q^{2}}, \mathbb{F}_{q}\right)$ is isomorphic to

$$
\begin{aligned}
\mathrm{C}_{\mathrm{SL}_{3}\left(\mathbb{F}_{q^{2}}\right)}(x) & \cap\left\{Y \in \mathrm{GL}_{3}\left(\mathbb{F}_{q^{2}}\right) \mid Y^{\circ} \Gamma_{0} Y=\Gamma_{0}\right\} \\
= & \left\{\left(\begin{array}{lll}
a & b & c \\
0 & a & b \\
0 & 0 & a
\end{array}\right) \in \mathrm{GL}_{3}\left(\mathbb{F}_{q^{2}}\right) \mid a^{3}=a \bar{a}=1, \bar{a} b-a \bar{b}=b \bar{b}-\bar{a} c-a \bar{c}=0\right\} .
\end{aligned}
$$

The elements of this group can be conveniently parameterised in terms of $(a, a \bar{b}, a \bar{c})$ and a short computation yields that the group is isomorphic to $\left(\mu_{3}\left(\mathbb{F}_{q^{2}}\right) \cap \operatorname{ker}\left(N_{\mathbb{F}_{q^{2}} \mid \mathbb{F}_{q}}\right)\right) \times$ $\mathbb{F}_{q}^{+} \times \mathbb{F}_{q}^{+}$. Matrices of type 1 are regular. 
Type 2 consists of nilpotent matrices with minimal polynomial equal to $X^{2}$ over $\mathbb{F}_{q^{2}}$. Let $x:=\left(\begin{array}{lll}0 & 1 & 0 \\ 0 & 0 & 0 \\ 0 & 0 & 0\end{array}\right)$. Then the non-singular matrices $\Gamma \in \operatorname{Mat}_{3}\left(\mathbb{F}_{q^{2}}\right)$ with $\Gamma^{\circ}=\Gamma$ and $x^{\circ} \Gamma+\Gamma x=0$ are the matrices over $\mathbb{F}_{q^{2}}$ of the form

$$
\Gamma=\left(\begin{array}{ccc}
0 & \beta & 0 \\
\bar{\beta} & \alpha & \gamma \\
0 & \bar{\gamma} & \delta
\end{array}\right), \quad \text { where } \bar{\alpha}=\alpha, \bar{\beta}=-\beta \neq 0 \text { and } \bar{\delta}=\delta \neq 0
$$

This shows that $\gamma_{\mathfrak{g}}(x)=(q-1)^{2} q^{3}$. From (B.2) we gather that $c(x)=\left(q^{2}-1\right)^{2} q^{6}$ so that $\gamma_{\mathfrak{g}}(x) u(q) c(x)^{-1}=\left(q^{3}+1\right)(q-1)$.

Put $\Gamma_{0}:=\left(\begin{array}{ccc}0 & \sqrt{\rho} & 0 \\ -\sqrt{\rho} & 0 & 0 \\ 0 & 0 & 1\end{array}\right)$. Then the centraliser of a typical element of type 2 in $\mathrm{SU}_{3}\left(\mathbb{F}_{q^{2}}, \mathbb{F}_{q}\right)$ is isomorphic to

$$
\begin{aligned}
& \mathrm{C}_{\mathrm{SL}_{3}\left(\mathbb{F}_{q^{2}}\right)}(x) \cap\left\{Y \in \mathrm{GL}_{3}\left(\mathbb{F}_{q^{2}}\right) \mid Y^{\circ} \Gamma_{0} Y=\Gamma_{0}\right\} \\
& =\left\{\left(\begin{array}{lll}
a & b & c \\
0 & a & 0 \\
0 & d & e
\end{array}\right) \in \mathrm{GL}_{3}\left(\mathbb{F}_{q^{2}}\right) \mid a^{2} e=a \bar{a}=1, a \bar{b} \sqrt{\rho}-\bar{a} b \sqrt{\rho}+d \bar{d}=a \bar{c} \sqrt{\rho}+d \bar{e}=0\right\} .
\end{aligned}
$$

The elements of this group can be parameterised in terms of $(a, a \bar{b} \sqrt{\rho}, d \bar{e}, a \bar{c} \sqrt{\rho})$ and a short computation yields that the group is isomorphic to $\operatorname{ker}\left(N_{\mathbb{F}_{q^{2}} \mid \mathbb{F}_{q}}\right) \ltimes \mathcal{H}\left(\mathbb{F}_{q}\right)$. Indeed, conjugation by the monomial matrix $\left(\begin{array}{ccc}\sqrt{\rho} & 0 & 0 \\ 0 & 0 & 1 \\ 0 & 1 & 0\end{array}\right)^{-1}$ maps the group described in (C.2) isomorphically onto

$$
\left\{\left(\begin{array}{ccc}
a & 0 & 0 \\
0 & a^{-2} & 0 \\
0 & 0 & a
\end{array}\right) \in \mathrm{GL}_{3}\left(\mathbb{F}_{q^{2}}\right) \mid a \bar{a}=1\right\} \ltimes\left\{\left(\begin{array}{ccc}
1 & \bar{d} & b_{0} \\
0 & 1 & d \\
0 & 0 & 1
\end{array}\right) \in \mathrm{GL}_{3}\left(\mathbb{F}_{q^{2}}\right) \mid b_{0}+\overline{b_{0}}=d \bar{d}\right\} .
$$

The normal factor in this semi-direct product is seen to be isomorphic to the Heisenberg group $\mathcal{H}\left(\mathbb{F}_{q}\right)$ by 'formally setting $\sqrt{\rho}=1$ ', i.e. via the map

$$
\left(\begin{array}{ccc}
1 & \bar{d} & b_{0} \\
0 & 1 & d \\
0 & 0 & 1
\end{array}\right) \mapsto\left(\begin{array}{ccc}
1 & \varphi(\bar{d}) & \varphi\left(b_{0}\right) \\
0 & 1 & \varphi(d) \\
0 & 0 & 1
\end{array}\right), \quad \text { where } \varphi(z):=(z+\bar{z}) / 2+(z-\bar{z}) /(2 \sqrt{\rho}) .
$$

Matrices of type 2 are irregular.

Type 3 consists of semisimple matrices with eigenvalues $\lambda \in \mathbb{F}_{q^{2}} \backslash\{0\}$ of multiplicity 2 and $\mu:=-2 \lambda$ of multiplicity 1 . The condition $\bar{\lambda}=-\lambda$ implies that $\lambda \in \mathbb{F}_{q} \sqrt{\rho} \backslash\{0\}$ so that there are $q-1$ choices for $\lambda$ and $q-1$ corresponding orbits. The minimal polynomial of such elements over $\mathbb{F}_{q^{2}}$ is equal to $(X-\lambda)(X-\mu)$.

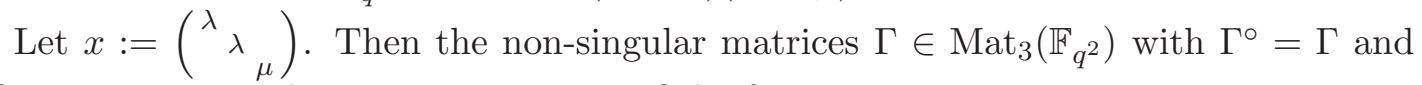
$x^{\circ} \Gamma+\Gamma x=0$ are the matrices over $\mathbb{F}_{q^{2}}$ of the form

$$
\Gamma=\left(\begin{array}{ccc}
\alpha & \beta & 0 \\
\bar{\beta} & \gamma & 0 \\
0 & 0 & \delta
\end{array}\right), \quad \text { where } \bar{\alpha}=\alpha, \bar{\gamma}=\gamma, \alpha \gamma-\beta \bar{\beta} \neq 0 \text { and } \bar{\delta}=\delta \neq 0
$$

This shows that $\gamma_{\mathfrak{g}}(x)=\left(q^{2}+1\right)(q-1) q$. From (B.3) we gather that $c(x)=\left(q^{4}-1\right)\left(q^{2}-\right.$ $1)^{2} q^{2}$ so that the size of each orbit is $\gamma_{\mathfrak{g}}(x) u(q) c(x)^{-1}=\left(q^{2}-q+1\right) q^{2}$.

Taking $\Gamma_{0}:=\mathrm{Id}_{3}$, the centraliser of the typical element $x$ of type 3 in $\mathrm{SU}_{3}\left(\mathbb{F}_{q^{2}}, \mathbb{F}_{q}\right)$ is

$$
\begin{aligned}
& \mathrm{C}_{\mathrm{SU}_{3}\left(\mathbb{F}_{q^{2}}, \mathbb{F}_{q}\right)}(x) \\
& \quad=\left\{\left(\begin{array}{lll}
a & b & 0 \\
c & d & 0 \\
0 & 0 & e
\end{array}\right) \in \mathrm{GL}_{3}\left(\mathbb{F}_{q^{2}}\right) \mid a \bar{a}+c \bar{c}=b \bar{b}+d \bar{d}=(a d-b c) e=1, a \bar{b}+c \bar{d}=0\right\} .
\end{aligned}
$$

Inspection shows that this group is isomorphic to $\mathrm{GU}_{2}\left(\mathbb{F}_{q^{2}}, \mathbb{F}_{q}\right)$, and matrices of type 3 are irregular. 
Types $4 \mathrm{a}, 4 \mathrm{~b}$ and $4 \mathrm{c}$ classify semisimple matrices with distinct non-zero eigenvalues $\lambda, \mu, \nu:=-\lambda-\mu$ in an extension of $\mathbb{F}_{q^{2}}$. The minimal polynomial of such elements over $\mathbb{F}_{q^{2}}$ is equal to $(X-\lambda)(X-\mu)(X-\nu)$. The condition $\{\bar{\lambda}, \bar{\mu},-\bar{\lambda}-\bar{\mu}\}=\{-\lambda,-\mu, \lambda+\mu\}$ allows for three possibilities.

Type 4a consists of those semisimple matrices where $\bar{\lambda}=-\lambda$ and $\bar{\mu}=-\bar{\mu}$ so that, in particular, $\lambda, \mu \in \mathbb{F}_{q^{2}} \backslash\{0\}$. The condition that $\lambda, \mu,-\lambda-\mu$ are distinct means that $\mu \notin\{\lambda,-2 \lambda,-\lambda / 2\}$. A short computation shows that there are $(q-1)(q-2) / 6$ possibilities for $\{\lambda, \mu, \nu\}$ and hence a corresponding number of orbits.

Let $x:=\left(\begin{array}{cc}\lambda & \\ & \\ & \nu\end{array}\right)$. Then the non-singular matrices $\Gamma \in \operatorname{Mat}_{3}\left(\mathbb{F}_{q^{2}}\right)$ with $\Gamma^{\circ}=\Gamma$ and $x^{\circ} \Gamma+\Gamma x=0$ are the matrices over $\mathbb{F}_{q^{2}}$ of the form

$$
\Gamma=\left(\begin{array}{ccc}
\alpha & 0 & 0 \\
0 & \beta & 0 \\
0 & 0 & \gamma
\end{array}\right), \quad \text { where } \bar{\alpha}=\alpha \neq 0, \bar{\beta}=\beta \neq 0 \text { and } \bar{\gamma}=\gamma \neq 0
$$

This shows that $\gamma_{\mathfrak{g}}(x)=(q-1)^{3}$. From (B.4) we gather that $c(x)=\left(q^{2}-1\right)^{3}$ so that the size of each orbit is $\gamma_{\mathfrak{g}}(x) u(q) c(x)^{-1}=\left(q^{2}-q+1\right)(q-1) q^{3}$.

Taking $\Gamma_{0}:=\mathrm{Id}_{3}$, the centraliser of the typical element $x$ of type $4 a$ in $\mathrm{SU}_{3}\left(\mathbb{F}_{q^{2}}, \mathbb{F}_{q}\right)$ is

$$
\mathrm{C}_{\mathrm{SU}_{3}\left(\mathbb{F}_{q^{2}}, \mathbb{F}_{q}\right)}(x)=\left\{\left(\begin{array}{lll}
a & 0 & 0 \\
0 & b & 0 \\
0 & 0 & c
\end{array}\right) \in \mathrm{GL}_{3}\left(\mathbb{F}_{q^{2}}\right) \mid a \bar{a}=b \bar{b}=a b c=1\right\} .
$$

Inspection shows that this group is isomorphic to $\operatorname{ker}\left(N_{\mathbb{F}_{q^{2}} \mid \mathbb{F}_{q}}\right) \times \operatorname{ker}\left(N_{\mathbb{F}_{q^{2}} \mid \mathbb{F}_{q}}\right)$, and matrices of type $4 \mathrm{a}$ are regular.

Type $4 \mathrm{~b}$ consists of those semisimple matrices where $\bar{\lambda}=-\mu$ and $\bar{\mu}=-\lambda$ so that, in particular, $\lambda, \mu \in \mathbb{F}_{q^{2}} \backslash\{0\}$. The condition that $\lambda, \mu=-\bar{\lambda}, \nu=\bar{\lambda}-\lambda$ are distinct means that $\lambda \notin\{-\bar{\lambda}, 2 \bar{\lambda}, \bar{\lambda} / 2\}$. Thus there are $q(q-1) / 2$ possibilities for $\{\lambda, \mu, \nu\}$ and a corresponding number of orbits.

Let $x:=\left(\begin{array}{c}\lambda \\ { }^{\mu}\end{array}\right)$. Then the non-singular matrices $\Gamma \in \operatorname{Mat}_{3}\left(\mathbb{F}_{q^{2}}\right)$ with $\Gamma^{\circ}=\Gamma$ and $x^{\circ} \Gamma+\Gamma x=0$ are the matrices over $\mathbb{F}_{q^{2}}$ of the form

$$
\Gamma=\left(\begin{array}{ccc}
0 & \alpha & 0 \\
\bar{\alpha} & 0 & 0 \\
0 & 0 & \beta
\end{array}\right), \quad \text { where } \alpha \neq 0 \text { and } \bar{\beta}=\beta \neq 0 .
$$

This shows that $\gamma_{\mathfrak{g}}(x)=(q+1)(q-1)^{2}$. From (B.4) we gather that $c(x)=\left(q^{2}-1\right)^{3}$ so that the size of each orbit is $\gamma_{\mathfrak{g}}(x) u(q) c(x)^{-1}=\left(q^{3}+1\right) q^{3}$.

Put $\Gamma_{0}:=\left(\begin{array}{lll}0 & 1 & 0 \\ 1 & 0 & 0 \\ 0 & 0 & 1\end{array}\right)$. Then the centraliser of a typical element of type $4 \mathrm{~b}$ in $\mathrm{SU}_{3}\left(\mathbb{F}_{q^{2}}, \mathbb{F}_{q}\right)$ is isomorphic to

$$
\begin{aligned}
\mathrm{C}_{\mathrm{SL}_{3}\left(\mathbb{F}_{q^{2}}\right)}(x) \cap\left\{Y \in \mathrm{GL}_{3}\left(\mathbb{F}_{q^{2}}\right) \mid Y^{\circ} \Gamma_{0} Y\right. & \left.=\Gamma_{0}\right\} \\
= & \left\{\left(\begin{array}{lll}
a & 0 & 0 \\
0 & b & 0 \\
0 & 0 & c
\end{array}\right) \in \mathrm{GL}_{3}\left(\mathbb{F}_{q^{2}}\right) \mid a b c=a \bar{b}=1\right\} .
\end{aligned}
$$

The elements of this group can be conveniently parameterised in terms of $a$ and the group is isomorphic to $\mathbb{F}_{q^{2}}^{*}$. Matrices of type $4 \mathrm{~b}$ are regular.

Type 4c consists of those semisimple matrices where $\bar{\lambda}=-\mu, \bar{\mu}=-\nu$ and, consequently $\bar{\nu}=-\lambda$. Then $\nu=-\lambda-\mu$ implies that $\overline{\bar{\lambda}}-\bar{\lambda}+\lambda=0$. A short computation yields that the relevant values for $\lambda$ are $\lambda=\lambda_{0} \sqrt{\rho}$, where $\lambda_{0} \in F_{q^{3}} \backslash\{0\}$ with $\operatorname{Tr}_{\mathbb{F}_{q^{3}} \mid \mathbb{F}_{q}}\left(\lambda_{0}\right)=0$. In particular, it follows that $\mathbb{F}_{q}(\lambda)=\mathbb{F}_{q^{6}}$. There are $\left(q^{2}-1\right) / 3$ possibilities for $\{\lambda, \mu, \nu\}$ and a corresponding number of orbits. 
Let $x$ be an element of type 4c. Then the non-singular matrices $\Gamma \in \operatorname{Mat}_{3}\left(\mathbb{F}_{q^{2}}\right)$ with $\Gamma^{\circ}=\Gamma$ and $x^{\circ} \Gamma+\Gamma x=0$ are in one-to-one correspondence to non-zero elements of $\mathfrak{g u}_{1}\left(\mathbb{F}_{q^{6}}, \mathbb{F}_{q^{3}}\right)$. This shows that $\gamma_{\mathfrak{g}}(x)=q^{3}-1$. The centraliser of $x$ in $\mathrm{GL}_{3}\left(\mathbb{F}_{q^{2}}\right)$ is isomorphic to $\mathbb{F}_{q^{6}}^{*}$ so that $c(x)=q^{6}-1$. Therefore the size of each orbit is $\gamma_{\mathfrak{g}}(x) u(q) c(x)^{-1}=\left(q^{2}-1\right)(q+1) q^{3}$.

The centraliser of a typical element of type $4 \mathrm{c}$ in $\mathrm{GU}_{3}\left(\mathbb{F}_{q^{2}}, \mathbb{F}_{q}\right)$ is isomorphic to $\mathrm{GU}_{1}\left(\mathbb{F}_{q^{6}}, \mathbb{F}_{q^{3}}\right) \cong \operatorname{ker}\left(N_{\mathbb{F}_{q^{6}} \mid \mathbb{F}_{q^{3}}}\right)$. The centraliser of such an element in $\mathrm{SU}_{3}\left(\mathbb{F}_{q^{2}}, \mathbb{F}_{q}\right)$ is isomorphic to $\operatorname{ker}\left(N_{\mathbb{F}_{q^{6}} \mid \mathbb{F}_{q^{3}}}\right) \cap \operatorname{ker}\left(N_{\mathbb{F}_{q^{6}} \mid \mathbb{F}_{q^{2}}}\right)$. Matrices of type $4 \mathrm{c}$ are regular.

Type 5 consists of semisimple matrices with eigenvalues $\lambda \in \mathbb{F}_{q^{2}} \backslash\{0\}$ of multiplicity 2 and $\mu:=-2 \lambda$ of multiplicity 1 . The condition $\bar{\lambda}=-\lambda$ implies that $\lambda \in \mathbb{F}_{q \sqrt{\rho} \backslash\{0\} \text { so }}$ that there are $q-1$ choices for $\lambda$ and $q-1$ corresponding orbits. The minimal polynomial of such elements over $\mathbb{F}_{q^{2}}$ is equal to $(X-\lambda)^{2}(X-\mu)$.

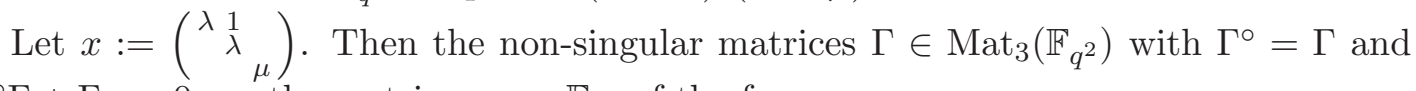
$x^{\circ} \Gamma+\Gamma x=0$ are the matrices over $\mathbb{F}_{q^{2}}$ of the form

$$
\Gamma=\left(\begin{array}{ccc}
0 & \beta & 0 \\
\bar{\beta} & \alpha & 0 \\
0 & 0 & \gamma
\end{array}\right), \quad \text { where } \bar{\alpha}=\alpha, \bar{\beta}=-\beta \neq 0 \text { and } \bar{\gamma}=\gamma \neq 0 .
$$

This shows that $\gamma_{\mathfrak{g}}(x)=(q-1)^{2} q$. From (B.5) we gather that $c(x)=\left(q^{2}-1\right)^{2} q^{2}$ so that the size of each orbit is $\gamma_{\mathfrak{g}}(x) u(q) c(x)^{-1}=\left(q^{3}+1\right)(q-1) q^{2}$.

Put $\Gamma_{0}:=\left(\begin{array}{ccc}0 & \sqrt{\rho} & 0 \\ -\sqrt{\rho} & 0 & 0 \\ 0 & 0 & 1\end{array}\right)$. Then the centraliser of a typical element of type 5 in $\mathrm{SU}_{3}\left(\mathbb{F}_{q^{2}}, \mathbb{F}_{q}\right)$ is isomorphic to

$$
\begin{aligned}
\mathrm{C}_{\mathrm{SL}_{3}\left(\mathbb{F}_{q^{2}}\right)}(x) \cap\left\{Y \in \mathrm{GL}_{3}\left(\mathbb{F}_{q^{2}}\right) \mid Y^{\circ} \Gamma_{0} Y=\Gamma_{0}\right\} \\
=\left\{\left(\begin{array}{lll}
a & b & 0 \\
0 & a & 0 \\
0 & 0 & c
\end{array}\right) \in \mathrm{GL}_{3}\left(\mathbb{F}_{q^{2}}\right) \mid a^{2} c=a \bar{a}=1, a \bar{b}-\bar{a} b=0\right\} .
\end{aligned}
$$

The elements of this group can be conveniently parameterised in terms of $(a, a \bar{b})$ and a short computation yields that the group is isomorphic to $\operatorname{ker}\left(N_{\mathbb{F}_{q^{2}} \mid \mathbb{F}_{q}}\right) \ltimes \mathbb{F}_{q}^{+}$. Matrices of type 5 are regular.

Acknowledgements. The authors would like to thank Alexander Lubotzky as well as the following institutions: the Batsheva de Rothschild Fund for the Advancement of Science, the EPSRC, the Mathematisches Forschungsinstitut Oberwolfach, the National Science Foundation and the Nuffield Foundation.

\section{REFERENCES}

[1] N. Avni, Arithmetic groups have rational representation growth, arXiv:math.GR/0803.1331v1 (2008).

[2] N. Avni, B. Klopsch, U. Onn, C. Voll, On representation zeta functions of groups and a conjecture of Larsen-Lubotzky, C. R. Math. Acad. Sci. Paris, Ser. I 348 (2010), 363-367.

[3] N. Avni, B. Klopsch, U. Onn, C. Voll, Representation zeta functions for $\mathrm{SL}_{3}$, preprint.

[4] N. Avni, B. Klopsch, U. Onn, C. Voll, Representation zeta functions of some compact p-adic analytic groups, preprint.

[5] H. Bass, A. Lubotzky, A.R. Magid, S. Mozes, The proalgebraic completion of rigid groups, Proceedings of the Conference on Geometric and Combinatorial Group Theory, Part II (Haifa, 2000), Geom. Dedicata 95 (2002), 19-58.

[6] W. Borho, Über Schichten halbeinfacher Lie-Algebren, Invent. Math. 65 (1981/82), 283-317.

[7] J. Brill, On the minors of a skew-symmetrical determinant, Proc. London Math. Soc. 34 (1904), 103-111. 
[8] A. Broer, Decomposition varieties in semisimple Lie algebras, Can. J. Math. 50 (1998), 929-971.

[9] C.J. Bushnell and P.C. Kutzko, The admissible dual of GL $(N)$ via compact open subgroups, Annals of Mathematics Studies 129, Princeton University Press, Princeton, NJ, 1993.

[10] J.W.S. Cassels and A. Fröhlich (editors), Algebraic number theory, Proceedings of an instructional conference organized by the London Mathematical Society, 2nd revised edition, London Mathematical Society, London, 2010.

[11] J. Denef, On the degree of Igusa's local zeta function, Amer. J. Math. 109 (1987), 991-1008.

[12] J. Denef, Report on Igusa's local zeta function, Séminaire Bourbaki, Vol. 1990/91.

[13] J. Denef, D. Meuser, A functional equation of Igusa's local zeta function, Amer. J. Math. 113 (1991), 1135-1152.

[14] J. Dixon, M. du Sautoy, A. Mann, D. Segal, Analytic pro- $p$ groups, 2nd ed., Cambridge University Press, Cambridge, 1999.

[15] M. du Sautoy, F. Grunewald, Analytic properties of zeta functions and subgroup growth, Ann. of Math. 152 (2000), 793-833.

[16] M. du Sautoy, F. Grunewald, Zeta functions of groups and rings, in: International Congress of Mathematicians, Vol. II, 131-149, Eur. Math. Soc., Zürich, 2006.

[17] V. Ennola, On the conjugacy classes of the finite unitary groups, Ann. Acad. Sci. Fenn. Ser. A I 313 (1962) 13pp.

[18] J. González-Sánchez, On p-saturable groups, J. Algebra 315 (2007), 809-823.

[19] J. González-Sánchez, Kirillov's orbit method for p-groups and pro-p groups, Comm. Algebra 37 (2009), 4476-4488.

[20] J. González-Sánchez, B. Klopsch, Analytic pro-p groups of small dimensions, J. Group Theory 12 (2009), 711-734.

[21] M.J. Greenberg, Schemata over local rings, Ann. Math. (2) 73 (1961), 624-648.

[22] B.H. Gross, G. Nebe, Globally maximal arithmetic groups, J. Algebra 272 (2004), 625-642.

[23] P. Heymans, Pfaffians and skew-symmetric matrices, Proc. London Math. Soc. 19 (1969), 730-768.

[24] H. Hironaka, Resolution of singularities of an algebraic variety over a finite field of characteristic zero. I, II, Ann. of Math.(2) 79 (1964), 205-326.

[25] R.E. Howe, On representations of discrete, finitely generated, torsion-free, nilpotent groups, Pacific J. Math. 73 (1977), 281-305.

[26] R.E. Howe, Kirillov theory for compact p-adic groups, Pacific J. Math. 73 (1977), 365-381.

[27] E. Hrushovski, B. Martin, Zeta functions from definable equivalence relations, arXiv:math/070111 (2007).

[28] B. Huppert, Character theory of finite groups, de Gruyter Expositions in Mathematics 25, Walter de Gruyter \& Co., Berlin, 1998.

[29] J. Igusa, An introduction to the theory of local zeta functions, AMS/IP Studies in Advanced Mathematics 14, American Mathematical Society, Providence, RI, International Press, Cambridge, MA, 2000.

[30] I.M. Isaacs, Counting characters of upper triangular groups, J. Algebra 315 (2007), 698-719.

[31] A. Jaikin-Zapirain, Zeta function of representations of compact p-adic analytic groups, J. Amer. Math. Soc. 19 (2006), 91-118.

[32] N. Kawanaka, Generalized Gel'fand-Graev representations and Ennola duality, in: Algebraic groups and related topics (Kyoto/Nagoya, 1983), 175-206, Adv. Stud. Pure Math. 6, North-Holland, Amsterdam, 1985.

[33] H.D. Kloosterman, The behaviour of general theta functions under the modular group and the characters of binary modular congruence groups. I,II., Ann. of Math. 47 (1946), 317-447.

[34] B. Klopsch, On the Lie theory of p-adic analytic groups, Math. Z. 249 (2005), 713-730.

[35] M. Larsen, A. Lubotzky, Representation growth of linear groups, J. Eur. Math. Soc. (JEMS) 10 (2008), 351-390.

[36] M. Lazard, Groupes analytiques p-adiques, Publ. Math. IHÉS 26 (1965), 389-603.

[37] M. Liebeck, A. Shalev, Character degrees and random walks in finite groups of Lie type, Proc. London Math. Soc. 90 (2005), 61-86.

[38] A. Lubotzky, B. Martin, Polynomial representation growth and the congruence subgroup problem, Israel J. Math. 144 (2004), 293-316.

[39] A. Lubotzky, D. Segal, Subgroup growth, Progress in Mathematics 212, Birkhäuser Verlag, Basel, 2003. 
[40] F. Lübeck, Character Degrees and their Multiplicities for some Groups of Lie Type of Rank $<9$, online data, http://www.math.rwth-aachen.de/ Frank.Luebeck/chev/DegMult/index.html.

[41] D. Meuser, The meromorphic continuation of a zeta function of Weil and Igusa type, Invent. Math. 85 (1986), 493-514.

[42] A. Moretó, Characters of p-groups and Sylow p-subgroups, in: Groups St. Andrews 2001 in Oxford, Vol. II, 412-421, London Math. Soc. Lecture Note Ser. 305, Cambridge Univ. Press, Cambridge, 2003.

[43] V. Platonov, A. Rapinchuk, Algebraic groups and number theory, Pure and Applied Mathematics 139, Academic Press, Inc., Boston, 1994.

[44] I. Reiner, Maximal orders, London Mathematical Society Monographs 5, Academic Press, LondonNew York, 1975.

[45] W. Scharlau, Quadratic and Hermitian forms, Grundlehren der Mathematischen Wissenschaften 270, Springer-Verlag, Berlin, 1985.

[46] J.-P. Serre, Le problème des groupes de congruence pour $\mathrm{SL}_{2}$, Ann. of Math. (2) 92 (1970), 489-527.

[47] J.-P. Serre, Local fields, Graduate Texts in Mathematics 67, Springer-Verlag, New York-Berlin, 1979.

[48] R. Stanley, Combinatorics and Commutative Algebra, Second Edition, Progress in Mathematics 41, Birkhäuser, 1996.

[49] R. Stanley, Enumerative Combinatorics, Volume 1, Cambridge Studies in Advanced Mathematics 49, Cambridge University Press, 1997.

[50] W. Veys, W.A. Zúñiga-Galindo, Zeta functions for analytic mappings, log-principalization of ideals, and Newton polyhedra, Trans. Amer. Math. Soc. 360 (2008), 2205-2227.

[51] C. Voll, Functional equations for zeta functions of groups and rings, Ann. of Math. (2) 172 (2010), 1181-1218.

[52] H. Weyl, The classical groups, Their invariants and representations, Princeton University Press, Princeton, NJ, 1997.

Department of Mathematics, Harvard University, One Oxford Street, Cambridge Ma 02138, USA

E-mail address: avni.nir@gmail.com

Department of Mathematics, Royal Holloway, University of London, Egham TW20 0EX, United KINGDOM

E-mail address: Benjamin.Klopsch@rhul.ac.uk

Department of Mathematics, Ben Gurion University of the Negev, Beer-Sheva 84105 ISRAEL

E-mail address: urionn@math.bgu.ac.il

School of Mathematics, University of Southampton, University Road, Southampton SO17 1BJ, United Kingdom

E-mail address: C.Voll.98@cantab.net 